\title{
Type specimens of Birds in the Museo Regionale di Scienze Naturali (Torino, Italy)
}

\author{
Giorgio Aimassi ${ }^{1,}$, , Claudio Pulcher $^{2}$ \& Luca Ghiraldi ${ }^{3}$ \\ ${ }^{1}$ Museo Civico «F. Eusebio», Via Paruzza 1, I-12051 Alba, Italy; email: gaimass@tin.it \\ ${ }^{2}$ Gruppo Piemontese Studi Ornitologici-Museo Civico di Storia Naturale, Via S. Francesco di Sales 188, \\ I-10022 Carmagnola, Italy \\ ${ }^{3}$ Museo Regionale di Scienze Naturali, Via Giolitti 36, I-10123 Torino, Italy \\ * corresponding author
}

Aimassi G., Pulcher C. \& Ghiraldi L., 2020:Type specimens of Birds in the Museo Regionale di Scienze Naturali (Torino, Italy). - Journal of the National Museum (Prague), Natural History Series 189: 65-154.

\begin{abstract}
Since the 1990s, the Museo Regionale di Scienze Naturali di Torino houses the ornithological collection formerly belonging to the Zoological Museum of the University of Turin (MZUT). This collection includes about 20,500 specimens, mostly dating from the second half of the nineteenth century or early twentieth. The high number of type-specimens gives it great historical and scientific significance. The types have been described mainly by Tommaso Salvadori (171 taxa, 282 specimens) and, to a lesser extent, by other Italian authors such as Enrico Festa, Filippo de Filippi, Orazio Antinori, Enrico H. Giglioli or by foreign authors as John Gould, Eduard Rüppell, Coenraad Jacob Temminck, Philip L. Sclater, Robert Swinhoe.
\end{abstract}

Keywords: type-specimens, birds, Turin museum, Tommaso Salvadori, Italian explorers.

Received: December 16, 2019 | Accepted: November 22, 2020 | Published on-line: December 28, 2020

\section{Historical notes on the Museum}

Most of the historical information on the ornithological collection of the Zoological Museum of the Turin University (MZUT), now hosted at the Museo Regionale di Scienze Naturali (MRSN, Torino, Italy), can be found in the works of Salvadori (1915), Pierantoni (1925), Tortonese (1957), Passerin d'Entrèves et Sella (1985), Elter (1986), Passerin d'Entrèves et al. (1986). Some more updated information is summarized in Roselaar (2003: 293).

The collection began in 1811 when Franco Andrea Bonelli (1784-1830) was appointed professor of zoology and director of the museum at the University of Turin. The designation of Bonelli was supported by Georges Cuvier, who a year earlier had invited him to Paris for a period of specialization at the Muséum d'Histoire naturelle.

No descriptions of new species were published by Bonelli, who limited himself in gathering his observations in a manuscript catalogue and forwarded many specimens to colleagues all over Europe. To reciprocate, in 1819 Vieillot dedicated the new species Sylvia bonelli to the scientist of Turin and, a few years later, Temminck described Falco bonelli. 
Furthermore, Temminck sent to Bonelli a number of interesting specimens, many of which from Java and described by Temminck himself.

During his short life Bonelli had the opportunity to travel to Paris and London, bringing home many skins. Among the birds he acquired from W. Bullock's collection (59 specimens still in museum database) was also present the specimen that Salvadori (1908a) described as Fregetta melanoleuca.

Bonelli's work was continued by Giuseppe Gené (1800-1847), who enlarged the ornithological collection with material from his journeys to Sardinia and described some European species. He also sent several specimens to other ornithologists. As a reward, some new species, such as Larus genei Breme, were dedicated to him. As indicated below, when he was director of the museum, Gené received a number of important specimens from Abyssinia (Ethiopia), mostly donated by E. Rüppell and he had a valuable collection of Australian birds from J. Gould. Furthermore, in the MZUT collection there is a mounted specimen of the extinct species Athene albifacies, purchased in Milano by Gené (Ghiraldi et Aimassi 2019).

Filippo De Filippi (1814-1867) succeeded Gené as director in 1848. Naturalist and renowned politician, he collected specimens in his travel to Persia (De Filippi 1865) and in the first Italian round-the-world scientific voyage with the warship Magenta in 1865-1867, until his death in Hong Kong (Giglioli 1876).

During the direction of De Filippi and that of his successors Michele Lessona and Lorenzo Camerano, the most famous ornithologist who worked at the Zoological Museum of Turin was undoubtedly Tommaso Salvadori (1835-1923). He was museum curator and vice-director for more than 50 years, from 1865 to 1921. His work was impressive, and he managed to raise the number of bird specimens from about 5000 to over 20,000, giving the Museum an international reputation (in the early twentieth century, the collection included 13,036 specimens exhibited to the public and about 8,000 skins).

After the Salvadori retirement the ornithological collection was almost abandoned, and the same happened to all vertebrates. The collection was severely damaged by bombs during World War II and the majority of mounted specimens were dismounted for lack of space.

Many years later, the most important work was done by the curator Orsetta Elter, who finally published a catalogue of the entire collection; this is a non-critical work and "its sole aim is to provide specialists with a list of the ornithological material kept in the Museum of Zoology of the Turin University" (Elter 1986). Despite the author's statement, this has been a groundwork for any further study on the collection.

At the end of the twentieth century Claudio Pulcher undertook an important work of study and revision of the entire MZUT collection, with the collaboration of the taxidermist Franco Ferrero. This work has proved indispensable to the preparation of this catalogue.

\section{The Italian explorers}

Many scientific expeditions of Italian explorers and naturalists have recently been described by Mazzotti (2011) and by Maretti et al. (2013), while some important reports were published by the protagonists themselves (Beccari 1902, De Filippi 1865, Giglioli 1876).

In the years 1865-1894 some Italian researchers visited Papua and the surrounding areas, sending home a series of specimens of enormous value. Their contributions to the MZUT collection are significative: Odoardo Beccari sent 369 specimens still in the collection, 34 of which are type-specimens (22 taxa); Luigi Maria D'Albertis - 371 specimens, 28 types (22 taxa); Giacomo Doria - 157 specimens, 3 types ( 3 taxa); Leonardo Fea - 102 specimens, 4 types ( 3 taxa); Elio Modigliani - 46 specimens, 19 types (14 taxa).

Such expeditions were mainly supported by Marquis G. Doria, the founder of the Natural History Museum of Genova. Doria sent all the birds to Salvadori for study, allowing in exchange to Turin Museum a remarkable number of specimens. In the Genova museum are still present the types of 294 taxa described by Salvadori (Arbocco et al. 1979, 1986), 60 of which 
have type-specimens also in the MZUT collection. A detailed account of the explorations of Italian naturalists in New Guinea was written by Doria (1878).

During the Magenta expedition, when Professor De Filippi died at Hong Kong in 1867, twenty-two-year-old Enrico H. Giglioli took command of the scientific team. Giglioli and Salvadori described many new species collected in the course of this scientific enterprise: among them we can recall the Procellariidae Pterodroma defilippiana, P. magentae and P. arminjoniana, the last after V. Arminjon, captain of the Magenta (Mearns et Mearns 1998). The ornithological collection of Torino still includes 1,148 specimens (among which there are 16 types) coming from this expedition.

The interest of Italian explorers such as Orazio Antinori, Odoardo Beccari, Vincenzo Ragazzi, Eugenio Ruspoli, was also aimed at eastern Africa, where they did numerous expeditions. Again, specimens were studied mainly by Salvadori, but many collections were dispersed among different institutions. On the basis of specimens still housed in the Museum of Turin, the main contributors appear Antinori (980 specimens, 48 types of 23 taxa) and Ragazzi (702 specimens, 25 types of 12 taxa)

Some Italian explorers have begun visiting the South America since the 1890s, but without entering into the Amazon basin. Thanks to them Salvadori received many interesting species mostly from Argentina, Paraguay, Bolivia and Brazil. The MZUT still holds 989 specimens (18 types of 12 taxa) collected by Alfredo Borelli. In 1895 Enrico Festa planned a big voyage from Ecuador to Amazonas, crossing the Andes, but some problems forced him to stay in Ecuador. In this and other expeditions, Festa collected 3,324 specimens of birds, including 64 types of 25 different taxa, still in the Museum of Turin.

\section{The current list of types}

In order to draw up a list of the types present in the MZUT ornithological collection, we first checked the specimens referred to as types by Salvadori (1915). The taxa with type-specimens ("types" and "cotypes") listed by Salvadori (1915) are 304, but there is some discrepancy between the index (pages 44-47) and the main text.

From the analysis of the manuscript catalogues, of the labels that accompany each specimen and of the available bibliography, it has been possible to identify some other type-specimens, whose status is discussed below.

Most of the species concerned with this paper was described in the Annali del Museo civico di Storia naturale di Genova in 1874-1894 (104 species) and in the Bollettino dei Musei di Zoologia ed Anatomia comparata della R. Università di Torino in 1894-1916 (46 species). The dating of these species presents few problems, because the dates of the Annali have been fixed by Poggi $(1996,2010)$ and all the issues of the Bollettino have the day of publication printed on their first page. However, a significant number of Salvadori's taxa was described in Atti della Società Italiana di Scienze Naturali (SISN) in 1864-1869 (22 species) and in Atti della Reale Accademia delle Scienze di Torino in 1868-1885 (20 species).

The publication dates of the Atti SISN have been recently revised by Galbreath et Aimassi (2020) but, during the research, we also had access to some separately printed papers, with their own pagination and date of publication, which can be regarded as preprints according to the International Code of Zoological Nomenclature (ICZN 1999, 2012). These preprints have allowed to suggest some changes to the publication dates adopted so far for some species described by Salvadori and by Giglioli \& Salvadori.

We also examined the issues of the Atti della Reale Accademia delle Scienze di Torino, which Dickinson (2011a) indicated as little explored and sometimes problematic, but we must admit that the dating of Salvadori's species has not yet been fully resolved.

The MZUT collection includes several specimens donated by other ornithologists, which belong to the species described by them. In many cases it was difficult to prove that these were really type specimens, particularly when in the original descriptions there was no ex- 
plicit reference to the specimens that had actually been studied by the authors. The greatest uncertainties occurred when the specimens sent to the MZUT were not provided with complete capture data.

John Gould offered to the Museum of Turin many Australian birds, probably in 18421843 (Salvadori 1915: 13) or shortly before, after returning to Europe in 1840 (Salvadori 1881a: 6, footnote 1), when also for this he was named Corresponding Member of the Reale Accademia delle Scienze di Torino. In Salvadori (1915: 13) they are all referred to as "cotypes" but it was not possible to accurately check the status of each. In uncertain cases we have limited ourselves to indicating them as "possible" syntypes.

Coenraad Jacob Temminck, the founder and first director of the Leiden Museum, in the years 1815-1830 (mainly in 1826), sent to the Museum of Turin almost 100 specimens, some of which belonging to species described by him. The latter, according to Salvadori (1915: 7-8), had mostly been collected in Java by Heinrich Kuhl and Johan Coenraad van Hasselt and should be regarded as "types or cotypes". In Salvadori's paper, however, some specimens are listed as types of species that Temminck never described: mostly they correspond to names only used in some manuscript text (MS. hereafter), such as those we can read on the original labels or on ancient catalogues.

In 1823, when F.A. Bonelli was director of the Museum, Prince Maximilian Wied zu Neuwied sent a series of duplicates of his collection and Salvadori (1915: 8) stated that they were all to be considered as "cotypes" of the species described by Wied, even in the following years. Actually, only one out of the 11 specimens donated by Wied and still existing can be considered a syntype.

Some duplicates of Eduard Rüppell's famous collection, coming from Abyssinia (Ethiopia), were sent to the MZUT in 1839 (Salvadori 1915: 10). As can be read on most of the original labels, which also bear the Rüppell Collection number, they were collected in 1832 .

Salvadori (1915) considered as type-specimens almost all the birds sent by these ornithologists, when belonging to species described by them. However, on several occasions the dates of entry into the Museum of Turin precede the description by some years, making it difficult to ascertain whether they were types or not. At least in some cases, it can be assumed that at the time of sending the specimens were already provided with labels with the MS. indication of the name that, some years later, the author would have used to describe them formally. For those birds it can be correct to consider them as syntypes, because they are part of the specimens that the author had certainly examined (ICZN 1999, Art. 72.4.1.1).

In this catalogue, 402 type specimens of the MZUT collection are listed, which correspond to: 72 holotypes, 2 lectotypes, 303 syntypes and 25 paratypes or paralectotypes. Most of them (282 specimens, 171 species, 111 still valid) was described by T. Salvadori, in some cases together with co-authors, such as E. Festa (23 species), E.H. Giglioli ( 9 species), L.M. D’Albertis (one species).

\section{Data arrangement}

All entries concerning type-specimens are arranged according to the following standard:

- name of the taxon in original spelling, Author(s) and year of publication;

- citation (abbreviated);

- valid name; the nomenclature and the arrangement of families, species and subspecies follow Dickinson et Remsen (2013) and Dickinson et Christidis (2014);

- type-status, inventory number (MZUT Av = Museum of Zoology, University of Torino, Aves), preparation, sex and age, locality, capture date, origin / provenance, way and date of acquisition, collector or former collection.

Additional information is provided whenever necessary and evidence for the type status is discussed. 
Specimens already quoted as type-specimens by Salvadori (1915) or by Elter (1986) but that in our opinion are not, are treated in the "notes" of the main text or in Appendix 1. Some types reported in the MZUT by Salvadori (1915) were already missing when Elter (1986) drew up the catalogue of the entire MZUT collection, then still housed in the University of Turin. The list is provided in Appendix 2.

\section{LIST OF TAXA WITH TYPE SPECIMENS IN THE MZUT}

\section{ANATIDAE}

Plectropterus scioanus Salvadori, 1884

Salvadori T., 1884, Ann. Mus. Civ. Genova, Serie 2a 1 (XXI): 238-239.

Now: Plectropterus gambensis gambensis (Linnaeus, 1766)

SYNTYPE; MZUT Av10313 - mounted skin; adult male; Ethiopia, Shewa, "Cialalakà" Lake (Cheleleka or Dabashi Lake), Hadda Huash', 26 November 1879; collected by O. Antinori (field number 1143);

SYNTYPE; MZUT Av10314 - mounted skin; adult male; Ethiopia, Shewa, "Cialalakà" Lake, Hadda Huash', 24 April 1879; collected by O. Antinori (field number 898);

SYNTYPE; MZUT Av10315 - mounted skin; immature male; Ethiopia, Shewa, "Cialalakà" Lake, Hadda Huash', 14 April 1879; collected by 0. Antinori (field number 871).

Notes: Salvadori (1915: 32) erroneously omits specimen Av10314 from his list of Types. According to Reichenow (1900: 136) P. scioanus = P. niger Sclater 1877, however the area of $P$. gambensis niger does not include the Shewa, where there is the type-locality of the Salvadori species (Stresemann et Amadon in Mayr et Cottrell 1979: 453; del Hoyo et Collar 2014: 134).

\section{MEGAPODIIDAE}

Talegallus fuscirostris Salvadori, 1877

Salvadori T., 1877, Ann. Mus. Civ. Genova, IX: 330-334.

Now: Talegalla fuscirostris fuscirostris Salvadori, 1877

SYNTYPE; MZUT Av9962 - mounted skin; adult male; Indonesia, Aru, Wokan Is., 9 April 1873; collected by 0. Beccari (field number 157); from G. Doria;

SYNTYPE; MZUT Av9963 - mounted skin; adult female; Indonesia, Aru Is., Giabu-Lengan, 12 May 1873; collected by O. Beccari (field number 310); from G. Doria.

Notes: these syntypes are cited by Salvadori (1915: 30), but they are not mentioned in the index of the same work.

\section{CRACIDAE}

Penelope aequatorialis Salvadori \& Festa, 1900

Salvadori T., Festa E., 1900. Boll. Mus. Zool. An. comp. Univ. Torino, No. 368 (vol. XV): 38. Now: Penelope purpurascens aequatorialis Salvadori \& Festa, 1900

SYNTYPE; MZUT Av6479 - skin; adult male; Ecuador, Rio Peripa, November 1897; collected by E. Festa (field number 2501);

SYNTYPE; MZUT Av6480 - skin; adult male; Ecuador, Rio Peripa, November 1897; collected by E. Festa (field number 2625);

SYNTYPE; MZUT Av6481 - skin; adult female; Ecuador, Rio Peripa, November 1897; collected by E. Festa (field number 2500);

SYNTYPE; MZUT Av9351 - skin; adult male; Ecuador, November 1897; collected by E. Festa (field number 2496). 
Notes: the specimen Av9351 is not recorded in old Museum Catalogues and is not cited in Salvadori (1915), but it is quoted as syntype with number A-1040 by Elter (1986: 350).

\section{PHASIANIDAE}

Caccabis callolaema Salvadori \& Festa, 1916

Salvadori T., Festa E., 1916. Boll. Mus. Zool. Anat. Comp. Torino, No. 714 (vol. XXXI): 2.

Now: Alectoris barbara barbata (Reichenow, 1896)

SYNTYPE; MZUT Av13041 - mounted skin; adult male; Lybia, Cyrenaica, Jebel El-Akhdar, no date; donated by E. Festa;

SYNTYPE; MZUT Av13042 - mounted skin; adult female; Lybia, Cyrenaica, Jebel ElAkhdar; no date; donated by E. Festa;

SYNTYPE; MZUT Av7952 - skin; adult female; Lybia, Benghazi; donated by E. Festa.

Perdix rubricollis Cretzschmar, 1829

Cretzschmar P. J., 1829. Atlas zu der Reise im nördlichen Afrika von Eduard Rüppell, Part 14, Pag. 44, Plate 30.

Francolinus leucoscepus G. R. Gray, 1867

Gray G. R., 1867. List of the Specimens of Birds in the Collection of the British Museum, Part V, pag. 48.

Now: Pternistis leucoscepus (G.R. Gray, 1867)

SYNTYPE (possible); MZUT Av2631 - mounted skin; adult; Ethiopia, Abyssinia, no date; collected by Rüppell on an unspecified date and donated in 1839 to the Turin museum.

Notes: according to Salvadori (1915: 10-11) all the specimens sent by Rüppell to the Museum of Turin in 1839 had been collected in Abyssinia in 1832. If this were true, the specimen Av2631 could not be a type. It is possible, however, that the specimen derives from an early trip of Rüppell and that it was to Cretzschmar's disposal in 1829: in this case the specimen would be a syntype of both P. rubricollis Cretzschmar, 1829 and nomen novum Francolinus leucoscepus GR Gray, 1867 (Frank D. Steinheimer, pers. comm. to G. Aimassi, 1 October 2015). The MS. catalogue of the MZUT collection reads "Abissinia / Da M. Rüppell"; "nel 1839" was added subsequently.

Two syntypes are in the Senckenberg Museum, Frankfurt am Main, and two "arguable" syntypes are in the Natural History Museum, Tring (Steinheimer 2005a: 6).

Francolinus castaneicollis Salvadori, 1888

Salvadori T., 1888, Ann. Mus. Civ. Genova, Ser. 2a 6 (XXVI): 542.

Now: Pternistis castaneicollis castaneicollis (Salvadori, 1888)

HOLOTYPE; MZUT Av10690 - mounted skin; immature female; Ethiopia, 'Ciar-Ciar' lake, 22 December 1886; collected by V. Ragazzi and donated by the Società Geografica Italiana. Notes: Salvadori (1888a: 542) erroneously reports 1888 as the date of capture, but in the same work, on p. 525, we read that all the specimens were collected from 12 November 1886 to February 1887.

Francolinus spilogaster Salvadori, 1888

Salvadori T., 1888, Ann. Mus. Civ. Genova, Ser. 2a, 6 (XXVI): 541.

Now: Dendroperdix sephaena spilogaster (Salvadori, 1888)

HOLOTYPE; MZUT Av10689 - mounted skin; adult male; Ethiopia, Harari, 30 January 1887; collected by V. Ragazzi and donated by the Società Geografica Italiana.

\section{PODICIPEDIDAE}

Podiceps capensis Salvadori 1884

Salvadori T., 1884. Ann. Mus. Civ. Genova, Serie 2a, Vol. 1 (XXI): p. 252-254.

Now: Tachybaptus ruficollis capensis (Salvadori, 1884)

SYNTYPE; MZUT Av4185 - dismounted skin; unsexed adult; no locality, no date; donated by Ricord in 1842; 
SYNTYPE; MZUT Av10423 - dismounted skin; adult female; Ethiopia, “Galla, Rugghiè”, 26 March 1879; collected by 0. Antinori (field number 816);

SYNTYPE; MZUT Av10424 - dismounted skin; adult male; Ethiopia, Shewa, "Cialalakà" Lake (Cheleleka or Dabashi Lake), 6 April 1879; collected by 0. Antinori (field number 826);

SYNTYPE; MZUT Av10460 - dismounted skin; adult male; Ethiopia, “Galla, Rugghiè”, 26 March 1879; collected by 0. Antinori (field number 815).

Notes: Salvadori (1884: 252) was aware that Lichtenstein and Bonaparte had used this name for many years (Podiceps capensis in Lichtenstein, 1854: 104; Tachybaptus capensis Bp. in Bonaparte, 1856: 775), but also that they had never described it. However, a few years after having officially introduced this taxon, Salvadori (1888: 326) indicated it as "Tachybaptes (sic) capensis (Licht.)"; moreover, he did not list the types housed in the Collection of the Turin Museum (Salvadori 1915). Elter (1986: 376-377) correctly ascribed capensis to Salvadori, but did not report the specimens of Turin as types.

Specimens MZUT Av10423, Av10424, Av10460 were all collected by 0. Antinori and are easily recognizable within those mentioned in Salvadori (1884). Av4185 is quoted at the end of the description: neither locality nor collecting date are known, but the specimen was donated to the Museum of Turin by Mr. Ricord in 1842. An old label reads: "Podiceps minor, Lath. In ab. estivo / Ricord". We cannot therefore confirm that the specimen obtained from Ricord was already named P. minor capensis, as claimed by Salvadori (1884: 254).

Arbocco et al. (1986: 15) mention a syntype in the Genoa Museum.

Podiceps affinis Salvadori, 1866

Salvadori T., 1866. Atti Soc. it. Sc. Nat, VIII [1865], fasc. 4: 384-387.

Now: Podiceps grisegena holbollii Reinhardt, 1854

HOLOTYPE; MZUT Av3117 - immature; dismounted skin; N America, no date; purchased from Heinrich Vogt in 1833.

Notes: the reference paper of Salvadori is included in the "fascicolo" 4, issued in February 1866 (Galbreath et Aimassi 2020: 147).

Podiceps infuscatus Salvadori, 1884

Salvadori T., 1884, Ann. Mus. Civ. Genova, Serie 2a 1 (XXI): 251-252.

Now: Podiceps cristatus infuscatus Salvadori, 1884

SYNTYPE; MZUT Av10421 - dismounted skin; male; Ethiopia, Shewa, 'Kilohè Lake', May 1881; collected by O. Antinori;

SYNTYPE; MZUT Av10422 - dismounted skin; female; Ethiopia, Shewa, 'Kilohè Lake', May 1881; collected by O. Antinori.

\section{COLUMBIDAE}

Streptopelia barbarù Antinori, 1864

Antinori 0., 1864 - Catalogo descrittivo di una collezione di uccelli fatta da Orazio Antinori [...]: 89-90.

Now: Streptopelia vinacea (J. F. Gmelin, 1789)

HOLOTYPE; MZUT Av5528 - mounted skin; male; Ethiopia, Nguri, April 1861; collected by 0 . Antinori.

Macropygia modiglianii Salvadori, 1887

Salvadori T., 1887. Ann. Mus. Civ. Genova, XXIV: 559-561.

Now: Macropygia emiliana modiglianii Salvadori, 1887

SYNTYPE; MZUT Av10599 - dismounted skin; female; Indonesia, Nias Is., 27 July 1886; collected by E. Modigliani.

Macropygia cinnamomea Salvadori, 1892

Salvadori T., 1892. Ann. Mus. Civ. Genova, Serie 2a 12 (XXXII): 140-141. 
Now: Macropygia emiliana cinnamomea Salvadori, 1892

SYNTYPE; MZUT Av11402 - dismounted skin; female; Indonesia, Enggano Is., Bua-bua, 30 May 1891; collected by E. Modigliani.

Reinwardtoenas reinwardti griseotincta E. Hartert, 1896

Hartert E. in Rothschild W. \& Hartert E., 1896. Novitates Zoologicae, 3: 18-19.

Now: Reinwardtoena reinwardti griseotincta E. Hartert, 1896

PARALECTOTYPE; MZUT Av9222 - mounted skin; male; Indonesia, New Guinea, Andai, October 1872; collected by L.M. D’Albertis; from Genoa Museum.

Notes: according to the ICZN (1999, Art. 72.4.1), the bibliographic refence by Hartert (1896: 19) to the Papuan specimens discussed by Salvadori in his Ornitologia della Papuasia e delle Molucche, vol. III, p. 128, allows to include specimen MZUT Av9222 (quoted under letter "a", p. 126) in the type series. A similar argument was proposed by Arbocco et al. (1986: 19) for the 10 syntypes of the Genoa Museum. However, if we consider the indication of the "type" by Hartert (1928: 8) as a lectotype designation, the Turin and Genoa specimens should be considered paralectotypes.

Columba tucumana Salvadori, 1895

Salvadori T., 1895. Boll. Mus. Zool. An. comp. Univ. Torino, No. 208 (vol. X): 22-23.

Now: Patagioenas fasciata albilinea (Bonaparte, 1854)

HOLOTYPE; MZUT Av11353 - mounted skin; immature male; Argentina, Tucuman, San Pablo, December 1893; collected by A. Borelli.

Notes: Peters (1937: 68, footnote 1) states: "As near as can be determined without actually examining the type, tucumana is based on an aberrant specimen of albilinea. A single specimen of albilinea in the Museum of Comparative Zoölogy from Tucumàn is very close to the typical subspecies, but slightly larger."

Homoptila decipiens Salvadori, 1871

Salvadori T., 1871. Atti R. Acc. Sc. Torino, vol. 6: 131-132.

Now: Leptotila verreauxi decipiens (Salvadori, 1871)

SYNTYPE; MZUT Av5776 - dismounted skin; male; Brazil, no date; donated by "sig. Comm. Cerruti", Italian consul in Bahia, Brazil, in 1863.

Leptoptila callauchen Salvadori, 1897

Salvadori T., 1897. Boll. Mus. Zool. An. comp. Univ. Torino, No. 292 (vol. XII): 33.

Now: Leptotila verreauxi chalcauchenia Sclater \& Salvin, 1870

SYNTYPE; MZUT Av6416 - skin; male; Argentina, Jujuy, San Lorenzo, May 1896; collected by A. Borelli;

SYNTYPE; MZUT Av6417 - skin; male; Argentina, Jujuy, San Lorenzo, May 1896; collected by A. Borelli.

Leptoptila chlorauchenia Giglioli \& Salvadori 1870

Giglioli E. H., Salvadori T., 1870. Ibis, n.s., VI: 186-187.

Now: Leptotila verreauxi chalcauchenia Sclater \& Salvin, 1870

HOLOTYPE; MZUT Av6105 - dismounted skin; Uruguay, Montevideo, 24 January 1866; collected by E.H. Giglioli.

Notes: the description of L. chlorauchenia was presented at the Academy of Sciences of Turin in the session of January 2, 1870, but the publication of the related Atti (Giglioli et Salvadori 1870a) could follow that on Ibis (Giglioli et Salvadori 1870b), dated with certainty in April 1870 .

According to Richmond (in Peters 1923: 200), the description of L. chlorauchenia Giglioli \& Salvadori in the Atti della Reale Accademia delle Scienze of Turin was mentioned for the first time in the Journal für Ornithologie of July 1870. This quotation makes it possible to establish that the work was published before that date but does not allow the claim that it was published after the month of March nor, as Peters (1937: 124) maintains, "after April". Therefore, noth- 
ing can be inferred about the possible priority of L. chalcauchenia Sclater \& Salvin, published in P.Z.S. in April (Duncan 1937: 72).

Wetmore (1926: 176-177) and Hellmayr et Conover (1942: 583) give priority to L. chlorauchenia Giglioli \& Salvadori. Peters (1923: 200; 1937: 124) gives priority to L. chalcauchenia Sclater \& Salvin. Dickinson et Remsen (2013: 61) adopt Peters' choice, however they admit that the situation is not clarified.

Leptoptila saturata Salvadori, 1897

Salvadori T., 1897. Boll. Mus. Zool. An. comp. Univ. Torino, No. 292 (vol. XII): 33-34.

Now: Leptotila megalura Sclater \& Salvin, 1879

SYNTYPE; MZUT Av6405 - skin; male; Argentina, Jujuy, San Lorenzo, June 1896; collected by A. Borelli (field number 455);

SYNTYPE; MZUT Av6406 - skin; male; Argentina, Jujuy, San Lorenzo, May 1896; collected by A. Borelli (field number 456);

SYNTYPE; MZUT Av6407 - skin; immature male; Argentina, Salta, Cara-huassi, 1896; collected by A. Borelli (field number 679).

Notes: the three specimens correspond to those quoted by Salvadori (1897a: 33-34), however Salvadori (1915: 32) avoids mentioning specimen Av6404 among the types and specimen Av6407 is not cited as a type by Elter (1986: 271). "S populations sometimes awarded separate race, saturata" (del Hoyo et Collar 2014: 172).

Carpophaga oenothorax Salvadori, 1892

Salvadori T., 1892. Ann. Mus. Civ. Genova, Serie 2a, Vol. 12 (XXXII): 139-140.

Now: Ducula aenea oenothorax (Salvadori, 1892)

SYNTYPE; MZUT Av11389 - dismounted skin; male; Indonesia, Enggano Is., "Kifa-juc", 7 May 1891; collected by E. Modigliani.;

SYNTYPE; MZUT Av11390 - dismounted skin; Indonesia, Enggano Is., "Kifa-juc", 7 May 1891; collected by E. Modigliani.

Gymnophaps albertisii Salvadori, 1874

Salvadori T., 1874. Ann. Mus. Civ. Genova, VI: 86-87.

Now: Gymnophaps albertisii albertisii Salvadori, 1874

SYNTYPE; MZUT Av9221 - dismounted skin; M; Indonesia, Irian Jaya Barat, Andai, August 1872; collected by L.M. D’Albertis; from Genoa Museum.

Notes: Salvadori (1915: 29) considers specimen Av9221 just a "topotype".

\section{CAPRIMULGIDAE}

Stenopsis macrorrhyncha Salvadori, 1868

Salvadori T., 1869. Atti Soc. it. Sc. Nat., XI [1868], fasc. 3: 447-448.

Now: Systellura longirostris decussata (von Tschudi, 1844)

SYNTYPE; MZUT Av4638 - dismounted skin; adult male; "S America"; obtained from "Colombi" (from Brest, France); the date February 1845 most likely refers to the purchase of the specimen;

SYNTYPE; MZUT Av4639 - skin; immature male?; S America, February 1845; obtained from "Colombi" (from Brest, France); the date February 1845 most likely refers to the purchase of the specimen.

Notes: see the notes below, under Scotornis nigricans.

Caprimulgus fraenatus Salvadori, 1884

Salvadori T., 1884, Ann. Mus. Civ. Genova, Serie 2a, Vol. 1 (XXI): 118-119.

Now: Caprimulgus fraenatus Salvadori, 1884

HOLOTYPE; MZUT Av2697 - skin; male; Ethiopia, Shewa, Daimbi, February 1882; collected by 0 . Antinori.

Notes: Salvadori (1915: 32) and Elter (1986: 115) also mention specimen Av2701 as a type, but Salvadori (1884: 119) has explicitly described a single bird. 
Scotornis nigricans Salvadori, 1868

Salvadori T., 1869. Atti Soc. it. Sc. Nat., XI [1868], fasc. 3: 449-450.

Now: Caprimulgus climacurus nigricans (Salvadori, 1868)

HOLOTYPE; MZUT Av5618 - dismounted skin; adult male; Sudan, White Nile, no date; collected by A. Brun-Rollet.

Notes: this species, together with Stenopsis macrorrhyncha, was presented on 16 September 1868 at the "Terza riunione straordinaria della Società Italiana di Scienze Naturali, tenuta in Vicenza", but the proceedings of the meeting were published in the "fascicolo" No. 3 of the Atti della Società Italiana di Scienze Naturali, Vol. XI, issued on February 1869 (Salvadori 1869a; Galbreath et Aimassi 2020: 147). However, the finding of a separately printed paper dated 1868 (R. Galbreath, pers. comm.) can confirm the date adopted so far.

\section{APODIDAE}

Cypselus myoptilus Salvadori, 1888

Salvadori T., 1888. Ann. Mus. Civ. Genova, XXVI: 228-29.

Now: Schoutedenapus myoptilus myoptilus (Salvadori, 1888)

HOLOTYPE; MZUT Av2731 - skin; female; Ethiopia, Let-Marefià, 10 September 1886; collected by V. Ragazzi (field number 853).

Cypselus shelleyi Salvadori, 1888

Salvadori T., 1888. Ann. Mus. Civ. Genova, XXVI: 227-28.

Now: Apus niansae niansae (Reichenow, 1887)

SYNTYPE; MZUT Av2728 - skin; Ethiopia, Shewa, Daimbi, 16 June 1885; collected by V. Ragazzi (field number 233).

Notes: Salvadori (1915: 34) also reports a second syntype (Ragazzi collection, field number 232), which is no longer present in the MZUT collection.

\section{TROCHILIDAE}

Mellisuga salvadorii Benvenuti, 1863

Benvenuti E., 1863. Descrizione di quattro nuove specie della famiglia dei Trochilidi, provenienti dalla Nuova Granata e di una nuova specie di Sylvia del Brasile. Firenze, Stamperia Reale, p. 13.

Now: Aglaiocercus kingii kingii (Lesson, 1832)

HOLOTYPE; MZUT Av6213 - mounted skin; female; Republic of Colombia, "Nuova Granata" / "Bogotà"; purchased in 1866 from the heirs of Enrico Benvenuti.

Notes: some authors considered that the original description of this species was to be referred to a later work (Benvenuti 1866). Salvadori (1918: 28-29) clarified the misunderstanding and proposed the synonymy with "Cyanolesbia forficata ${ }_{+}$.

Mellisuga ridolfii Benvenuti, 1863

Benvenuti E., 1863. Descrizione di quattro nuove specie della famiglia dei Trochilidi, provenienti dalla Nuova Granata e di una nuova specie di Sylvia del Brasile. Firenze, Stamperia Reale, p. 15.

Now: Eriocnemis vestita vestita (Lesson, 1839)

HOLOTYPE; MZUT Av6209 - mounted skin; female; Republic of Colombia, "Nuova Granata" / "Bogotà"; purchased in 1866 from the heirs of Enrico Benvenuti.

Notes: some authors considered that the original description of this species was to be referred to a later work (Benvenuti 1866). Salvadori (1918: 28-29) clarified the misunderstanding and proposed the synonymy with "Eriocnemis vestita $\bigcirc$ ".

Mellisuga judith Benvenuti, 1863

Benvenuti E., 1863. Descrizione di quattro nuove specie della famiglia dei Trochilidi, provenienti dalla Nuova Granata e di una nuova specie di Sylvia del Brasile. Firenze, Stamperia Reale, p. 11. 
Now: Boissonneaua flavescens flavescens (Loddiges, 1832)

HOLOTYPE; MZUT Av6219 - skin; Colombia, Republic of Colombia, "Nuova Granata" / "Bogotà"; purchased in 1866 from the heirs of Enrico Benvenuti.

Notes: some authors considered that the original description of this species was to be referred to a later work (Benvenuti 1866). Salvadori (1918: 28-29) clarified the misunderstanding and proposed the synonymy with "Panoplites flavescens".

Polytmus ceciliae Benvenuti, 1863

Benvenuti E., 1863. Descrizione di quattro nuove specie della famiglia dei Trochilidi, provenienti dalla Nuova Granata e di una nuova specie di Sylvia del Brasile. Firenze, Stamperia Reale, p. 9.

Now: Campylopterus falcatus (Swainson, 1821)

HOLOTYPE; MZUT Av6227 - mounted skin; female; Republic of Colombia, "Nuova Granata" / "Bogotà"; purchased in 1866 from the heirs of Enrico Benvenuti.

Notes: some authors considered that the original description of this species was to be referred to a later work (Benvenuti 1866). Salvadori (1918: 28-29) clarified the misunderstanding and proposed the synonymy with "Campylopterus lazulus $ᄋ$ ".

\section{CUCULIDAE}

Polophilus nigricans Salvadori, 1876

Salvadori T., 1876. Ann. Mus. Civ. Genova, IX: 17-18.

Now: Centropus phasianinus nigricans (Salvadori, 1876)

SYNTYPE; MZUT Av10186 - mounted skin; adult female; New Guinea, Naiabui, September 1875; collected by L.M. D’Albertis; from G. Doria.

Notes: in Salvadori (1880b: 33) the collection date is "September 1874" (error).

\section{RALLIDAE}

Hypotaenidia saturata Sclater, 1880

Sclater P. L., 1880. The Ibis, 4th ser., IV: 310.

Rallus torquatus limarius Peters, 1934

Peters J. L., 1934. Check-list of Birds of the World. Vol. II: 166.

Now: Gallirallus torquatus limarius (Peters, 1934)

SYNTYPE (possible); MZUT Av18657 (former 6597) - skin; female; Indonesia, Salawatti, 21 July 1875 ; collected by 0 . Beccari.

Notes: Sclater (1880: 310) attributed to this species the three specimens that Salvadori (1876a: 976) previously identified as H. celebensis?, however he only examined one specimen (almost certainly a male housed in the Museo Civico of Genoa; E. Borgo, pers. comm., Arbocco et al. 1979: 192), which Salvadori had already sent him with the name Hypotaenidia saturata.

Rallus torquatus limarius Peters (1934: 166) is a nomen novum for Hypotaenidia saturata "Salvadori" Sclater 1880 (published 4 August), pre-occupied by Rallus longirostris saturatus Ridgway 1880 (published 6 July).

Gallinula galeata Wied, 1833

Wied M., 1833. Beiträge zur Naturgeschichte von Brasilien. Band IV: 807.

Now: Gallinula galeata galeata (M. H. C. Lichtenstein, 1818)

SYNTYPE; MZUT Av15876 (former 3080) - dismounted skin; adult female; Brazil, no date; collected by M. Wied; donated in 1823.

Notes: in the MS. catalogue we read "Gallinula galeata, Wied. Beitr. Z. Bras. T. 4. p. $807 \mathrm{f}$ Brasile. Ricevuta dal Principe Massimiliano di Neu-Vied (sic) al suo ritorno dal Brasile. In 1823". The specimen also bears a label with the inscription "Gallinula galeata (Licht.) F Brasile (V ${ }^{\circ}$ Pr.pe di Neu-Vied)". 
According to Salvadori (1915: 8) all the specimens donated by Maximilian zu WiedNeuwied to Bonelli in 1823 are to be considered "cotypes" of the species he published many years later. Despite this, Elter (1986: 216) does not report the specimen Av15876 as a type.

The description by Wied (1833: 807) took place ten years after the donation to the Turin Museum, but the specimen Av15876 had already been donated with the name Gallinula galeata. For this reason, it seems correct to consider this specimen as a syntype.

G. galeata Wied is not a valid name because it is a junior synonym of Crex galeata Lichtenstein (1818: 36, quoted in Sherborn 1926b: 2622). When the species galeata Lichtenstein was included in the gen. Gallinula (as in Sclater et Salvin 1868b: 462), Gallinula galeata Wied has also become a junior (secondary) homonym.

There are no type specimens of G. galeata in the American Museum of Natural History, where the zoological collections of Wied are hosted (Allen 1889, Greenway 1973), nor among the most important European collections (van den Hoek Ostende et al. 1997, Schifter et al. 2007, Voisin et Voisin 2015, Warren 1966, S. Frahnert, pers. comm. to G. Aimassi, 9 February 2016), therefore the MZUT specimen Av15876 could be the only syntype still existing.

Porphyriops leucopterus Salvadori, 1866

Salvadori T., 1866. Atti Soc. it. Sc. Nat., vol. VIII [1865], fasc. 4: 382-384.

Now: Gallinula melanops melanops (Vieillot, 1819)

SYNTYPE; MZUT Av3655 - dismounted skin; S America; from "Sig. Ferraris" in 1843;

SYNTYPE; MZUT Av4405 - dismounted skin; adult; Argentina; from "Sig. Ferraris" in 1843.

Notes: the reference paper of Salvadori is included in the "fascicolo" 4, issued in February 1866 (Galbreath et Aimassi 2020: 147). In Salvadori (1866a: 382) the specimen Av3655 is erroneously quoted with the number 3635 . The synonymy was already reported by Sharpe (1894: 182).

\section{MUSOPHAGIDAE}

Corythaix leucotis Rüppell, 1835

Rüppell E., 1835. Neue Wirbelthiere zu der Fauna von Abyssinien gehörig. Vögel, pag. 8-9, taf. 3 .

Now: Tauraco leucotis leucotis (Rüppell, 1835)

PARALECTOTYPE; MZUT Av1755 - mounted skin; Ethiopia, Abyssinia, August 1832; from Rüppell coll. in 1839 (field number 189).

Notes: the specimen Av1755 was collected by Rüppell in Abyssinia in 1832 and was sent to the Turin Museum in 1839; it was referred to as "cotype" by Salvadori (1915: 10-11). The Senckenberg-Museum Frankfurt am Main (SMF) holds the lectotype (Steinheimer 2005a: 25).

\section{OCEANITIDAE}

Fregetta melanoleuca Salvadori, 1908

Salvadori T., 1908. Bull. B.O.C., Vol. 21 (N. CXLII): 78.

Now: Fregetta tropica melanoleuca Salvadori, 1908

HOLOTYPE; MZUT Av3256 - mounted skin; Tristan d'Acunha Island, no date; from Bullock coll. in 1819 .

Notes: in his handwritten catalogue F.A. Bonelli says that he showed this bird to C.L. Bonaparte, who did not notice its peculiarities. Despite having been studied by Bonelli and Bonaparte, this specimen had to wait almost a century before being recognized as belonging to a new species. Sharpe (1908: 80) reports that he has found no evidence of this specimen in the Bullock sales catalogue.

\section{DIOMEDEIDAE}

Thalassogeron desolationis Salvadori, 1911

Salvadori T., 1911. Boll. Mus. Zool. An. comp. Univ. Torino, No. 638 (vol. XXVI): 1-2.

Now: Thalassarche chrysostoma (J. R. Forster, 1785) 
HOLOTYPE; MZUT Av7667 - mounted skin; female; Kerguelen Islands, 11 November 1869; from C. Regnoli coll.

Notes: the MS. catalogue notes: "Diomedea plateni female (teste Rothschild) desolationis, Salvad. Typus [...] Desperation Island at entrance of [Magellan] Strait from Pacific side." On the original label written by C. Regnoli we read "? Diomedea culminata (Gould) $£ 20$ [...] 1869 [...] Collezione Regnoli" and, on the opposite side, "Stretto Magellano Isola [...] ".

\section{PROCELLARIIDAE}

Estrelata defilippiana Giglioli \& Salvadori, 1868

Giglioli E.H., Salvadori T., 1868. Nuove specie di Procellaridi raccolte durante il viaggio fatto intorno al mondo negli anni 1865, 1866, 1867, 1868 dalla pirocorvetta italiana Magenta ("Estratto dagli Atti della Società italiana di scienze naturali, Vol. XI, fasc. III"). Milano, Bernardoni, $9 \mathrm{p}$.

Now: Pterodroma defilippiana (Giglioli \& Salvadori, 1869)

SYNTYPE; MZUT Av18779 (former 6730) - mounted skin; Southern Pacific Ocean, 1867; collected by E.H. Giglioli;

SYNTYPE; MZUT Av18780 (former 6732) - mounted skin; Southern Pacific Ocean, 1867; collected by E.H. Giglioli.

Notes: this new species, together with the following Astrelata arminjoniana, Estrelata trinitatis, Astrelata magentae and Puffinus elegans, was presented on 16 September 1868 at the "Terza riunione straordinaria della Società Italiana di Scienze Naturali, tenuta in Vicenza". The proceedings of the meeting were published in the "fascicolo" No. 3 of the Atti della Società Italiana di Scienze Naturali, Vol. XI, issued on February 1869 (Giglioli et Salvadori 1869a; Galbreath et Aimassi 2020: 147), about a month after the publication of the English translation on The Ibis (Giglioli et Salvadori 1869b), which has so far been considered as the reference paper.

However, a separately printed paper dated 1868 has recently been identified from scans of copies held by the library of the Royal Ontario Museum (R. Galbreath, pers. comm.). For this reason, the description paper and the dates of the species have been revised.

Estrelata arminjoniana Giglioli \& Salvadori, 1868

Giglioli E.H., Salvadori T., 1868. Nuove specie di Procellaridi raccolte durante il viaggio fatto intorno al mondo negli anni 1865, 1866, 1867, 1868 dalla pirocorvetta italiana Magenta ("Estratto dagli Atti della Società italiana di scienze naturali, Vol. XI, fasc. III"). Milano, Bernardoni, $9 \mathrm{p}$.

Now: Pterodroma arminjoniana arminjoniana (Giglioli \& Salvadori, 1869)

SYNTYPE; MZUT Av6687 - mounted skin; Brazil, Is. of Trindade (20³1’S, 29¹9’W), 23 January 1868; collected by E.H. Giglioli;

SYNTYPE; MZUT Av6688 - mounted skin; Brazil, Is. of Trindade (20³1’S, 29¹9’W), 23 January 1868; collected by E.H. Giglioli.

Notes: for the dating of the species, see the notes above, under Astrelata defilippiana.

Astrelata trinitatis Giglioli \& Salvadori, 1868

Giglioli E.H., Salvadori T., 1868. Nuove specie di Procellaridi raccolte durante il viaggio fatto intorno al mondo negli anni 1865, 1866, 1867, 1868 dalla pirocorvetta italiana Magenta ("Estratto dagli Atti della Società italiana di scienze naturali, Vol. XI, fasc. III"). Milano, Bernardoni, $9 \mathrm{p}$.

Now: Pterodroma arminjoniana arminjoniana (Giglioli \& Salvadori, 1869)

SYNTYPE; MZUT Av6685 - mounted skin, dark morph; Brazil, Is. of Trindade (20³1'S, $29^{\circ} 19^{\prime}$ W), 23 January 1868; collected by E.H. Giglioli;

SYNTYPE; MZUT Av6686 - mounted skin, dark morph; Brazil, Is. of Trindade (20³1'S, $29^{\circ} 19^{\prime}$ W), 23 January 1868; collected by E.H. Giglioli. 
Notes: "only two skins prepared" (Giglioli et Salvadori 1869b: 62; Giglioli, 1870: 40). Astrelata trinitatis corresponds to the dark phase of Pterodroma arminjoniana (cf. Hellmayr et Conover 1948: 81).

For the dating of the species, see the notes above, under Estrelata defilippiana.

OEstrelata externa Salvin, 1875

Salvin 0., 1875, Ibis, third series, Vol. V: 373.

Now: Pterodroma externa (Salvin, 1875)

SYNTYPE (PARALECTOTYPE); MZUT Av8752 - mounted skin; Mas-afuera, no date; from O. Salvin in 1876, ex Leybold coll.

Notes: on the MS. Catalogue we read "Extrelata externa, Salvin (Typus!) Masafuera (Leybold); dal Sig. Salvin in cambio di un Extr. defilippiana". An old label, most likely by Salvin himself, indicates that the specimen is a type and qualifies it as "young".

Salvin (1875: 376) described "one adult and two young". In the Catalogue of the Birds in the British Museum Salvin (1896: 411) quotes two "types of the species", an adult and a "pull. sk." The adult was later indicated by Warren (1966: 94) as holotype but this indication should coincide with a designation of lectotype.

The specimen Av8752 is reported as type by Elter (1986: 61) but, inexplicably, it is not listed in Salvadori (1915).

Estrelata magentae Giglioli \& Salvadori, 1868

Giglioli E.H., Salvadori T., 1868. Nuove specie di Procellaridi raccolte durante il viaggio fatto intorno al mondo negli anni 1865, 1866, 1867, 1868 dalla pirocorvetta italiana Magenta ("Estratto dagli Atti della Società italiana di scienze naturali, Vol. XI, fasc. III"). Milano, Bernardoni, 9 p.

Now: Pterodroma magentae (Giglioli \& Salvadori, 1869)

HOLOTYPE; MZUT Av6689 - mounted skin; South Pacific Ocean, 39³8' S, $125^{\circ} 58^{\prime}$ W, 22 July 1867 ; collected by E.H. Giglioli.

Notes: P. magentae has remained unobserved for a century after its description (see Crockett 1994 and Imber et al. 1998); now held as Critically Endangered by IUCN (del Hoyo et Collar 2014, Ghiraldi et Aimassi 2019: 35-36).

For the dating of the species, see the notes above, under Estrelata defilippiana.

Puffinus elegans Giglioli \& Salvadori, 1868

Giglioli E.H., Salvadori T., 1868. Nuove specie di Procellaridi raccolte durante il viaggio fatto intorno al mondo negli anni 1865, 1866, 1867, 1868 dalla pirocorvetta italiana Magenta ("Estratto dagli Atti della Società italiana di scienze naturali, Vol. XI, fasc. III"). Milano, Bernardoni, 9 p.

Now: Puffinus assimilis elegans Giglioli \& Salvadori, 1869

HOLOTYPE; MZUT Av6085 - mounted skin; South Atlantic Ocean, 43 54' S, $9^{\circ} 20^{\prime}$ E, 2 March 1866; collected by E.H. Giglioli \& F. De Filippi.

Notes: for the dating of the species, see the notes above, under Estrelata defilippiana.

Puffinus baroli Bonaparte, 1857

Bonaparte C. L., 1857. Consp. Gen. Av., II, p. 204.

Now: Puffinus lherminieri baroli Bonaparte, 1857

SYNTYPE; MZUT Av3202 - mounted skin; unknown locality and date; from Baillon coll. in 1820.

Notes: the transfer of this specimen from the Baillon collection to the Turin Museum is also mentioned by Gouraud (2015: 149). The specimen "Mus. Taurin. №. 3202" was explicitly mentioned by Bonaparte (1857: 204). Curiously, Bonaparte attributed to Bonelli the description of P. baroli, however Bonelli attributed the name to Temminck (Salvadori 1872: 299300). The specimen was registered in MS. catalog under Procellaria obscura and is pedestal still bears a label "Puffinus obscurus (Gmel.)". 


\section{ARDEIDAE}

Ardea nigripes Temminck, 1840

Temminck C.-J., 1840. Manuel d’Ornithologie, II éd., Pt. 4, p. 376.

Now: Egretta garzetta nigripes (Temminck, 1840)

SYNTYPE; MZUT Av15625 (former 2792) - mounted skin; Indonesia, Java, no date; from Temminck coll. in 1826.

Notes: the specimen was donated by Temminck long before the description of the species, but it already carried a label with the MS. name. Since no type series was indicated by Temminck (1840: 376), the Turin specimen can be considered a syntype.

Five specimens are hosted in the National Museum of Natural History in Leiden and another in the Naturhistorischen Museum in Vienna (van den Hoek Ostende et al. 1997: 1819, Schifter et al. 2007: 47). The specimen Av15625 is quoted as a type by Elter (1986: 82) but not by Salvadori (1915).

\section{BURHINIDAE}

Oedicnemus indicus Salvadori, 1866

Salvadori T., 1866. Atti Soc. it. Sc. Nat, vol. VIII, fasc. 4: 380-381.

Now: Burhinus oedicnemus indicus (Salvadori, 1865)

SYNTYPE; MZUT Av4086 - dismounted skin; India, "Himalaja" = Uttar Pradesh, Mussoorie, ante 13 February 1841; from Solaroli coll. in 1841;

SYNTYPE; MZUT Av4087 - dismounted skin; India, "Himalaja" = Uttar Pradesh, Mussoorie, ante 13 February 1841; from Solaroli coll. in 1841.

Notes: for further details on locality and capture date see Passerin d'Entrèves et al. (1995: 128-129).

Dickinson et Remsen (2013: 298), according to Peters (1934: 295), indicate 1865 as the date of description, however the original wrapper of the issue n. 4 (pp. 253-508) of vol. VIII of the Atti della Società Italiana di Scienze Naturali is dated February 1866 (Galbreath et Aimassi 2020: 147).

Oedicnemus inornatus Salvadori, 1866

Salvadori T., 1866. Atti Soc. it. Sc. Nat, vol. VIII, fasc. 4: 381-382.

Now: Burhinus senegalensis (Swainson, 1837)

SYNTYPE; MZUT Av1027 - dismounted skin; Sudan, White Nile; collected by A. BrunRollet on an unspecified date and sent to the Turin Museum in 1855;

SYNTYPE; MZUT Av3583 - dismounted skin; Sudan, White Nile; collected by A. BrunRollet on an unspecified date and sent to the Turin Museum in 1855.

Notes: Peters (1934: 295) accepted the subspecies Burhinus senegalensis inornatus (Salvadori), however del Hoyo et al. (1996: 361), Bahr (2011: 20) and Dickinson et Remsen (2013: 198) treat B. senegalensis as monotypic.

Salvadori (1866: 381) explicitly cited as types the specimens Av1027 and Av3583, which at that time were the only ones present in the Turin Museum. Salvadori himself, however, specified that he also examined a specimen, coming from Abyssinia, in the Jardin des Plantes and six specimens in the laboratories of the Frères Verreaux, also in Paris. In Salvadori $(1915: 15,19)$ and in Elter (1986: 331) only these two types are reported, but in the MS. catalogue of the MZUT collection also the specimen No. 7557 (from "Nubia?", no date, ex Verreaux in 1870) is referred to as a type. MZUT Av7557 could be one of the birds that Salvadori (1866: 381) had already examined in Paris, in the storehouse of the Verreaux brothers, before describing the species and therefore could be a syntype. However, there is no evidence to support this hypothesis.

The description date indicated by Peters (1934: 295) is 1865, however Salvadori's reference paper was published in February 1866 (Galbreath et Aimassi 2020: 147). 


\section{CHARADRIIDAE}

Egialites dealbatus Swinhoe, 1870

Swinhoe R., 1870. Proceedings Zoological Society, 138-140.

Now: Charadrius alexandrinus dealbatus (Swinhoe, 1870)

PARALECTOTYPE; MZUT Av11788 (former 6831) - skin; adult male; China, Amoy, 1861; from Giglioli coll., ex R. Swinhoe;

PARALECTOTYPE; MZUT Av18866 (former 6832) - skin; winter plumage; Taiwan, Takao Hill, November 1861; from Giglioli coll., ex R. Swinhoe.

Notes: the lectotype was recently established by Kennerley et al. (2008: 66), who listed a long series of paralectotypes, hosted in several museums. The MRSN of Turin is not mentioned, certainly because neither Salvadori (1915) nor Elter (1986: 59) indicated the MZUT specimens as types. Both Av11788 and Av18866 were known to Swinhoe when he described the new species Agialites dealbatus and Av11788 was collected in the same locality and in the same year as the lectotype.

"The taxa dealbatus and seebohmi are quite different from the northern subspecies; both together or each of them separately may deserve species status, already" (Bahr 2011: 37).

A further paralectotype, whose capture data coincide with those of the birds of the MZUT collection, is hosted at the Genoa Museum (Arbocco et al. 1986: 16).

Lobivanellus melanocephalus Rüppell, 1845

Rüppell E., 1845. Systematische Uebersicht [...], p. 115, pl. 44.

Now: Vanellus melanocephalus (Rüppell, 1845)

PARALECTOTYPE; MZUT Av2754 - dismounted skin; Ethyopia, Abyssinia, 1832; from Rüppell coll. in 1839.

Notes: specimen collected by Rüppell in Abyssinia in 1832, sent to the Turin Museum in 1839 and listed as cotype by Salvadori (1915: 10-11). Not cited as type-specimen by Elter (1986: 478 in genus Tylibyx).

Rüppell collected bird specimens in Africa in 1822-1827 and in 1831-1834 (Steinheimer 2005b: 165). "He gave all bird specimens MS. names, so most of the new names were already on the skins when they arrived at Frankfurt. We know from Leiden and London museums (now Tring) that these specimens were forwarded with the MS. names attached. All those new birds from the 1845 publication were already labelled as new latest in the late 1830s. Therefore [the Turin specimen] is a Paralectotype" (F. D. Steinheimer, pers. comm. to G. Aimassi, 1 October 2015).

The lectotype and one additional paralecotype are at the SMF (Steinheimer 2005a: 8).

\section{THINOCORIDAE}

Thinocorus pallidus Salvadori \& Festa, 1910

Salvadori T., Festa E., 1910. Boll. Mus. Zool. An. comp. Univ. Torino, No. 631 (vol. XXV): $1-2$.

Now: Thinocorus rumicivorus cuneicauda (Peale, 1848)

SYNTYPE; MZUT Av7009 - skin; adult male; Ecuador, Puntilla de S. Elena, January 1898; collected by E. Festa (field number 2860);

SYNTYPE; MZUT Av7010 - skin; male; Ecuador, Puntilla de S. Elena, January 1898; collected by E. Festa (field number 2861);

SYNTYPE; MZUT Av7011 - skin; male; Ecuador, Puntilla de S. Elena, January 1898; collected by E. Festa (field number 2857);

SYNTYPE; MZUT Av7012 - skin; Ecuador, Puntilla de S. Elena, January 1898; collected by E. Festa (field number 2827);

SYNTYPE; MZUT Av7013a - skin; female; Ecuador, Puntilla de S. Elena, January 1898; collected by E. Festa (field number 2863). 
SYNTYPE; MZUT Av7013b - skin; male?; Ecuador, Puntilla de S. Elena, January 1898; collected by E. Festa

Notes: according to Hellmayr et Conover (1948: 235), Dickinson et Remsen (2013: 208, footnote 4), del Hoyo et Collar (2014: 432), T. cuneicauda includes T. pallidus. However, Peters (1934: 307-308) and Bahr (2011: 81) treat separately T. rumicivorus pallidus and T. r. cuneicauda. Specimen MZUT Av7013b is not mentioned in the MS. catalogue; Elter (1986: 460) quotes only 5 catalogue numbers but, correctly, reports the presence of 6 syntypes.

\section{JACANIDAE}

Hydralector novae hollandiae Salvadori, 1882

Salvadori T., 1882. Ornitologia della Papuasia e delle Molucche. Parte III: 309.

Now: Irediparra gallinacea novaehollandiae (Salvadori, 1882)

SYNTYPE; MZUT Av18773 (former 6724) - skin; Australia, no date; collected by L.M. D’Albertis.

Notes: specimen not mentioned in Salvadori (1915) and not considered as a type by Elter (1986: 248). Salvadori (1882a: 309) explicitly states that the new name Hydralector novae hollandiae refers to the two specimens collected by D'Albertis in Australia. The other syntype is housed in the Museum of Genoa, with the catalogue number C.E. 8815 (Arbocco et al. 1979: 192).

\section{SCOLOPACIDAE}

Tringa platyrincha Temminck, 1815

Temminck C.-J., 1815. Manuel d'Ornithologie: 398.

Now: Calidris falcinellus falcinellus (Pontoppidan, 1763)

SYNTYPE (possible); MZUT Av2924 - dismounted skin; Europe, no date; from Temminck (Leiden Museum), in 1826.

Scolopax stenura Bonaparte, 1831

Bonaparte C. L., 1831. Annali di Storia naturale, T. 4, Fasc. XII: 335-336.

Now: Gallinago stenura (Bonaparte, 1831)

SYNTYPE; MZUT Av3021 - dismounted skin; Indonesia, Java, no date; collected by Kuhl \& Hasselt in 1820-21; from Temminck in 1826.

Notes: the oldest MS. catalogue of the Turin Museum, compiled by F.A. Bonelli, reads "N. 3532 Scolopax stenoptera Kuhl". Bonaparte (1831: 335) cited the species Scolopax stenura Kuhl and mentioned an "in litt." communication by Temminck, suggesting the same name. It is almost certain that both Kuhl and Temminck were talking about the specimens housed in Leiden, which initially included the Turin specimen. The specimen Av3021 has already been treated as a syntype by Salvadori (1915: 7) and Elter (1986: 424).

Currently, in the National Museum of Natural History of Leiden there are 6 syntypes (van den Hoek Ostende et al. 1997: 74), some of which have the same origin as the Turin specimen.

\section{ALCIDAE}

Uria craveri Salvadori, 1866

Salvadori T., 1866. Atti Soc. It. Sc. Nat., vol. VIII [1865], fasc. 4: 387-389.

Now: Synthliboramphus craveri (Salvadori, 1865)

LECTOTYPE; MZUT Av5258 - mounted skin; Mexico, Baja California, Natividad Is., 16 June 1857; collected by F. Craveri.

Notes: according to Cooke (1916) Isla Raza is the real type locality of S. craveri, however Violani et Boano (1990), after having examined the documents of F. Craveri (Brizio 1990), 
believe that the locality indicated by Salvadori is correct. More recently, Bowen (2013) has concluded that it is not possible to determine this locality exactly.

When Salvadori (1866: 388) described the new species U. craveri, he declared to have seen both the specimen of the Museum of Turin and other similar specimens, which certainly are those hosted at the "F. Craveri" Museum of Bra and referred to as paratypes by Violani et Boano (1990). As stated by Aimassi et Ghiraldi (2017: 68) the specimen MZUT Av5258 is not the holotype but the lectotype of $U$. craveri, since only in a later work Salvadori (1915: 14) has indicated it as the "type" of the species.

Dickinson et Remsen (2013: 222), according to Peters (1934: 356), indicate 1865 as the date of description, however the date should be corrected to 1866, because the issue $n$. 4, vol. VIII, of the Atti della Società Italiana di Scienze Naturali is dated February 1866 (Galbreath et Aimassi 2020: 147).

\section{LARIDAE}

Sterna leucoptera Temminck, 1815

Temminck C.-J., 1815. Manuel d'Ornithologie: 483-484.

Now: Chlidonias leucopterus (Temminck, 1815)

SYNTYPE (possible); MZUT Av3166 - mounted skin; adult; Switzerland, Geneva Lake, no date; from Temminck coll. in 1818.

Notes: neither Salvadori (1915) nor Elter (1986: 249), who erroneously identifies it as Hydrochelidon nigra, considered this specimen as syntype.

Strictly speaking, it is not possible to prove that the specimen was captured before 1815 , when the species was established, but it is noteworthy that also the two syntypes still present in the National Museum of Natural History in Leiden are not provided with capture date and that they share the same collecting locality (van den Hoek Ostende et al. 1997: 79).

Sterna poliocerca Gould, 1837

Gould J., 1837. Synopsis of the Birds of Australia, Part II, Plate 37.

Now: Thalasseus bergii cristatus (Stephens, 1826)

SYNTYPE (possible); MZUT Av3608 - mounted skin; adult winter; Australia, Tasmania, no date; from J. Gould ex King's College coll., in 1842.

Notes: the specimen is treated as a type by Salvadori (1915: 13) and by Elter (1986: 440). Stone et Mathews (1913: 138) report a "Type in Collection King's College, since lost". Warren (1966: 232) reports a syntype at the British Museum.

\section{ACCIPITRIDAE}

Gyps affricanus Salvadori, 1865

Salvadori T., 1865. Gazzetta Ufficiale del Regno d'Italia, n. 126.

Now: Gyps africanus Salvadori, 1865

SYNTYPE; MZUT Av5491 - mounted skin; adult male; Sudan, Antub (Sennar), September 1859; collected by O. Antinori;

SYNTYPE; MZUT Av5492 - mounted skin; immature; Sudan, Antub (Sennar), September 1859; collected by 0 . Antinori.

Notes: the description of Gyps affricanus (sic) was presented at the Academy of Sciences of Turin in the session of 7 May 1865 and was published for the first time in the Gazzetta Ufficiale del Regno d'Italia ${ }^{1}$ on May 27th. The paper was reprinted twice: in June 1865 in the Rivista delle Alpi, degli Appennini e Vulcani (Salvadori 1865b: 167 - 173) and, four years later, in a volume of the Royal Academy of Sciences of Turin (Salvadori 1869a). Stresemann et Amadon (in Mayr et Cottrell 1979: 305) indicate 1865 as a year of descrip-

1 It was the periodic publication edited by the Kingdom of Italy, which contained legal texts (such as laws and decrees) and other official legal information. 
tion, but erroneously refer to the 1869 volume. The same error was already in Pierantoni (1925: 9).

Gyps erlangeri Salvadori, 1908

Salvadori T., 1908. Boll. Mus. Zool. An. comp. Univ. Torino, No. 576 (vol. XXIII): 1-6.

Now: Gyps rueppelli erlangeri Salvadori, 1908

SYNTYPE; MZUT Av10527 - mounted skin; immature female; Ethiopia, Shewa, LetMarefià, 4 February 1879; collected by 0 . Antinori (field number 792);

SYNTYPE; MZUT Av10678 - mounted skin; adult male; Ethiopia, Shewa, Let-Marefià, 23

November 1884; collected by V. Ragazzi (field number 6);

SYNTYPE; MZUT Av10679 - mounted skin; adult male; Ethiopia, Shewa, Let-Marefià, 17 June 1886; collected by V. Ragazzi (field number 662);

SYNTYPE (possible); MZUT Av283 - skin; adult; Eritrea, Saganeiti, 1899; from R. Gentile coll.;

SYNTYPE (possible); MZUT Av11622 - mounted skin; immature; Eritrea, Saganeiti, 50 km SE di Asmara, 1899; from R. Gentile coll.

Notes: Salvadori (1915: 41) reports 4 "types" of Gyps erlangeri, but indicates three catalogue numbers incorrectly. We believe that the numbers indicated by Salvadori must be corrected as follows: $10628=10678 ; 10629=10679 ; 10622=11622$.

The specimen Av10527 corresponds to the specimen "b" cited by Salvadori (1884: 34) as Gyps fulvus? The specimens Av10678 and Av10679 correspond to the specimens "a" and "b" cited by Salvadori (1888b: 191) as G. rueppelli. These specimens, misidentified in previous works, are part of the type series of $G$. erlangeri because they have been included by Salvadori (1908b: 5) in the new nominal taxon "by bibliographic reference" (ICZN 1999, article 72.4.1).

The specimen Av283 is part of the collection donated by Lieutenant R. Gentile in 1899 (Salvadori 1915: 38) and therefore it was at disposal of Salvadori when he described G. erlangeri; the specimen Av11622, non quoted in the paper of Salvadori has the same capture data as the previous one. Probably both are syntypes.

Elter (1986: 230) only indicates as a type of G. erlangeri the specimen Av10527, the only one certainly mentioned with the right number in Salvadori (1915: 41).

Harpyopsis novae guineae Salvadori, 1875

Salvadori T., 1875. Ann. Mus. Civ. Genova, 7, p. 682-683.

Now: Harpyopsis novaeguineae Salvadori, 1875

PARALECTOTYPE; MZUT Av10117 - mounted skin; adult [male]; Papua New Guinea, 'Baja Hall', 13 April 1875; from G. Doria, ex L. D’Albertis coll.

Notes: the specimen Av10117, captured "not far from the Yule Island", is the second specimen cited by Salvadori (1875a: 683). The first one is housed at the Genoa museum under the number C.E. 24671 (Arbocco et al. 1979: 189). The specimen of Genoa was designated as a lectotype by Salvadori (1880b: 40) (Aimassi et Ghiraldi 2017: 68).

Aquila fasciata Vieillot, 1823

Vieillot L. P., 1823. Encyclopédie Méthodique, II éd., Ornithologie, p. 1192.

Aquila intermedia Boitard, 1824

Boitard M. P., 1824. Histoire naturelle des Oiseaux de proie d'Europe, pp. 24-25, pl. V, fig. 3 Falco bonelli Temminck, 1824

Temminck in Temminck C.-J. \& Laugier de Chartrouse M., 1824. Planches Coloriées, livr. 49, pl. 288

Now: Aquila fasciata fasciata Vieillot, 1822

SYNTYPE; MZUT Av96 - mounted skin; immature female; Italy, Sardinia, 1822; from Cav. Prunner ex A. della Marmora.

Notes: the status of the MZUT Av96 specimen, most likely the only syntype still existing of the three named taxa, has been discussed by Aimassi (2015), who also showed that 
the description date of Aquila fasciata is 1822. Aquila fasciata, Aquila intermedia e Falco bonelli could be treated as objective synonyms because they share at least one specimen (this one) in their type-series. This specimen was depicted by Temminck in the Planches Coloriées (No. 288) and by Biscarra, in the portrait of F. A. Bonelli in 1930, the year of his death.

The specimens Av95, Av97, Av99 and Av100, the last three of which were depicted by Marmora (1834: Pl. I, after page 124), contrary to the opinion of Elter (1986: 205, as Falco bonelli Temminck) are not types.

Urospizias etorques Salvadori, 1876

Salvadori T., 1876. Ann. Mus. Civ. Genova, 7: 901-904.

Now: Accipiter hiogaster leucosomus (Sharpe, 1874)

SYNTYPE; MZUT Av10128 - mounted skin; adult male; New Guinea, Sorong, May 1872; from G. Doria, ex L. D’Albertis coll.;

SYNTYPE; MZUT Av10129 - mounted skin; immature male; New Guinea, Sorong, May 1872; from G. Doria, ex L. D’Albertis coll.;

SYNTYPE; MZUT Av10130 - mounted skin; immature female; New Guinea, Sorong, May 1872; from G. Doria, ex L. D’Albertis coll.

Notes: the date of the original description was corrected according to Poggi (2010:34). Av10128 is the first specimen listed and described by Salvadori (1876a: 901) and corresponds to the specimen "a" cited in Salvadori (1880b: 50); Av10130 is the specimen n. 11 listed by Salvadori (1876a: 903) and corresponds to the specimen "m" in Salvadori (1880b: 52). Arbocco et al. (1979: 188) consider as syntypes 11 specimens housed at the Museo civico di Storia naturale di Genova, however C.E. 21564 and C.E. 21565, cited in Salvadori (1880b), are not part of the 13 specimens of the type series (Salvadori 1876a: 901-903).

\section{Buteo auguralis Salvadori, 1866}

Salvadori T., 1866. Atti Soc. it. Sc. Nat, vol. VIII [1865], fasc. 4: 376-377.

Now: Buteo auguralis Salvadori, 1865

SYNTYPE; MZUT Av4294 - mounted skin; adult; Ethiopia, Abyssinia; from Dr. Bussa in 1842 (but see below);

SYNTYPE; MZUT Av5501 - mounted skin; immature male; Sudan, Kordofan, Gebel Aidun, "Libyan desert", September 1860; collected by O. Antinori (field number 490).

Notes:

Specimen Av4292 - Salvadori (1915: 12) claimed that the specimen had been donated in 1842 by a certain "Dr. Bussa", together with a valuable collection of birds from Abyssinia. We suspect that this collection, together with the one that in the MS. catalogue was attributed to Padre Calvi from Oneglia, actually corresponds to the collection of the missionary and explorer Giuseppe Sapeto of Carcare (1811-1895), who donated it to the Turin Museum, without however receiving any recognition (Sapeto 1857: 272). A drawing of this specimen appearead in Antinori et Salvadori (1874, Plate I).

Specimen Av5501 - According to several indications contained in the "Catalogo descrittivo di una collezione di uccelli d'Affrica" (Antinori 1864), we can deduce that Gebel Aidun is a locality in the "Libyan desert" of Kordofan, which Antinori visited in September, during the third excursion in the territory of Sudan, carried out from 7 August to 13 October 1860. Peters (1931: 230) reports as type-locality of B. auguralis "Abyssinia and Gebel Aidun, Lybian Desert" and Stresemann et Amadon (in Mayr et Cottrell 1979: 375) just quote "Ethiopia". As far as we know, no one has already specified that the type-locality includes the Kordofan region in Sudan.

Dickinson et Remsen (2013: 254), according to Stresemann et Amadon (in Mayr et Cottrell 1979: 375), indicate 1865 as the date of description, however the date should be corrected to 1866, because the issue n. 4, vol. VIII, of the Atti della Società Italiana di Scienze Naturali is dated February 1866 (Galbreath et Aimassi 2020: 147). 


\section{STRIGIDAE}

Pulsatrix fasciativentris Salvadori \& Festa, 1900

Salvadori T., Festa E., 1900. Boll. Mus. Zool. An. comp. Univ. Torino, No. 368 (vol. XV): 32-33.

Now: Pulsatrix melanota melanota (von Tschudi, 1844)

SYNTYPE; MZUT Av389 - dismounted skin; male; Ecuador, Zamora Valley, December 1895; collected by E. Festa;

SYNTYPE; MZUT Av11634 - mounted skin; Ecuador, Zamora Valley, December 1895; collected by E. Festa;

SYNTYPE; MZUT Av11635 - mounted skin; Ecuador, Zamora Valley, December 1895; collected by E. Festa.

Syrnium niasense Salvadori, 1887

Salvadori T., 1887. Ann. Mus. Civ. Genova, XXIV: 526-528.

Now: Strix leptogrammica niasensis (Salvadori, 1887)

SYNTYPE; MZUT Av10597 - mounted skin; adult; Indonesia, Nias Is., 5 May 1886; collected by E. Modigliani.

Notes: in the original description, the collection date is 26 July 1886, while in MS. label is 5 May 1886.

\section{BUCEROTIDAE}

Bycanistes aloysii Salvadori, 1906

Salvadori T., 1906. Boll. Mus. Zool. An. comp. Univ. Torino, No. 542 (vol. XXI): 2.

Now: Bycanistes subcylindricus subquadratus (Cabanis, 1880)

HOLOTYPE; MZUT Av14342 (former 1238) - skin; female; Uganda, Entebbe, August 1906; from Duca degli Abruzzi, ex A. Roccati coll.

\section{PHOENICULIDAE}

Irrisor erythrorhynchos neglectus Neumann, 1905

Neumann 0., 1905. Journ. f. Orn., LIII, Heft I: 194-195.

Now: Phoeniculus somaliensis neglectus Neumann, 1905

HOLOTYPE; MZUT Av2306 - skin; female; Ethiopia, Shewa, Ulà, 29 May 1885; collected by V. Ragazzi;

PARATYPE; MZUT Av2305 - skin; male; Ethiopia, Shewa, Ulà, 6 June 1885; collected by V. Ragazzi;

PARATYPE; MZUT Av2307 - skin; female; Ethiopia, Shewa, Ulà, 29 May 1885; collected by V. Ragazzi;

PARATYPE; MZUT Av15266 (former 2308) - skin; male; Ethiopia, Shewa, Gherba, 26 July 1887; collected by V. Ragazzi;

PARATYPE; MZUT Av15259 (former 2298) - skin; female; Ethiopia, Shewa, Daimbi, 19 November 1879; collected by 0. Antinori;

PARATYPE; MZUT Av2304 - skin; immature male; Ethiopia, Shewa, Daimbi, 19 November 1879; collected by O. Antinori;

PARATYPE; MZUT Av2301 - skin; female; Ethiopia, Shewa, Daimbi, 19 November 1879; collected by 0 . Antinori;

PARATYPE; MZUT Av2299 - skin; Ethiopia, Shewa, Daimbi, 11 November 1879; collected by O. Antinori;

PARATYPE (possible); MZUT Av15263 (former 2303) - skin; immature; Ethiopia, Shewa, Daimbi; 11 November 1879; collected by 0. Antinori;

PARATYPE (possible); MZUT Av2300 - skin; male; Ethiopia, Shewa, Daimbi, 14 April 1879; collected by O. Antinori; 
PARATYPE (possible); MZUT Av2302 - skin; immature; Ethiopia, Shewa, Daimbi, 14 April 1879; collected by 0 . Antinori.

Notes: Neumann (1905) seems to have some doubt in attributing the specimens 867 (Av2302), 868 (Av2300) and 1056 (Av15263) to the new species, nevertheless Salvadori (1915: 32) and Elter (1986: 258) consider them all as "cotypes".

Irrisor cabanisi De Filippi, 1853

De Filippi F., 1853. Rév. et Mag. Zool. pure appl., 2e sér., t. V: 289.

Now: Rhinopomastus minor cabanisi (De Filippi, 1853)

HOLOTYPE; MZUT Av669 - dismounted skin; adult male; Sudan, "Fiume Bianco" [White Nile], from $4^{\circ}$ and $3^{\circ}$ lat. $\mathrm{N}$; collected by A. Brun-Rollet on an unspecified date and sent to the Turin Museum in 1853.

\section{PICIDAE}

Chloronerpes rubripileus Salvadori \& Festa, 1900

Salvadori T., Festa E., 1900. Boll. Mus. Zool. An. comp. Univ. Torino, n. 368 (vol. XV): 14-15. Now: Colaptes rubiginosus rubripileus (Salvadori \& Festa, 1900)

SYNTYPE; MZUT Av728 - skin; male; Ecuador, Rio Peripa, November 1897; collected by E. Festa;

SYNTYPE; MZUT Av729 - skin; female; Ecuador, Vinces, September 1897; collected by E. Festa;

SYNTYPE; MZUT Av730 - skin; female; Ecuador, Vinces, September 1897; collected by E. Festa.

Iyngipicus frater Salvadori \& Giglioli, 1885

Salvadori T., Giglioli E. H., 1885. Atti R. Acc. Sc. Torino, vol. XX: 632-633.

Now: Dendrocopos canicapillus canicapillus (Blyth, 1845)

HOLOTYPE; MZUT Av6177 - mounted skin; adult male; Malaysia? Malay Peninsula, no date; bought in Singapore in 1866, during the Magenta voyage.

Picus (Baeopipo) auratiiventris Salvadori, 1868

Salvadori T., 1868. Atti R. Acc. Sc. Torino, vol. III: 524-525.

Now: Dendrocopos canicapillus aurantiiventris (Salvadori, 1868)

SYNTYPE; MZUT Av6902 - dismounted skin; male; Malaysia, Borneo, Sarawak, 1867; from G. Doria, ex O. Beccari coll.;

SYNTYPE; MZUT Av6903 - dismounted skin; female; Malaysia, Borneo, Sarawak, 1867; from G. Doria, ex O. Beccari coll.

Picus cruentatus Antinori, 1856

Antinori 0., 1856. Naumannia, 6: 411-414.

Now: Dendrocopos syriacus syriacus (Hemprich \& Ehrenberg, 1833)

SYNTYPE; MZUT Av1785; dismounted skin; immature; Syria, 1854; from Antinori collection; Truqui leg.?

Picus khan De Filippi, 1863

De Filippi F., 1863. Arch. Zool. Anat. Fisiol., vol. II, fasc. II: 385.

Now: Dendrocopos syriacus syriacus (Hemprich \& Ehrenberg, 1833)

HOLOTYPE; MZUT Av2163 - dismounted skin; immature; Iran, Teheran, Tedgrish, 1862; collected by F. De Filippi.

\section{RAMPHASTIDAE}

Bucco corvinus Temminck, 1831

Temminck in Temminck C.-J. \& Laugier de Chartrouse M., 1831. Planches Coloriées, livr. 88, pl. 522.

Now: Psilopogon corvinus (Temminck, 1831) 
SYNTYPE; MZUT Av1858 - dismounted skin; adult; Indonesia, Java, no date; from Temminck coll.

SYNTYPE; MZUT Av1859 - dismounted skin; adult; Indonesia, Java, no date; from Temminck coll.

Notes: neither Salvadori (1915) nor Elter (1986: 136, under the name Chotorea corvina), considered these specimens as syntypes. We do not know the date of the transfer of the specimens from the Temmick collection to the Turin Museum, but certainly it precedes the description of the species. Because there is no doubt that the specimens of his own collection were known to Temminck, the two specimens of the MZUT collection have the requisites to be considered syntypes (ICZN 1999, Art. 72.4.1.1). In the Museum of Leiden there is only one syntype, collected by H. Boie (van den Hoek Ostende et al. 1997: 197).

Bucco kotorea Temminck, 1831

Temminck in Temminck C.-J. \& Laugier de Chartrouse M., 1831. Planches Coloriées, livr. 88, text.

Now: Psilopogon javensis (Horsfield, 1821)

SYNTYPE; MZUT Av1860 - dismounted skin; Indonesia, Java, no date; collected by Kuhl \& Hasselt in 1820-21; from Temminck in 1826;

SYNTYPE; MZUT Av1861 - dismounted skin; adult; Indonesia, Java, no date; collected by Kuhl \& Hasselt in 1820-21; from Temminck in 1826.

Notes: in the oldest catalogue of the Turin Museum, in correspondence of N. 3536 (now Av1860), in addition to the MS. data of F. A. Bonelli there is a piece of glued paper, probably the fragment of a letter, which reiterates "Voy. K. et v. H. Bucco Kotorea ${ }^{7}$ ".

Levaillant (1807: 42) in the text of the species "le barbu kotorea male" (Fig. C) transcribed the description communicated to him by Temminck "sans y rien changer, dans la crainte de l'altérer en quoi que ce soit, puisqu'il avoit l'oiseau sous les yeux en le décrivant" (without changing anything, in order not to modify it in any way, because he had the bird under the eyes when he described it). It is therefore evident that Temminck knew the species well before the formal description in the Planches Coloriées (Temminck et Laugier 1831a), even before sending the specimens Av1860 and Av1861 to the Turin Museum (1826). For these reasons the specimens can be considered as syntypes.

Bucco armillaris Temminck, 1821

Temminck in Temminck C.-J. \& Laugier de Chartrouse M., 1821. Planches Coloriées, livr. 15, pl. 89, fig. 1.

Now: Psilopogon armillaris armillaris (Temminck, 1821)

SYNTYPE; MZUT Av1862 - dismounted skin; Indonesia, Java, no date; collected by Kuhl \& Hasselt in 1820-21; from Temminck in 1826.

Notes: a syntype, housed in the museum of Leiden, was collected by Reinwardt (van den Hoek Ostende et al. 1997: 196).

Bucco gularis Temminck, 1821

Temminck in Temminck C.-J. \& Laugier de Chartrouse M., 1821. Planches Coloriées, livr. 15, pl. 89, fig. 2.

Now: Psilopogon australis australis (Horsfield, 1821)

SYNTYPE; MZUT Av14884 (former 1863) - skin; Indonesia, Java, no date; from Temminck in 1822, ex H. Kuhl and J.C. van Hasselt coll.

Notes: two syntypes, housed in the museum of Leiden, were collected by Reinwardt (van den Hoek Ostende et al. 1997: 196);

Capito aequatorialis Salvadori \& Festa, 1900

Salvadori T., Festa E., 1900. Boll. Mus. Zool. An. comp. Univ. Torino, No. 368 (vol. XV): 22. Now: Eubucco bourcierii aequatorialis (Salvadori \& Festa, 1900)

SYNTYPE; MZUT Av704 - skin; adult male; Ecuador, Imbabura, Intag, June 1897; from E. Festa, ex Ollalla coll.; 
SYNTYPE; MZUT Av705 - skin; male; Ecuador, Imbabura, Intag, June 1897; from E. Festa, ex Ollalla coll.

Trachyphonus uropygialis Salvadori, 1894

Salvadori T., 1894. Mem. R. Acc. Sc. Torino, serie 2a, vol. 44: 551.

Now: Trachyphonus darnaudii boehmi Fischer \& Reichenow, 1884

HOLOTYPE; MZUT Av11292 - dismounted skin; adult; Somalia? 1891; collected by E. Ruspoli.

Xylobucco Aloysii Salvadori, 1906

Salvadori T., 1906. Boll. Mus. Zool. An. comp. Univ. Torino, No. 542 (vol. XXI): 2.

Now: Pogoniulus scolopaceus flavisquamatus (J. Verreaux \& E. Verreaux, 1855)

HOLOTYPE; MZUT Av1232 - skin; female; Uganda, Entebbe, 1906; from Duca degli Abruzzi, ex A. Roccati coll.

Lybius undatus salvadorii Neumann, 1903

Neumann O., 1903. Bulletin of the British Ornithologists' Club, XIV: 16.

Now: Lybius undatus salvadorii Neumann, 1903

HOLOTYPE; MZUT Av674 - skin; adult female; Ethiopia, Dinki, 29 October 1885; collected by V. Ragazzi;

PARATYPE; MZUT Av13859 (ex 675); male; Ethiopia, Dinki, 29 October 1885; collected by V. Ragazzi.

Laimodon leucocephalus De Filippi, 1853

De Filippi F., 1853. Rév. et Mag. Zool. pure appl., 2e sér., t. V: 291.

Now: Lybius leucocephalus leucocephalus De Filippi, 1853

HOLOTYPE; MZUT Av3203 - mounted skin; adult; Sudan, "Fiume Bianco" [White Nile], from $4^{\circ}$ and $3^{\circ}$ lat. $\mathrm{N}$; collected by A. Brun-Rollet on an unspecified date and sent to the Turin Museum in 1853.

Pogonias brucii Rüppell, 1837

Rüppell E., 1837. Neue Wirbelthiere zu der Fauna von Abyssinien gehörig. Vögel: pag. 50-51, taf. 20, fig. 1.

Now: Lybius guifsobalito Hermann, 1783

PARALECTOTYPE; MZUT Av1867 - dismounted skin; adult; Ethiopia, Abyssinia, 1832; from Rüppell coll. in 1839.

Notes: for the type status of this specimen, see Steinheimer (2005a: 13).

Pogonias rolleti De Filippi, 1853

De Filippi F., 1853. Rév. et Mag. Zool. pure appl., 2e sér., t. V: 290.

Now: Pogonornis rolleti (De Filippi, 1853)

HOLOTYPE; MZUT Av3207 - dismounted skin; adult male; Sudan, White Nile, from $4^{\circ}$ and $3^{\circ}$ lat. N; collected by A. Brun-Rollet on an unspecified date and sent to the Turin Museum in 1853.

\section{TODIDAE}

Todus hypochondriacus Bryant, 1866

Bryant H., 1866. Proc. Boston Soc. Nat. Hist., X: 250.

Now: Todus mexicanus Lesson, 1838

SYNTYPE; MZUT Av7770 - skin; Puerto Rico, no date; from Smithsonian Inst. in 1871, ex Bryant coll.

Notes: Bryant (1866: 250) claims to have seen "several specimens" but describes in detail the specimen N. 36450. This specimen, quoted by Bangs et Penard (1925: 200) as "Type, formerly in U.S.N.M.", is no longer recorded among the types of the AMNH (Greenway 1978). According to Schifter et al. (2007: 247), all the specimens seen by Bryant are to be regarded as syntypes. 
Bangs et Penard (1925: 197) recall that many birds "described by Dr. Bryant originally went to the U.S.N.M. but subsequently were either donated to or exchanged with home and foreign museums or collectors, and among that number were several of the types, including those of Todus hypochondriachus, [...]".

The specimen Av7770 has not been considered as a type by Salvadori (1915: 27) and by Elter (1986: 466).

\section{ALCEDINIDAE}

Ceyx innominata Salvadori, 1869

Salvadori T., 1869. Atti R. Acc. Sc. Torino, vol. IV: 465-468.

Now: Ceyx erithaca rufidorsa Strickland, 1847

SYNTYPE; MZUT Av15267 (former 2309) - skin; Indonesia, Java; from Prévost coll. in 1827.

Dacelo intermedius Salvadori, 1876

Salvadori T., 1876. Ann. Mus. Civ. Genova, IX: 21-22.

Now: Dacelo leachii intermedia Salvadori, 1876

SYNTYPE; MZUT Av10087 - mounted skin; adult male; New Guinea, Naiabui, September 1875; collected by L.M. D’Albertis; from G. Doria.

\section{FALCONIDAE}

Hypotriorchis ophryophanes Salvadori, 1895

Salvadori T., 1895. Boll. Mus. Zool. An. comp. Univ. Torino, No. 208 (vol. X): 20-21.

Now: Falco rufigularis rufigularis (Daudin, 1800)

SYNTYPE; MZUT Av11358 - mounted skin; adult male; Paraguay, Rio Apa, Colonia Risso, 1893; collected by A. Borelli;

SYNTYPE; MZUT Av11359 - mounted skin; female; Paraguay, Rio Apa, Colonia Risso, 1893; collected by A. Borelli.

Falco frontatus Gould, 1838

Gould J., 1838. Synopsis Birds Australia, Part III, Plate 42.

Now: Falco longipennis longipennis Swainson, 1838

SYNTYPE (possible); MZUT Av3558 - adult; Australia, no date; from J. Gould coll.

\section{PSITTACIDAE}

Pyrrhura borellii Salvadori, 1894

Salvadori T., 1894a. Boll. Mus. Zool. An. comp. Univ. Torino, n. 190 (vol. IX): 3-4.

Now: Pyrrhura frontalis chiripepe (Vieillot, 1818)

HOLOTYPE; MZUT Av11349 - skin; male; Paraguay, Rio Apa, Colonia Risso, 1893; collected by A. Borelli.

Notes: according to Bertoni (1913: 82) the specimens described by Salvadori as P. borellii represent variations of plumage commonly found in Pyrrhura vittata chiripepe.

Pyrrhura hypoxantha Salvadori, 1899

Salvadori T., 1899. Boll. Mus. Zool. An. comp. Univ. Torino, No. 363 (vol. XIV): 1-2.

Now: Pyrrhura molinae hypoxantha Salvadori, 1899

SYNTYPE; MZUT Av11630 - skin; female; Brazil, Mato Grosso do Sul, Urucum, 1899; collected by A. Borelli.

Pyrrhura griseipectus Salvadori, 1900

Salvadori T., 1900. Ibis, 7th ser., VI: 672-673, pl. XIV.

Now: Pyrrhura griseipectus Salvadori, 1900

SYNTYPE; MZUT Av10583 - skin; Brazil? 1888; from M.G. Peracca (from captivity); 
SYNTYPE; MZUT Av10584 - skin; Brazil? 1888; from M.G. Peracca (from captivity).

Notes: the two syntypes of the Turin Museum had been "kept in confinement by Count Peracca" (Salvadori 1900: 673); their locality was unknown, but Salvadori suspected they came from "Guiana".

\section{PSITTACULIDAE}

Nasiterna keiensis Salvadori, 1876

Salvadori T., 1876. Ann. Mus. Civ. Genova, VII [1875]: 984-985.

Now: Micropsitta keiensis keiensis (Salvadori, 1876)

SYNTYPE; MZUT Av9976 - skin; male; Indonesia, Kai Is., 'Kei Ralan', 5 October 1873; collected by 0 . Beccari; from G. Doria.

Platycercus haematogaster Gould, 1838

Gould J., 1838. Proc. Zool. Soc. London, Part V [1837]: 89.

Now: Northiella haematogaster haematogaster (Gould, 1838)

SYNTYPE (possible); MZUT Av3540 - Australia, no date; from J. Gould coll. in 1842.

Notes: as for the other specimens donated by J. Gould in 1842, Av3540 is a possible syntype.

Chalcopsittacus bruijni Salvadori, 1878

Salvadori T., 1878. Atti R. Acc. Sc. Torino, vol. XIII: 310-312.

Now: Chalcopsitta atra insignis Oustalet, 1878

SYNTYPE; MZUT Av9361; 1 ind., Indonesia, Amberpon [Numfor] Is., Geelwink Bay, May 1877; collected by A.A. Bruijn; from E. Turati.

Notes: Salvadori (1878a) does not specify in which collection the two type specimens were housed. However, since a large part of the birds mentioned in the same work was in Turati collection, it is likely that the specimen Av9361 was also located among these specimens.

Elter (1986: 127) considers the specimen n. 9631 as a type, however Salvadori (1915) does not report it as such. The synonymy with Calchopsitta insignis Oustalet is suggested by Salvadori himself (1878a: 312, footnote).

Cyclopsittacus occidentalis Salvadori, 1876

Salvadori T., 1876. Ann. Mus. Civ. Genova, VII [1875]: 910-911.

Now: Psittaculirostris desmarestii occidentalis (Salvadori, 1876)

SYNTYPE; MZUT Av9987 - skin; female; Indonesia, New Guinea, Sorong, June 1872; collected by L.M. D'Albertis; from G. Doria.

Notes: not cited in Salvadori (1915), but listed as type in Salvadori (1880: 153) under "l (218)". Six syntypes are housed in the Genoa Museum (Arbocco et al. 1979: 198).

\section{EURYLAIMIDAE}

Cymborhynchus malaccensis Salvadori, 1874

Salvadori T., 1874. Atti R. Acc. Sc. Torino, 9: 425.

Now: Cymbirhynchus macrorhynchos malaccensis Salvadori, 1874

SYNTYPE; MZUT Av8333 - dismounted skin; adult; Malaysia? Malay Peninsula, no date; collected by E.H. Giglioli \& F. De Filippi during Magenta voyage;

SYNTYPE; MZUT Av8334 - dismounted skin; Malaysia? Malay Peninsula, no date; collected by E.H. Giglioli \& F. De Filippi during Magenta voyage;

SYNTYPE; MZUT Av8336 - dismounted skin; immature; Malaysia? Malay Peninsula, no date; collected by E.H. Giglioli \& F. De Filippi during Magenta voyage.

Notes: specimen MZUT Av8335, a syntype with the same collecting data of those above mentioned, is no longer present in the Turin Museum. 


\section{TITYRIDAE}

Pachyrhamphus xanthogenys Salvadori \& Festa, 1898

Salvadori T., Festa E., 1898. Boll. Mus. Zool. An. comp. Univ. Torino, No. 330 (vol. XIII): 1.

Now: Pachyramphus xanthogenys xanthogenys Salvadori \& Festa, 1898

HOLOTYPE; MZUT Av1352 - skin; adult male; Ecuador, Zamora Valley, December 1895; collected by E. Festa (field number 166).

\section{PIPROMORPHIDAE}

Pseudomyobius annectens Salvadori \& Festa, 1899

Salvadori T., Festa E., 1899. Boll. Mus. Zool. An. comp. Univ. Torino, No. 362 (vol. XIV): 12. Now: Pseudotriccus pelzelni annectens (Salvadori \& Festa, 1899)

HOLOTYPE; MZUT Av1809 - skin; adult; Ecuador, Gualea, July 1897; from E. Festa (field number 987), ex C. Ollalla coll.

Notes: on this species Salvadori based his new Genus Pseudomyobius.

Euscarthmus viridiceps Salvadori, 1897

Salvadori T., 1897. Boll. Mus. Zool. An. comp. Univ. Torino, No. 292 (vol. XII): 12-13.

Now: Poecilotriccus plumbeiceps viridiceps (Salvadori, 1897)

HOLOTYPE; MZUT Av1622 - skin; male; Argentina, Jujuy, S. Lorenzo, May 1896; collected by A. Borelli (field number 419).

\section{TYRANNIDAE}

Serphophaga inornata Salvadori, 1897

Salvadori T., 1897. Boll. Mus. Zool. An. comp. Univ. Torino, No. 292 (vol. XII): 13-14.

Now: Inezia inornata (Salvadori, 1897)

SYNTYPE; MZUT Av1642 - skin; male; Bolivia, Tarija, Villa Ingavi, Rio Pilcomayo, November 1895; collected by A. Borelli (field number 69);

SYNTYPE; MZUT Av1643 - skin; male; Bolivia, Tarija, Villa Ingavi, Rio Pilcomayo, November 1895; collected by A. Borelli (field number 81).

Phyllomyias berlepschi Salvadori, 1897

Salvadori T., 1897. Boll. Mus. Zool. An. comp. Univ. Torino, No. 292 (vol. XII): 13.

Now: Phyllomyias burmeisteri burmeisteri Cabanis \& Heine, 1859

HOLOTYPE; MZUT Av1678 - skin; male; Argentina, Jujuy, San Lorenzo, May 1896; collected by A. Borelli (field number 430).

Notes: Cory et Hellmayr (1927: 480) after examining the type of Phyllomyias berlepschi Salvadori (not Sclater, 1887) in the Turin Museum, established synonymy with "Acrochordopus burmeisteri (Cabanis and Heine)".

Elainea cinereifrons Salvadori \& Festa, 1899

Salvadori T., Festa E., 1899. Boll. Mus. Zool. An. comp. Univ. Torino, No. 362 (vol. XIV): 8-9. Now: Pseudelaenia leucospodia (Taczanowski, 1877)

HOLOTYPE; MZUT Av1711 - skin; male; Ecuador, Puntilla de S. Elena, 1.1898; collected by E. Festa (field number 2894).

Anaeretes cristatellus Salvadori, 1864

Salvadori T., 1864. Atti Soc. it. Sc. Nat., vol. VII: 153.

Now: Serpophaga subcristata subcristata (Vieillot, 1817)

HOLOTYPE; MZUT Av4972 - dismounted skin; Haiti, no date; obtained from Dr. Ricord in 1843.

Myiobius rufescens Salvadori, 1864

Salvadori T., 1864. Atti Soc. it. Sc. Nat., vol. VII: 152-153.

Now: Myiophobus fasciatus rufescens (Salvadori, 1864) 
HOLOTYPE; MZUT Av4760 - dismounted skin; adult; Brazil? no date; obtained from "Colombi" (from Brest, France).

Notes: "The locality given by Dr. Salvadori [...] is erroneous, the bird being probably restricted in its range to the western coast-region of Peru" (Sclater et Salvin 1868a: 175).

The specimen Av4760 is cited with catalogue number No. 4750 in Salvadori (1864: 152) and in Elter (1986: 315) and, actually, also an old label reads "4750". M. rufescens "may merit treatment as a separate species; see Fitzpatrick (2004)" (Dickinson et Christidis 2014: 55).

\section{Sublegatus frontalis Salvadori, 1897}

Salvadori T., 1897. Boll. Mus. Zool. An. comp. Univ. Torino, No. 292 (vol. XII): 14-15.

Now: Sublegatus modestus brevirostris (d'Orbigny \& Lafresnaye, 1837)

SYNTYPE; MZUT Av1723 - skin; male; Bolivia, Caiza, February 1896; collected by A. Borelli (field number 230);

SYNTYPE; MZUT Av1724 - skin; male; Bolivia, Caiza, March 1896; collected by A. Borelli (field number 305).

Rhynchocyclus cerviniventris Salvadori, 1864

Salvadori T., 1864. Atti Soc. it. Sc. Nat., vol. VII: 153.

Now: Contopus pallidus (Gosse, 1847)

HOLOTYPE; MZUT Av4758 - dismounted skin; "Brazil” (error = Jamaica), no date; obtained from "Colombi" (from Brest, France) in 1846.

Notes: the synonymy was indicated in Sclater et Salvin (1868a: 175 in a note), after the examination of the type specimens of Salvadori, and subsequently it was accepted by Oberholser (1899: 335) and by Salvadori himself (1915: 18). The type locality reported on the MS. catalogue and in Salvadori (1864: 153) is "Brazil (Rio Napo)" but is incorrect, because the range of $C$. pallidus is restricted to Jamaica.

\section{THAMNOPHILIDAE}

Myrmotherula minor Salvadori, 1864

Salvadori T., 1864. Atti Soc. it. Sc. Nat., vol. VII: 157-158.

Now: Myrmotherula minor Salvadori, 1864

HOLOTYPE; MZUT Av2799 - dismounted skin; Brazil, 1840; from A. Caffer coll.

Thamnistes affinis Salvadori, 1864

Salvadori T., 1864. Atti Soc. it. Sc. Nat., vol. VII: 154.

Now: Thamnomanes caesius glaucus Cabanis, 1847

HOLOTYPE; MZUT Av701 - dismounted skin; female; Brazil ("Rio Napo?”), ca. 18301850; unknown origin.

Notes: T. affinis Salvadori $=$ Thamnomanes glaucus Cabanis $q$ (Sclater et Salvin 1868a: 175).

Myrmeciza marginata Salvadori, 1864

Salvadori T., 1864. Atti Soc. it. Sc. Nat., vol. VII: 158-159.

Now: Myrmoderus ruficauda ruficauda (zu Wied, 1831)

SYNTYPE; MZUT Av675 - dismounted skin; female; Brazil? no date; acquired in Paris by F. Bonelli in 1820;

SYNTYPE; MZUT Av4890 (former 4896) - skin; male; Brazil, no date; donated by Carlo Ferreratti in 1847; from Ippolito Blanc collection?

SYNTYPE; MZUT Av4895 - skin; female?; Brazil, no date; donated by Carlo Ferreratti in 1847.

Notes: M. marginata Salvadori = M. ruficauda (Max.) (Sclater et Salvin 1868a: 175). Specimen Av4895 was cited as in the original description (Salvadori 1864: 158).

C. Ferreratti was chief taxidermist of the Turin museum in 1835 (Salvadori 1915: 14). 


\section{MELANOPAREIIDAE}

Synallaxis subspeciosa Salvadori \& Festa, 1899

Salvadori T., Festa E., 1899. Boll. Mus. Zool. An. comp. Univ. Torino, No. 362 (vol. XIV): 21. Now: Melanopareia elegans (Lesson, 1844)

HOLOTYPE; MZUT Av2143 - skin; female; Ecuador, Balzar, December 1897; collected by E. Festa (field number 2719).

\section{GRALLARIIDAE}

Grallaria periophthalmica Salvadori \& Festa, 1898

Salvadori T., Festa E., 1898. Boll. Mus. Zool. An. comp. Univ. Torino, No. 330 (vol. XIII): 2.

Now: Hylopezus perspicillatus periophthalmicus (Salvadori \& Festa, 1898)

HOLOTYPE; MZUT Av12776 - mounted skin; male; Ecuador, Rio Peripa, November 1897; collected by E. Festa (field number 2503).

\section{RHINOCRYPTIDAE}

Acropternis infuscatus Salvadori \& Festa, 1899

Salvadori T., Festa E., 1899. Boll. Mus. Zool. An. comp. Univ. Torino, No. 362 (vol. XIV): 34. Now: Acropternis orthonyx infuscatus Salvadori \& Festa, 1899

SYNTYPE; MZUT Av12774 - skin; male; Ecuador, Pichincha, Lloa, July 1897; collected by C. Ollalla; from E. Festa;

SYNTYPE; MZUT Av15053 (former 2051) - skin; female; Ecuador, Pichincha, Frutillas, June 1897; collected by C. Ollalla (field number 1611); from E. Festa;

SYNTYPE; MZUT Av15054 (former 2052) - skin; male; Ecuador, Pichincha, Lloa, July 1897; collected by C. Ollalla (field number 1696), from E. Festa.

Notes: Salvadori (1915: 38) cites as types only the specimens 2051 and 2052.

Hypocnemis? striativentris Salvadori, 1864

Salvadori T., 1864. Atti Soc. it. Sc. Nat., vol. VII: 159.

Now: Eleoscytalopus indigoticus (zu Wied, 1831)

HOLOTYPE; MZUT Av4954 - skin; Brazil; obtained from sig. Mussino, around 1846?

\section{SCLERURIDAE}

Sclerurus salvini Salvadori \& Festa, 1899

Salvadori T., Festa E., 1899. Boll. Mus. Zool. An. comp. Univ. Torino, No. 362 (vol. XIV): 23-24.

Now: Sclerurus guatemalensis salvini Salvadori \& Festa, 1899

SYNTYPE; MZUT Av2204 - skin; male; Ecuador, Rio Peripa, November 1897; collected by E. Festa (field number 2342);

SYNTYPE; MZUT Av2205 - skin; female; Ecuador, Rio Peripa, November 1897; collected by E. Festa (field number 2416);

SYNTYPE; MZUT Av2206 - skin; female; Ecuador, Rio Peripa, November 1897; collected by E. Festa (field number 2514).

\section{DENDROCOLAPTIDAE}

Dendrocincla brunnea Salvadori \& Festa, 1898

Salvadori T., Festa E., 1898. Boll. Mus. Zool. An. comp. Univ. Torino, No. 330 (vol. XIII): 2. Now: Dendrocincla tyrannina tyrannina (Lafresnaye, 1851)

HOLOTYPE; MZUT Av2272 - skin; female; Ecuador, Nanegal, June 1897; collected by E. Festa (field number 1240).

Dendrocincla macrorhyncha Salvadori \& Festa, 1899

Salvadori T., Festa E., 1899. Boll. Mus. Zool. An. comp. Univ. Torino, No. 362 (vol. XIV): 27.

Now: Dendrocincla tyrannina tyrannina (Lafresnaye, 1851) 
HOLOTYPE; MZUT Av2271 - skin; male; Ecuador, Tulcan, Pun, Huaca, February 1897; collected by E. Festa (field number 745).

Dendrornis erythropygia P. L. Sclater, 1860

Sclater P. L., 1860. Proc. Zool. Soc. London, Part XXVII (III, N. 406): 366 [1859].

Now: Xiphorhynchus erythropygius erythropygius (P. L. Sclater, 1860)

PARALECTOTYPE (possible); MZUT Av6842 - dismounted skin; male; Mexico, near Jalapa (error?), no date; collected by Boucard; donated in 1868.

Notes: on the label of the specimen Av6842 we read “Cotypus!", however neither Salvadori (1915) nor Elter (1986: 181) consider it a type.

In describing Dendrornis erythropygia, Sclater (1860a: 366) indicates the geographical distribution with the expression "In Stat. Veræ Crucis et Oaxaca reipubl. Mexicanæ (Sallé et Boucard)". The type specimens of Sallé, erroneously identified as "Dendrornis triangularis" in Sclater (1857: 289), come from "Cordova" (= Córdoba, Veracruz), and those of Boucard come from Oaxaca (Sclater 1860b: 381). The specimens collected "by Señor Raphael Montes de Oca in the vicinity of Jalapa in the state of Vera Cruz" implicitly form part of the type series, as suggested in the introduction of the work of Sclater (1860a: 362). This interpretation is confirmed by Sclater himself (1890: 132), who in the British Museum's catalogue cites as a "Type of the species" a specimen collected by de Oca in Jalapa, Mexico, and does not report specimens of Sallé or Boucard.

It therefore seems correct to admit that the type series of $D$. erythropygia includes the specimens of de Oca, of Sallé and of Boucard, and that the indication of the "type" made by Sclater (1890: 132) corresponds to a designation of lectotype. The other specimens, including those collected by Sallé and Boucard, therefore correspond to paralectotypes. The specimen of the Turin Museum, acquired in 1868 from Boucard, could be a paralectotype, but it is not certain that it was examined by Sclater: the simple indication of "Cotypus!" on the label cannot represent a sufficient proof.

\section{PTILONORHYNCHIDAE}

Calodera maculata Gould, 1837

Gould J., 1837. Synopsis Birds Australia, Part I, Pl. 6.

Now: Chlamydera maculata (Gould, 1837)

SYNTYPE (possible); MZUT Av3563 - dismounted skin; immature male; Australia; donated by J. Gould in 1842.

Notes: an old label (of J. Gould?) reports: "Chlamydera maculata / New South Wales / a Male"; like all the other specimens received from Gould, Av3563 is treated by Salvadori (1915: 13) and Elter (1986: 132) as a type.

\section{MELIPHAGIDAE}

Ptilotis albonotata Salvadori, 1876

Salvadori T., 1876. Ann. Mus. Civ. Genova, IX: 33.

Now: Meliphaga albonotata (Salvadori, 1876)

SYNTYPE; MZUT Av4111 - skin; adult female; Papua New Guinea, Naiabui, July 1875; collected by L.M. D'Albertis (field number 273).

Ptilotis montana Salvadori, 1880

Salvadori T., 1880. Ann. Mus. Civ. Genova, XVI: 77.

Now: Meliphaga montana montana (Salvadori, 1880)

SYNTYPE; MZUT Av4112 - skin; female; Indonesia, Arfak Mts., 13 May 1875; collected by A.A. Bruijn.

Ptilotis subfrenata Salvadori, 1876

Salvadori T., 1876. Ann. Mus. Civ. Genova, VII: 948-949. 
Now: Caligavis subfrenata subfrenata (Salvadori, 1876)

SYNTYPE; MZUT Av4125 - skin; adult male; Indonesia, West Papua, Hatam, Arfak Mts., 30 June 1875; collected by A.A. Bruijn.

Melidectes torquatus P. L. Sclater, 1874

Sclater P. L., 1874. Proc. Zool. Soc. London, [1873, N. XLIV]: 694-695.

Now: Melidectes torquatus torquatus P.L. Sclater, 1874

SYNTYPE; MZUT Av4100 - skin; adult male; Indonesia, West Papua, Arfak Mts., September 1872; collected by L.M. D'Albertis.

Notes: Sclater (1874: 695) cites a pair of birds obtained by d'Albertis in October 1872, but the month is certainly wrong because the birds were both collected in September.

The second syntype is housed in the Museum of Genoa (Arbocco et al. 1979: 241).

Myzomela pluto Forbes, 1879

T. Salvadori in Forbes, W.A., 1879. Proc. Zool. Soc. London [1879]: 266.

Now: Myzomela nigrita pluto W.A. Forbes, 1879

SYNTYPE; MZUT Av4089 - skin; adult male; Indonesia, W New Guinea, Geelvink Bay, Mios Num ("Miosnom") Is., 30 April 1875; collected by O. Beccari.

Notes: Forbes (1879) did not intend to introduce a new species, as he wrote: "I consider it better to retain all forms under one name". He also reports that "Count Salvadori is inclined to separate them (i.e. the specimens from Jobi and Miosnom) as a new species". Forbes presents a table in which there are, together with others, measurements of the 3 specimens collected by Meyer and Beccari from Jobi and Miosnom, to the side of which he writes "M. pluto, Salvadori, in litt.". We believe that there is reason to attribute the authorship to Salvadori, but so far only Mayr (1941: 194) and Arbocco et al. (1986: 22) seem to have taken this hypothesis into consideration.

Myzomela adolphinae Salvadori, 1876

Salvadori T., 1876. Ann. Mus. Civ. Genova, VII: 946-947.

Now: Myzomela adolphinae Salvadori, 1876

SYNTYPE; MZUT Av4085 - skin; adult male; Indonesia, West Papua, Arfak Mts., June 1874; collected by A.A. Bruijn.

Ptilotis erythropleura Salvadori, 1876

Salvadori T., 1876. Ann. Mus. Civ. Genova, VII: 949-950.

Now: Ptiloprora erythropleura erythropleura (Salvadori, 1876)

SYNTYPE; MZUT Av4127 - skin; male; Indonesia, West Papua, Arfak Mts., 6 May 1875; collected by A.A. Bruijn.

Glyciphila? ocularis Gould, 1838

Gould J., 1838. Synopsis Birds Australia, Part IV, Appendix, p. 6.

Now: Lichmera indistincta ocularis (Gould, 1838)

SYNTYPE (possible); MZUT Av3631 - dismounted skin; Australia, no date; donated by J. Gould in 1842.

Meliphaga sericea Gould, 1837

Gould J., 1837. Synopsis Birds Australia, Part I, Plate 16, Fig. 2.

Now: Phylidonyris niger niger (Bechstein, 1811)

SYNTYPE (possible); MZUT Av3630 - dismounted skin; adult; Australia, no date; donated by J. Gould in 1843.

Haematops validirostris Gould, 1837

Gould J., 1837. Synopsis Birds Australia, Part I, Plate 17, Fig. 2.

Now: Melithreptus validirostris (Gould, 1837)

SYNTYPE (possible); MZUT Av3629 - dismounted skin; adult; Australia, Tasmania, no date; donated by J. Gould in 1842. 


\section{MELANOCHARITIDAE}

Melanocharis chloroptera Salvadori, 1876

Salvadori T., 1876. Ann. Mus. Civ. Genova, VII: 987-988.

Now: Melanocharis nigra chloroptera Salvadori, 1876

SYNTYPE; MZUT Av3953 - skin; male; Indonesia, Aru Is., Wokam, 1873; collected by 0.

Beccari; from Genoa Museum;

SYNTYPE; MZUT Av3954 - skin; female; Indonesia, Aru Is., Wokam, 1873; collected by 0.

Beccari; from Genoa Museum.

Notes: Salvadori $(1915: 30,46)$ reports as a topotype the specimen Av9628, collected by D'Albertis in 1877, but "forgets" the two syntypes collected by O. Beccari; Elter (1986: 291) indicates as type specimens Av3953 and Av3954, but also (and erroneously) considers specimen Av9628 as type.

Melanocharis longicauda Salvadori, 1876

Salvadori T., 1876. Ann. Mus. Civ. Genova, VII: 942-943.

Now: Melanocharis longicauda longicauda Salvadori, 1876

SYNTYPE; MZUT Av3955 - skin; adult male; Indonesia, NW New Guinea, Bird's Head, Arfak Mts., June 1874; collected by A.A. Bruijn; from Genoa Museum;

SYNTYPE; MZUT Av3956 - skin; female; Indonesia, NW New Guinea, Bird's Head, Hatam, 1873; collected by L.M. D’Albertis; from Genoa Museum.

Rhamphocharis crassirostris Salvadori, 1876

Salvadori T., 1876. Ann. Mus. Civ. Genova, VII: 943-944.

Now: Rhamphocharis crassirostris crassirostris Salvadori, 1876

SYNTYPE; MZUT Av3958 - skin; adult male; Indonesia, NW New Guinea, Bird's Head, Hatam, Arfak Mts., 5 July 1875; collected by O. Beccari; from Genoa Museum;

SYNTYPE; MZUT Av3959 - skin; adult female; Indonesia, NW New Guinea, Bird's Head, Hatam, Arfak Mts., 2 July 1875; collected by O. Beccari; from Genoa Museum.

Melilestes iliolophus Salvadori, 1876

Salvadori T., 1876. Ann. Mus. Civ. Genova, VII: 951-952.

Now: Oedistoma iliolophus iliolophus (Salvadori, 1876)

SYNTYPE; MZUT Av4097 - skin; male; Indonesia, New Guinea, Japen Is., 6 April 1875; collected by 0 . Beccari.

\section{CAMPEPHAGIDAE}

Pericrocotus griseogularis Gould, 1863

Gould J., 1863. Proc. Zool. Soc. London, Part XXX [1862], n. XVIII: 282.

Now: Pericrocotus solaris griseogularis Gould, 1863

SYNTYPE (possible); MZUT Av5562; skin, male; Taiwan, April 1862; collected by Swinhoe; from E. Giglioli.

Notes: Elter (1986: 352) considers the specimen Av5562 as a type of P. griseigularis (sic) but Salvadori (1915) does not report any type of this species in the Turin Museum.

Two syntypes, hosted in the National Museum of Natural History, Smithsonian Institution, Washington (former United States National Museum, USNM), come from Swinhoe and were both captured in April 1862 (http://collections.nmnh.si.edu/search/birds/ accessed 16 July 2017). These data coincide with those of the specimen Av5562, however it is possible that Gould has never seen them, because they may have been donated directly from Swinhoe to E.H. Giglioli. According to Dickinson et al. (2002: 43) the only known types would be those of the USNM collection.

Pericrocotus modiglianii Salvadori, 1892

Salvadori T., 1892. Ann. Mus. Civ. Genova, XXXII: 130-133.

Now: Pericrocotus flammeus modiglianii Salvadori, 1892

SYNTYPE; MZUT Av11446 - skin; adult male; Indonesia, Enggano Is., Pulau, Kifa-juc, 8 
May 1891; collected by E. Modigliani (field number 38);

SYNTYPE; MZUT Av11447 - skin; adult female; Indonesia, Enggano Is., Pulau, Kifa-juc, 8 May 1891; collected by E. Modigliani (field number 39).

Notes: Dickinson et al. (2002: 44) report only the occurrence of type specimens in the Museum of Genoa (8 syntypes quoted by Arbocco et al. 1979: 212).

Graucalus pollens Salvadori, 1874

Salvadori T., 1874. Ann. Mus. Civ. Genova, VI: 75-77.

Now: Coracina personata pollens (Salvadori, 1874)

SYNTYPE; MZUT Av3098 - skin; male; Indonesia, Kai Is., December 1872; collected by L.M. D’Albertis;

SYNTYPE; MZUT Av3099 - skin; female; Indonesia, 'Kei Ralan', 2 October 1873; collected by 0 . Beccari.

Notes: Dickinson et al. (2002: 34) report only the occurrence of type specimens in the Museum of Genoa (3 syntypes quoted by Arbocco et al. 1979: 209).

Graucalus enganensis Salvadori, 1892

Salvadori T., 1892. Ann. Mus. Civ. Genova, XXXII: 129-130.

Now: Coracina striata enganensis (Salvadori, 1892)

SYNTYPE; MZUT Av3100 - skin; “male?”; Indonesia, Enggano Is., Bua Bua, 21 May 1891; collected by E. Modigliani (field number 106);

SYNTYPE; MZUT Av11445 - skin; female; Indonesia, Enggano Is., Kifa-juc, 6 May 1891; collected by E. Modigliani (field number 12).

Notes: Salvadori (1915: 36) among the types of Graucalus enganensis does not list the specimen Av3100 but reports Av5930, which is actually a syntype of Gracula enganensis Salvadori (see below). Elter (1986: 226) also reports the specimen Av5930 as a type of Graucalus enganensis.

Dickinson et al. (2002: 35) report only the occurrence of type specimens in the Museum of Genoa (5 syntypes quoted by Arbocco et al. 1979: 209).

Edoliisoma nehrkorni Salvadori, 1890

Salvadori T., 1890. Aggiunte alla Ornitologia della Papuasia e delle Molucche. Parte seconda - Passeres. Torino, Clausen, p. 91.

Now: Edolisoma tenuirostre nehrkorni Salvadori, 1890

HOLOTYPE; MZUT Av10362 - skin; male; New Guinea, Waigeo Is., 11 February 1884; collected by Dr. Platen and donated by A. Nehrkorn.

Notes: Av10362 is a specimen quoted by Nehrkorn (1885: 32) as Edoliisoma remotum, but Salvadori (1886: 152) already suspected that the identification was not correct.

The Aggiunte alla Ornitologia della Papuasia e delle Molucche - parte seconda (Salvadori, 1890a) are a preprint of the same work of Salvadori (1890b) published in the Memorie della R. Accademia delle Scienze di Torino, although they do not advance the date of publication (Dickinson 2011b). In the volume of the Memorie, Edoliisoma nehrkorni is described on p. 217.

\section{CINCLOSOMATIDAE}

Eupetes nigricrissus Salvadori, 1876

Salvadori T., 1876. Ann. Mus. Civ. Genova, IX: 36-37.

Now: Ptilorrhoa caerulescens nigricrissus (Salvadori, 1876)

SYNTYPE; MZUT Av4338 - skin; adult, male?; New Guinea, Amama river, Naiabui, September 1875; collected by L.M. D’Albertis (field number 452).

\section{PACHYCEPHALIDAE}

Pachycephala cinerascens Salvadori, 1878

Salvadori T., 1878. Ann. Mus. Civ. Genova, XII: 332. 
Now: Pachycephala griseonota cinerascens Salvadori, 1878

SYNTYPE; MZUT Av3371 - skin; Indonesia, Is. Molucche, Ternate, 26 November 1874; collected by 0 . Beccari.

\section{ORIOLIDAE}

Rectes decipiens Salvadori, 1878

Salvadori T., 1878. Ann. Mus. Civ. Genova, XII: 473.

Now: Pitohui kirhocephalus decipiens (Salvadori, 1878)

SYNTYPE; MZUT Av3322 - skin; male; Indonesia, Kapaur, W Nuova Guinea, 1872; collected by L.M. D'Albertis (field number 24).

Notes: type-localities are clearly indicated in the bibliography of $R$. decipiens and in the text that precedes the description (Salvadori 1878e: 471-473): Kapaur / Kapaor, Rubi, Waweji, Inviorage, Nappan, Mum, Kulukadi, Warbusi. However, Paynter (1967: 46) wrote "no locality; Kapaur designated by Stresemann, 1923".

At the Museum of Genoa there are other 4 syntypes from Kapaur, Kulukadi and Warbusi (Arbocco et al. 1979: 230).

\section{RHAGOLOGIDAE}

Pachycephala leucostigma Salvadori, 1876

Salvadori T., 1876. Ann. Mus. Civ. Genova, VII: 933-934.

Now: Rhagologus leucostigma leucostigma (Salvadori, 1876)

SYNTYPE; MZUT Av3374 - skin; female; Indonesia, NW New Guinea, Bird's Head, Hatam, July 1875; collected by 0 . Beccari.

Notes: Av3374 corresponds to the specimen "d" in Salvadori (1881b: 234).

\section{ARTAMIDAE}

Vanga nigrogularis Gould, 1837

Gould J., 1837. Synopsis Birds Australia, Part I, Plate 3, Fig. 2 and text.

Now: Cracticus nigrogularis nigrogularis (Gould, 1837)

SYNTYPE (possible); MZUT Av3546 - skin; adult; Australia, "New Holland", no date; donated by J. Gould in 1842 .

Notes: the specimen Av3546 is quoted as a type of Cracticus nigrigularis (sic) by Salvadori (1915: 13) and Elter (1986: 164).

\section{VANGIDAE}

Muscicapa hirundinacea Temminck, 1822

Temminck in Temminck C.-J. \& Laugier de Chartrouse M., 1822. Planches Coloriées, livr. 20, pl. 119.

Now: Hemipus hirundinaceus (Temminck, 1822)

SYNTYPE (possible); MZUT Av926 - dismounted skin; Indonesia, Java, no date; collected by H. Kuhl \& J. C. van Hasselt (1820-21); donated by Temminck in 1827 (sic in MS. Catalogue),

SYNTYPE (possible); MZUT Av927 - dismounted skin; Indonesia, Java, no date; collected by H. Kuhl \& J. C. van Hasselt (1820-21); donated by Temminck in 1826.

Notes: "Muscicapa hirundinacea Reinw." in the MS. Catalogue of F. A. Bonelli (NN. 3599, 3655). Salvadori (1915: 7) and Elter (1986: 312) consider these specimens as types. Temminck et Laugier (1822a) did not indicate the origin of the specimens described but attributed the authorship of M. hirundinacea to Carl Reinwardt. Not even the origin of the two syntypes housed in the Museum of Leiden is known but they belonged to the old collection (Dekker 2003: 26). 


\section{MALACONOTIDAE}

Malaconotus poliocephalus shoanus Neumann, 1903

Neumann O., 1903. Ornithologische Monatsberichte, XI (9): 89.

Now: Malaconotus blanchoti approximans (Cabanis, 1869)

HOLOTYPE; MZUT Av3259; skin; male; Etiopia, S Shewa, Ambukarra, 22 August 1878; collected by 0 . Antinori (field number 641).

Notes: specimen Av3259 was misidentified as Meristes hypopyrrhus by Salvadori (1884: 134).

\section{DICRURIDAE}

Buchanga periophthalmica Salvadori, 1894

Salvadori T., 1894. Ann. Mus. Civ. Genova, XXXIV: 594.

Now: Dicrurus leucophaeus periophthalmicus (Salvadori, 1894)

SYNTYPE; MZUT Av11464 - dismounted skin; adult; Indonesia, Mentawei, Si Oban or Si Pora, no date; collected by E. Modigliani.

Dicruropsis viridinitens Salvadori, 1894

Salvadori T., 1894. Ann. Mus. Civ. Genova, XXXIV: 593-594.

Now: Dicrurus hottentottus viridinitens (Salvadori, 1894)

SYNTYPE; MZUT Av11463 - skin; adult male; Indonesia, Mentawei, Si Oban, 11 May 1894; collected by E. Modigliani (field number 193).

\section{RHIPIDURIDAE}

Rhipidura atra Salvadori, 1876

Salvadori T., 1876. Ann. Mus. Civ. Genova, VII: 922-923.

Now: Rhipidura atra atra Salvadori, 1876

SYNTYPE, MZUT Av2975; skin; male; Indonesia, NW New Guinea, Vogelkopf, Hatam (Arfak), 29.6.1875; collected by A.A. Bruijn; from Genoa Museum.

Notes: specimen Av2975 is not quoted in Salvadori (1915), however it corresponds to specimen "f" of the type list of $R$. atra in Salvadori (1881b: 72).

\section{LANIIDAE}

\section{Lanius souzae Bocage, 1878}

Bocage J. V. Barboza du, 1878. Jornal Sciencias Math. Phys. e Nat., Num. XXIII: 208-214.

Now: Lanius souzae souzae Bocage, 1878

SYNTYPE (possible); MZUT Av9432 - skin; female; Angola, no date; collected by d'Anquieta; from Lisbon Museum in 1879.

Notes: Salvadori (1915) does not cite this specimen as a type, nor does Elter (1986: 265). However, specimen Av9432 was collected by d'Anquieta, so it could be one of four specimens listed by Bocage (1878: 213). From "Museus da Universidade de Lisboa" it was not possible to obtain information on birds collected by d'Anquieta, because "the Museu Bocage's former zoological collection was lost during a huge fire in 1978. All Bird Collection was lost, including the type-specimens" (J. Alves pers. comm. to G. Aimassi, 22 March 2017).

Lanius dealbatus De Filippi, 1853

De Filippi F., 1853. Rév. et Mag. Zool. pure appl., 2e sér., t. V: 289.

Now: Lanius excubitor leucopygos Hemprich \& Ehrenberg, 1833

LECTOTYPE; MZUT Av1509 - dismounted skin; adult; Sudan, White Nile, from $4^{\circ}$ and $3^{\circ}$ lat. N; collected by A. Brun-Rollet on an unspecified date and sent to the Turin Museum in 1853. 
PARALECTOTYPE; MZUT Av737 - dismounted skin; adult; Libya, Tripoli, 1819; collected by Leach; from A. Bonelli.

Notes: Salvadori (1868b: 277), according to De Filippi (1853: 290), reports both specimens as types. Subsequently Salvadori himself (1915: 15) indicates as "type" the specimen n. 1509, explicitly excluding the n. 737, which he identifies as L. hemileucurus (now L. excubitor elegans Swainson, 1832, cf. Mayr et Greenway 1960: 358, footnote 1). This statement can be considered as a designation of lectotype (Aimassi et Ghiraldi 2017: 68).

Lanius pallidus Antinori, 1864

Antinori 0., 1864. Catalogo descrittivo di una collezione di uccelli fatta da Orazio Antinori nell'interno dell'Affrica centrale nord dal maggio 1859 al luglio 1861 . Milano, 117 pp; p. 56.

Now: Lanius excubitor pallidirostris Cassin, 1851

HOLOTYPE; MZUT Av5545 - dismounted skin; adult male; Sudan, Kadaref, November 1859; collected by 0 . Antinori (field number 287).

Lanius macrocercus De Filippi, 1853

De Filippi F., 1853. Rév. et Mag. Zool. pure appl., 2e sér., t. V: 290.

Now: Lanius excubitoroides excubitoroides Prévost \& De Murs, 1851

SYNTYPE; MZUT Av1480 - dismounted skin; adult female; Sudan, White Nile, from $4^{\circ}$ and $3^{\circ}$ lat. N; collected by A. Brun-Rollet on an unspecified date and sent to the Turin Museum in 1853;

SYNTYPE; MZUT Av1687 - dismounted skin; adult male; Sudan, White Nile, from $4^{\circ}$ and $3^{\circ}$ lat. $\mathrm{N}$; collected by A. Brun-Rollet on an unspecified date and sent to the Turin Museum in 1853;

SYNTYPE; MZUT Av5833 - dismounted skin; adult female; Sudan, White Nile, from $4^{\circ}$ and $3^{\circ}$ lat. N; collected by A. Brun-Rollet on an unspecified date and sent to the Turin Museum in 1853;

SYNTYPE (possible); MZUT Av3205 - skin; adult male; Sudan, White Nile, from $4^{\circ}$ and $3^{\circ}$ lat. N; collected by A. Brun-Rollet on an unspecified date and sent to the Turin Museum in 1853.

Notes: Salvadori (1868b: 279) omits specimen Av3205 from the type series; Salvadori (1915: 15) omits specimens Av3205 and Av5833. The latitude is indicated by De Filippi (1853: 289) and Salvadori (1915: 15).

Lanius antinorii Salvadori, 1878

Salvadori T., 1878. Ann. Mus. Civ. Genova, XII: 316.

Now: Lanius somalicus Hartlaub, 1859

HOLOTYPE; MZUT Av10517 - dismounted skin; adult; Ethiopia, Danakil, Mullu, 16 August 1876; collected by 0 . Antinori (field number 27).

Lanius pyrrhostictus Holub \& Pelzeln, 1882

Holub E., Pelzeln A., 1882. Beiträge Ornithologie Südafrikas, Wien, pp. 97-98, pl. 2.

Now: Lanius collaris subcoronatus A. Smith, 1841

PARATYPE; MZUT Av12276 - skin; adult female; South Africa, Marioc Distr., Lino Kane, no date; collected by E. Holub.

Notes: Salvadori $(1915: 40,45)$ considers the Av12276 as a type, but the original paper of Holub et Pelzeln (1882: 97-98) does not confirm this hypothesis.

\section{CORVIDAE}

Cissa hypoleuca Salvadori \& Giglioli, 1885

Salvadori T., Giglioli E. H., 1885. Atti R. Acc. Sc. Torino, vol. XX: 343-344.

Now: Cissa hypoleuca hypoleuca Salvadori \& Giglioli, 1885

HOLOTYPE; MZUT Av6087 - skin; Vietnam, “Thu-Dan-Mot”, June 1866; from E.H. Giglioli 
\& F. De Filippi (Magenta voyage).

Notes: Salvadori (1915: 21) quotes this specimen with the wrong number 6802.

Garrulus melanocephalus Gené, 1834

Gené G., 1834. Mem. R. Acc. Sc. Torino, T. XXXVII: 293-295, Pl. 1.

Now: Garrulus glandarius atricapillus I. Geoffroy Saint-Hilaire, 1832

SYNTYPE; MZUT Av333 - dismounted skin; immature; Lebanon, Mount Lebanon or Baalbek, January 1830; from Crolla.

SYNTYPE; MZUT Av334 - dismounted skin; adult; Lebanon, Mount Lebanon or Baalbek, June 1830; from Crolla.

Notes: Salvadori (1915: 9-10) and Elter (1986: 217) quote the two syntypes with the numbers of the old MS. Catalogue (4092 and 4103), in which they are named "Corvus melanocephalus Bonelli".

Garrulus rhodius Salvadori \& Festa, 1913

Salvadori T., Festa E., 1913. Boll. Mus. Zool. An. comp. Univ. Torino, No. 673 (vol. XXVIII): 2-3.

Now: Garrulus glandarius anatoliae Seebohm, 1883

SYNTYPE; MZUT Av7522 - skin; adult female; Greece, Rhodes, Ativiros Mt., 2 May 1913; collected by E. Festa (field number 733);

SYNTYPE; MZUT Av7523 - skin; adult; Greece, Rhodes, Ativiros Mt., 5 May 1913; collected by E. Festa;

SYNTYPE; MZUT Av7524 - skin; adult male; Greece, Rhodes, Ativiros Mt., 7 May 1913; collected by E. Festa (field number 267);

SYNTYPE; MZUT Av7525 - skin; adult male; Greece, Rhodes, Ativiros Mt., 7 May 1913; collected by E. Festa (field number 273).

Cyanolyca angelae Salvadori \& Festa, 1899

Salvadori T., Festa E., 1899. Boll. Mus. Zool. An. comp. Univ. Torino, No. 357 (vol. XIV): 30-31.

Now: Cyanolyca armillata quindiuna Sclater \& Salvin, 1876

SYNTYPE; MZUT Av6162 - skin; female; Ecuador, Tulcan, Pun, Huaca, February 1897; collected by E. Festa (field number 669);

SYNTYPE; MZUT Av6163 - skin; male; Ecuador, Tulcan, Pun, Huaca, February 1897; collected by E. Festa (field number 704).

\section{MONARCHIDAE}

Monarcha aruensis Salvadori, 1874

Salvadori T., 1874. Ann. Mus. Civ. Genova, VI: 309-310.

Now: Carterornis chrysomela aruensis (Salvadori, 1874)

SYNTYPE; MZUT Av3063 - skin; adult male; Indonesia, Aru Is., Giabu-lengan, 20 May 1873; collected by 0 . Beccari (field number 381);

SYNTYPE; MZUT Av15862 - skin; adult male; Indonesia, Aru Is., Wokan, 28 May 1873; collected by 0 . Beccari (field number 425);

SYNTYPE; MZUT Av15863 - skin; adult female; Indonesia, Aru Is., 1873; collected by 0. Beccari.

Notes: it is not clear why Elter (1986: 367) and Salvadori (1915) did not consider these specimens as types. The type series cited by Salvadori (1874e: 310 ) includes 6 males and 2 females collected in the Aru Islands by 0 . Beccari, which correspond to the 8 specimens cited by Salvadori (1881b: 40-41) with the letters a-h. The specimens Av3063, Av15862 and Av15863 correspond, respectively, to the specimens $\mathrm{f}, \mathrm{b}, \mathrm{h}$.

Four other syntypes are housed in the Genoa Museum (Arbocco et al. 1979: 228).

Terpsiphone insularis Salvadori, 1887

Salvadori T., 1887. Ann. Mus. Civ. Genova, XXIV: 539-540. 
Now: Terpsiphone affinis insularis Salvadori, 1887

SYNTYPE; MZUT Av10615 - mounted skin; male, Indonesia, Nias Is., 5 August 1886; collected by E. Modigliani (field number 190);

SYNTYPE; MZUT Av10616 - mounted skin; female; Indonesia, Nias Is., 28 May 1886; collected by E. Modigliani (field number 82).

Notes: the specimens Av10615 and Av10616 correspond to the specimens "e" and "a" cited by Salvadori (1887b: 540). Two other syntypes in the Genoa Museum (Arbocco et al. 1979: 228).

\section{CORCORACIDAE}

Struthidea cinerea Gould, 1837

Gould J., 1837. Synopsis Birds Australia, Part I, Plate 9 and text.

Now: Struthidea cinerea cinerea Gould, 1837

SYNTYPE (possible); MZUT Av3562 - dismounted skin; Australia, New South Wales, Port Essington, no date; donated by J. Gould in 1842.

\section{PARADISAEIDAE}

Paradisea novae guineae D’Albertis \& Salvadori, 1879

D’Albertis L. M., Salvadori T., 1879. Ann. Mus. Civ. Genova, XIV: 96.

Now: Paradisaea apoda novaeguineae D’Albertis \& Salvadori, 1879

SYNTYPE; MZUT Av9634 - dismounted skin; adult male; Papua New Guinea, Fly River, July 1876; collected by L.M. D’Albertis (field number 67); from G. Doria.

Paradisaea raggiana P. L. Sclater, 1873

Sclater P. L., 1873. Proc. Zool. Soc. London, XXXV: 559.

Now: Paradisaea raggiana raggiana P. L. Sclater, 1873

SYNTYPE; MZUT Av6064 - skin; adult male; Australia, Orangerie Bay, 1873; collected by L.M. D'Albertis.

Notes: Salvadori (1915: 29) also considers the specimen Av9635 as a type, but the capture site (Fly river) was not mentioned by Sclater. In fact, the second syntype is housed in the Genoa Museum (Arbocco et al. 1979: 248).

\section{PETROICIDAE}

Leucophantes hypoxanthus Salvadori, 1876

Salvadori T., 1876. Ann. Mus. Civ. Genova, VII [1875]: 920.

Now: Devioeca papuana (A. B. Meyer, 1875)

SYNTYPE; MZUT Av2883 - skin; male? Indonesia, NW New Guinea, Arfak (Laki?), 11 May 1875; collected by A.A. Bruijn.

Notes: the specimen Av2883 is not reported as a type in Salvadori (1915) and in Elter (1986: 303), but certainly agrees with the "h" specimen cited among the eight types in Salvadori (1881b: 92), which however reported erroneously the year 1873.

We can read the exact capture data on an old label: on the opposite sides we read "Arfak / 11.5.75 / § Laki q" and "Leucophantes hypoxanthus Salvadori nov. sp. / Typus / Bruijn". A second label by Salvadori already indicates the name "Poecilodryas papuana (Meyer)".

Monachella saxicolina Salvadori, 1874

Salvadori T., 1874. Ann. Mus. Civ. Genova, VI: 83.

Now: Monachella muelleriana (Schlegel, 1871)

SYNTYPE; MZUT Av2933 - skin; unsexed; Indonesia, Bird's Head, NW New Guinea, Andai, 1872; collected by D'Albertis;

SYNTYPE; MZUT Av2934 - skin; unsexed; Indonesia, Bird's Head, NW New Guinea, Hatam, 1872; collected by D’Albertis. 
Notes: the description of Salvadori (1874c: 83) was based on five specimens collected by D'Albertis at "Atam", however some years later Salvadori (1881b: 83) argued that the "types" would be only three, listed as "a" (= Av2933) and "b-c" (c = Av2934). The original labels states that both specimens of Turin are types and that they were originally preserved in "spirit (alcohol) of wine".

Since 3 syntypes are housed in the Museum of Genoa (Arbocco et al. 1979: 224), the total of the types of Turin and Genoa agrees with the number of specimens originally described.

\section{DICAEIDAE}

Prionochilus xanthopygius Salvadori, 1868

Salvadori T., 1868. Atti R. Acc. Sc. Torino, vol. III: 416-417.

Now: Prionochilus xanthopygius Salvadori, 1868

SYNTYPE; MZUT Av8227 - dismounted skin; male; Malaysia, Borneo, Sarawak, August 1866; collected by O. Beccari (field number 735); from G. Doria;

SYNTYPE; MZUT Av8228 - dismounted skin; male; Malaysia, Borneo, Sarawak, August 1866; collected by O. Beccari (field number 738); from G. Doria.

Notes: two syntypes, $\hat{O}$ and $q$ captured in Sarawak on 12 December 1865 by G. Doria, are kept in the Museum of Genoa (Arbocco et al. 1979: 232-233).

Dicaeum nigrimentum Salvadori, 1874

Salvadori T., 1874. Ann. Mus. Civ. Genova, V: 165-166.

Now: Dicaeum cruentatum nigrimentum Salvadori, 1874

SYNTYPE; MZUT Av6235 - dismounted skin; male; Malaysia, Borneo, Sarawak, September 1865; collected by G. Doria (field number 259).

Dicaeum sanghirense Salvadori, 1876

Salvadori T., 1876. Ann. Mus. Civ. Genova, IX: 58-59.

Now: Dicaeum celebicum sanghirense Salvadori, 1876

SYNTYPE; MZUT Av3939 - skin; male; Indonesia, N Sulawesi, Sanghir Is., Petà, September 1875; collected by A.A. Bruijn; from Genoa Museum.

Notes: Arbocco et al. (1979: 234) report the presence of 6 syntypes in the Genoa Museum (the type series includes 7 specimens).

Dicaeum keiense Salvadori, 1874

Salvadori T., 1874. Ann. Mus. Civ. Genova, VI: 313-314.

Now: Dicaeum hirundinaceum keiense Salvadori, 1874

SYNTYPE; MZUT Av3937 - skin; adult male; Indonesia, Grande Kai Is., Weri, 5 August 1873; collected by 0. Beccari (field number 619).

Notes: the second syntype is housed in the Genoa Museum (Arbocco et al. 1979: 234).

Dicaeum misoriense Salvadori, 1876

Salvadori T., 1876. Ann. Mus. Civ. Genova, VII: 945.

Now: Dicaeum geelvinkianum misoriense Salvadori, 1876

SYNTYPE; MZUT Av3943 - skin; adult male; N New Guinea, Korido, Misori, 9 May 1875; collected by 0 . Beccari; from Genoa Museum;

SYNTYPE; MZUT Av3944 - skin; female; N New Guinea, Korido, Misori, 8 May 1875; collected by 0 . Beccari; from Genoa Museum.

\section{NECTARINIIDAE}

Nectarinia inornata Temminck, 1821

Temminck in Temminck C.-J. \& Laugier de Chartrouse M., 1821. Planches Coloriées, livr. 14, pl. 84, fig. 2.

Now: Arachnothera affinis (Horsfield, 1821) 
SYNTYPE (possible); MZUT Av2219 - skin; adult male; Indonesia, Java, no date; collected by H. Kuhl and J.C. van Hasselt (1820-21); from Temminck (Leiden Museum) in 1826.

Eleocerthia ragazzii Salvadori, 1888

Salvadori T., 1888. Ann. Mus. Civ. Genova, XXVI: 247.

Now: Cyanomitra olivacea ragazzii (Salvadori, 1888)

SYNTYPE; MZUT Av4068 - skin; adult male; Ethiopia, Fekeriè-Ghemb, 5 May 1885; collected by V. Ragazzi (field number 147);

SYNTYPE; MZUT Av4069 - skin; adult female; Ethiopia, Fekeriè-Ghemb, 28 June 1886; collected by V. Ragazzi (field number 687).

Notes: specimen Av4068 was indicated as female on the original label.

Nectarinia acik Hartmann, 1866

Hartmann R., 1866. Journal für Ornithologie, XIV: 205-207.

Now: Chalcomitra senegalensis acik (Hartmann, 1866)

SYNTYPE; MZUT Av5576; dismounted skin; immature male ["in moult" on the label]; Sudan, Bahr-el-Gazal Province, no date; collected by 0. Antinori (field number 121);

SYNTYPE; MZUT Av5577; dismounted skin; female; C Africa, no date; collected by 0. Antinori (field number 53);

SYNTYPE; MZUT Av5763; dismounted skin; adult male; Sudan, Bahr-el-Gazal Province, no date; collected by 0 . Antinori (field number 47);

SYNTYPE; MZUT Av5842; dismounted skin; immature male; Sudan, Bahr-el-Gazal Province, no date; collected by 0 . Antinori (field number 48).

Notes: Salvadori $(1870: 731 ; 1915: 18)$ claims that the description of $N$. acik was written by Orazio Antinori (in Hartmann 1866: 205), and that was based on the specimens that in the Italian edition of his "Catalogo" (Antinori 1864: 33) had been identified as $N$. natalensis.

Chalcomitra scioana Salvadori, 1888

Salvadori T., 1888. Ann. Mus. Civ. Genova, XXVI: 247-248.

Now: Chalcomitra senegalensis proteus (Rüppell, 1840)

SYNTYPE; MZUT Av4059 - skin; adult male; Ethiopia, Ankober, Mehal Wenz, 1 July 1886; collected by V. Ragazzi (field number 695);

SYNTYPE; MZUT Av4060 - skin; male; Ethiopia, Ankober, Mehal Wenz, 6 July 1886; collected by V. Ragazzi (field number 727);

SYNTYPE; MZUT Av4061 - skin; male; Ethiopia, Zwai Lake, June 1886; collected by V. Ragazzi (field number 757);

SYNTYPE; MZUT Av4062 - skin; female; Ethiopia, Ankober, Mehal Wenz, 6 July 1886; collected by V. Ragazzi (field number 729);

SYNTYPE; MZUT Av10629 - skin; M; Ethiopia, Sciotalit, 24 June 1886; collected by V. Ragazzi (field number 675); from Società Geografica Italiana.

SYNTYPE; MZUT Av10630 - dismounted skin; adult male; Ethiopia, Duleccia river, 22 May 1878; collected by 0 . Antinori.

Notes: Salvadori (1915: 34) lists only three "types", indicating the number of the Ragazzi collection and not that of the Museum catalogue: 695 (= Av4059), 727 (Av4060) and 729 (Av4062). However, in the original description Salvadori (1888b: 248) lists the five specimens above mentioned.

The specimen Av10630, collected by 0. Antinori and already reported as type by Elter (1986: 127), corresponds to the specimen "a" of the seven birds that Salvadori (1884: 141) had misidentified as "Chalcomitra cruentata (Rüpp.)", to which he made a bibliographic reference in the description of the new species (Salvadori 1888b: 247). According to the Art. 72.4.2 of the Code (ICZN 1999) it should be included in the type series.

Cinnyris proteus Rüppell, 1840

Rüppell E., 1840. Neue Wirbelthiere zu der Fauna von Abyssinien gehörig. Vögel, pag. 91. 
Now: Chalcomitra senegalensis proteus (Rüppell, 1840)

SYNTYPE; MZUT Av2207 - dismounted skin; adult male; Ethiopia, Abyssinia, 20 November 1832; collected by E. Rüppell (field number 233).

Notes: specimen collected by Rüppell in Abyssinia in 1832 and sent to the Turin Museum in 1839; it was referred to as "Cotipo" by Salvadori (1915: 10-11).

Rüppell collected bird specimens in Africa in 1822-1827 and in 1831-1834 (Steinheimer, 2005b: 165). He gave all bird specimens MS. names, so most of the new names were already on the skins when they arrived at Frankfurt. We know from Leiden and Tring museums that these specimens were forwarded with the MS. names attached. The specimen Av2207 is therefore to be considered a Syntype (F. D. Steinheimer, pers. comm. to G. Aimassi, 1 October 2015).

Chalcostetha chlorolaema Salvadori, 1874

Salvadori T., 1874. Ann. Mus. Civ. Genova, VI: 77.

Hermotimia theresia Salvadori, 1874 (1875?)

Salvadori T., 1874 (1875?). Atti R. Acc. Sc. Torino, vol. X: 214-216.

Now: Leptocoma aspasia chlorolaema (Salvadori, 1874)

SYNTYPE; MZUT Av4013 - skin; adult male; Indonesia, Kei Ralan, 28 September 1873; collected by 0 . Beccari.

Notes: specimen Av4013 is a syntype of both species: H. theresia is a junior objective synonym of $C$. chlorolaema. Salvadori (1874f: 216; see also Paynter 1967: 240) introduced $H$. theresia as a new name for $C$. chlorolaema because he believed it was preoccupied by "Nectarinia chlorolaema Jardine", cited in Hartlaub et Monteiro (1860: 110). Actually, Jardine never introduced this name, nor did Hartlaub \& Monteiro, who did not provide description or valid indication.

Poggi (2010: 343) reports as "IX-XI.1874" the date of publication of Salvadori's work in the Ann. Mus. Civ. Genova. The paper of Salvadori published in Atti R. Acc. Sc. Torino is certainly subsequent, because it was presented during the session of 13 December 1874. Therefore, it is very likely that the publication took place in 1875.

Hermotimia nigriscapularis Salvadori, 1876

Salvadori T., 1876. Ann. Mus. Civ. Genova, VII: 937-939.

Now: Leptocoma aspasia nigriscapularis (Salvadori, 1876)

SYNTYPE; MZUT Av4012 - skin; adult male; Indonesia, New Guinea, Geelwink Bay, Misonom Is., 30 April 1875; collected by 0. Beccari.

Hermotimia cornelia Salvadori, 1878

Salvadori T., 1878. Atti R. Acc. Sc. Torino, vol. XIII: 319-321.

Now: Leptocoma aspasia cornelia (Salvadori, 1878)

SYNTYPE; MZUT Av9374 - dismounted skin; adult male; Indonesia, New Guinea, Tarawai Is., 1878; collected by Laglaize; from A.A. Bruijn.

SYNTYPE; MZUT Av9375 - dismounted skin; adult male; Indonesia, New Guinea, Tarawai Is., 1878; collected by Laglaize; from A.A. Bruijn;

SYNTYPE; MZUT Av9376 - dismounted skin; female; Indonesia, New Guinea, Tarawai Is., 1878; collected by Laglaize; from A.A. Bruijn.

Nectarinia cupreonitens Shelley, 1876

Shelley G. E., 1876. A Monograph of The Nectariniidae: 17-18.

Now: Nectarinia famosa cupreonitens Shelley, 1876

SYNTYPE; MZUT Av2216 - Ethiopia, Abyssinia, 17.7.1832; collected by E. Rüppell.

Notes: Salvadori (1915: 11) and Elter (1986: 143) consider this specimen, sent to the Turin Museum by Rüppell in 1839 and labelled "Cinnyris famosa (Cuv.) / Abyssinien”, as a type of the taxon Cinnyris famosa Rüppell. However, this is incorrect, because Rüppell (1840: 90) merely quotes $C$. famosus (Cuv.), noting that it is "very common in the province 
of Simen in Abyssinia". Therefore "Cinnyris famosa Rüppell" is not an original description according to the Code (ICZN 1999).

Nonetheless, according to Shelley (1876: 17) the specimens of $C$. famosa Rüppell (nec Cuvier, neque Linnaeus) belong to a new taxon, which he names Nectarinia cupreonitens. His description is primarily based on a male housed at the British Museum (now Tring) once belonging to Sharpe (Warren et Harrison 1971: 138 indicate it as the holotype). Shelley also refers to the description of a female published by Finsch and Hartlaub (1870: 213), which is based on a Rüppell specimen from Abyssinia, hosted in the Bremen Museum (Sànchez-Osés 2010 does not mention it). Furthermore, Shelley lists a synonymy which refers in its first line to the individuals that Rüppell (1840) misidentified as "Cinnyris famosus (Cuv.)".

We believe that the specimen Av2216 could be considered a syntype of Nectarinia cupreonitens Shelley, according to the Art. 72.4.2 (ICZN 1999), because it was originally part of Rüppell's batch used for his 1840 publication.

Nectarinia subfamosa Salvadori, 1884

Salvadori T., 1884. Ann. Mus. Civ. Genova, Serie 2a , Vol. 1 (XXI): 138.

Now: Nectarinia famosa cupreonitens Shelley, 1876

HOLOTYPE; MZUT Av3973 - skin; adult male; Ethiopia, Antotto, December 1881; collected by 0 . Antinori.

Notes: in Salvadori (1915: 32) quoted with number 3913 (error).

Nectarinia Gonzenbachii Antinori, 1864

Antinori 0., 1864. Catalogo descrittivo di una collezione di uccelli fatta da Orazio Antinori nell'interno dell'Affrica centrale nord dal maggio 1859 al luglio 1861. Milano, pp. 35-36.

Now: Cinnyris erythrocercus (Hartlaub, 1857)

HOLOTYPE; MZUT Av5578 - dismounted skin; adult male; Sudan, White Nile, 18601861; collected by 0 . Antinori.

\section{PLOCEIDAE}

Textor scioanus Salvadori, 1884

Salvadori T., 1884. Ann. Mus. Civ. Genova, XXI: 195-196.

Now: Bubalornis niger intermedius (Cabanis, 1868)

SYNTYPE; MZUT Av5753 - skin; adult male; Ethiopia, Shewa, Adda Galla, Daimbi, 9 May 1879; collected by 0 . Antinori;

SYNTYPE; MZUT Av5754 - skin; immature male; Ethiopia, Shewa, Adda Galla, Daimbi, 10 April 1879; collected by 0. Antinori;

SYNTYPE; MZUT Av5755 - skin; female; Ethiopia, Shewa, Adda Galla, Daimbi, 9 April 1879; collected by 0 . Antinori.

Notes: Salvadori (1915: 32) does not cite specimens n. 5754 and n. 5755 as types, but reports the $\mathrm{n}$. 5757, which actually corresponds to a different species.

Dinemellia ruspolii Salvadori, 1894

Salvadori T., 1894. Mem. R. Acc. Sc. Torino, serie 2a, vol. 44: 558.

Now: Dinemellia dinemelli dinemelli (Rüppell, 1845)

HOLOTYPE; MZUT Av11142 - skin; Somalia, Banan, 1891; collected by E. Ruspoli.

Euplectes scioanus Salvadori, 1884

Salvadori T., 1884. Ann. Mus. Civ. Genova, XXI: 185-186.

Now: Euplectes afer strictus Hartlaub, 1857

SYNTYPE; MZUT Av11944 (former 5564) - skin; adult male breeding; Ethiopia, Shewa, Tuor-Amesh, 18 August 1877; collected by 0. Antinori;

SYNTYPE; MZUT Av17768 (former 5565) - skin; adult male breeding; Ethiopia, Shewa, Tuor-Amesh, 1 September 1877; collected by 0. Antinori.

Notes: the specimens are indicated as types by Elter (1986: 202) but not by Salvadori (1915). 
Urobrachia traversii Salvadori, 1888

Salvadori T., 1888. Ann. Mus. Civ. Genova, XXVI: 287-88.

Now: Euplectes axillaris traversii (Salvadori, 1888)

SYNTYPE; MZUT Av5522 - skin; male; Ethiopia, Shewa, 'Sutta' or 'Sululta', 9 September 1885; collected by V. Ragazzi.

Notes: Salvadori (1888b: 287) describes the specimen of the Ragazzi collection and reports that he also examined a $O$ from Leopoldo Traversi coll., previously misidentified as Pentheria axillaris by Giglioli (1888: 30). This specimen is not present in the MZUT collection.

Textor castaneo-auratus Antinori, 1864

Antinori 0., 1864. Catalogo descrittivo di una collezione di uccelli fatta da Orazio Antinori nell'interno dell'Affrica centrale nord dal maggio 1859 al luglio 1861. Milano, p. 65.

Now: Ploceus badius badius (Cassin, 1850)

SYNTYPE; MZUT Av5655 - dismounted skin; adult male breeding; Ethiopia, Shewa, WadiMedine, Antub, September 1859; collected by 0. Antinori (field number 284).

Notes: Salvadori $(1915: 18,47)$ and Elter (1986: 457) quotes the types specimens n. 5655 (but 5665 in Salvadori, erroneously) and n. 5657. However, specimen Av5657 is not the second type indicated by Antinori (1864: 65), but another bird that Antinori (1864: 63) misidentified as Textor galbula. Only subsequently Salvadori (1870: 740) identified this specimen, n. 293 of the Antinori collection, as Hyphantornis badius Cassin, senior synonym of Textor castaneoauratus.

\section{ESTRILDIDAE}

Lagonosticta ugandae Salvadori, 1906

Salvadori T., 1906. Boll. Mus. Zool. An. comp. Univ. Torino, No. 542 (vol. XXI): 2.

Now: Lagonosticta rubricata congica Sharpe, 1890

HOLOTYPE; MZUT Av5653 - skin; Zaire, Fort Portal, July 1906; collected by A. Roccati, during Duca degli Abruzzi travel to Ruwenzori.

Estrilda ochrogaster Salvadori, 1897

Salvadori T., 1897. Boll. Mus. Zool. An. comp. Univ. Torino, No. 287 (vol. XII): 4.

Now: Estrilda paludicola ochrogaster Salvadori, 1897

HOLOTYPE; MZUT Av4932 - skin; adult male; Ethiopia, no date; donated by padre Calvi in 1842.

Estrelda nigrimentum Salvadori, 1888

Salvadori T., 1888. Ann. Mus. Civ. Genova, XXVI: 281.

Now: Estrilda erythronotos charmosyna (Reichenow, 1881)

HOLOTYPE; MZUT Av5670 - skin; adult; Ethiopia, Shewa, Farre', 7 February 1886; collected by V. Ragazzi (field number 460).

Cryptospiza salvadorii Reichenow, 1892

Reichenow A., 1892. Journ. f. Orn., XL, Heft II: 187.

Now: Cryptospiza salvadorii salvadorii Reichenow, 1892

HOLOTYPE; MZUT Av5575 - skin; adult male; Ethiopia, Sciotalit, 23 September 1879; collected by 0 . Antinori (field number 1001).

Notes: Av5575 is the specimen misidentified by Salvadori (1884: 180) as Cryptospiza reichenowi Hartlaub, on which Reichenow based his new species $C$. salvadorii (Matschie et Reichenow, 1892: 221).

Munia caniceps Salvadori, 1876

Salvadori T., 1876. Ann. Mus. Civ. Genova, IX: 38.

Now: Lonchura caniceps caniceps (Salvadori, 1876)

SYNTYPE; MZUT Av5640 - skin; adult male; Papua New Guinea, Hall Sound, Naiabui, August 1875; collected by L.M. D’Albertis (field number 404); 
SYNTYPE; MZUT Av5641 - skin; immature male; Papua New Guinea, Hall Sound, Naiabui, August 1875; collected by L.M. D’Albertis (field number 400).

\section{PASSERIDAE}

Petronia stulta subsp. puteicola Festa, 1894

Festa E., 1894. Boll. Mus. Zool. An. comp. Univ. Torino, No. 174 (vol. IX): 3-4.

Now: Petronia petronia puteicola Festa, 1894

HOLOTYPE; MZUT Av5259 - skin; adult male; Jordan, Madaba, 25 April 1893; collected by E. Festa.

\section{FRINGILLIDAE}

Coccothraustes insularis Salvadori \& Festa, 1914

Salvadori T., Festa E., 1914. Boll. Mus. Zool. An. comp. Univ. Torino, No. 681 (vol. XXIX): $1-2$.

Now: Coccothraustes coccothraustes coccothraustes (Linnaeus, 1758)

SYNTYPE; MZUT Av5159 - skin; adult male; Italy, Sardinia, Pantaleo, 24 June 1912; collected by E. Festa (field number 57);

SYNTYPE; MZUT Av5161 - skin; adult male; Italy, Sardinia, Pantaleo, 24 June 1912; collected by E. Festa (field number 53);

SYNTYPE; MZUT Av5162 - skin; adult female; Italy, Sardinia, S. Antioco, 26 June 1912; collected by E. Festa (field number 58);

SYNTYPE; MZUT Av5163 - skin; adult female; Italy, Sardinia, Pantaleo, 24 June 1912; collected by E. Festa (field number 52);

SYNTYPE; MZUT Av5164 - skin; adult female; Italy, Sardinia, S. Antioco, 28 June 1912; collected by E. Festa (field number 63);

SYNTYPE; MZUT Av5165 - skin; immature male; Italy, Sardinia, S. Antioco, 27 June 1912; collected by E. Festa (field number 62).

Serinus reichenowi Salvadori, 1888

Salvadori T., 1888. Ann. Mus. Civ. Genova, XXVI: 272-273.

Now: Crithagra reichenowi (Salvadori, 1888)

SYNTYPE; MZUT Av5337 - skin; male; Ethiopia, Shewa, "Cialalakà" Lake (Cheleleka or Dabashi Lake), 2 June 1885; collected by V. Ragazzi (field number 310);

SYNTYPE; MZUT Av5338 - skin; female; Ethiopia, Shewa, "Cialalakà" Lake, 8 June 1885; collected by V. Ragazzi (field number 269).

Serinus flavigula Salvadori, 1888

Salvadori T., 1888. Ann. Mus. Civ. Genova, XXVI: 272.

Now: Crithagra xanthopygia (Rüppell, 1840)

SYNTYPE; MZUT Av5340 - skin; adult female; Ethiopia, Shewa, Ankober, 17 May 1885; collected by V. Ragazzi (field number 183);

SYNTYPE; MZUT Av5339 - skin; female; Ethiopia, Shewa, "Kolla di Aigaber", 16 October 1880; collected by O. Antinori (field number 1374).

Notes: Salvadori (1888b: 272) describes S. flavigula referring to the specimens n. 183 and n. 512 of the Ragazzi collection and to n. 1374 of the Antinori collection, which was previously misidentified as Crithagra xanthopygia Rüppell (Salvadori 1884: 172). An old label on the specimen Av5339 clearly reads "Typus!"

A few years later, apparently without reason, Salvadori (1915: 34) reported as types only the two specimens of the Ragazzi collection. Currently, only specimens Av5339 and Av5340 are hosted in the collection of the Turin Museum.

\section{PASSERELLIDAE}

Buarremon borellii Salvadori, 1897

Salvadori T., 1897. Boll. Mus. Zool. An. comp. Univ. Torino, No. 292 (vol. XII): 6-7. 
Now: Arremon torquatus borellii (Salvadori, 1897)

HOLOTYPE; MZUT Av3636 - skin; adult male; Argentina, Jujuy, San Lorenzo, May 1896; collected by A. Borelli (field number 408).

\section{EMBERIZIDAE}

Fringillaria poliopleura Salvadori, 1888

Salvadori T., 1888. Ann. Mus. Civ. Genova, XXVI: 269-71.

Now: Fringillaria poliopleura Salvadori, 1888

HOLOTYPE; MZUT Av5048 - skin; adult male; Ethiopia, Shewa, Sodde', 2 August 1886; collected by V. Ragazzi (field number 792).

Emberiza cerrutii De Filippi, 1863

De Filippi F., 1863. Arch. Zool. Anat. Fisiol., vol. II, fasc. II: 383-384.

Now: Emberiza buchanani cerrutii De Filippi, 1863

SYNTYPE; MZUT Av2174 - dismounted skin; female/immature plumage; Iran, 1862; collected by F. De Filippi;

SYNTYPE; MZUT Av2282 - dismounted skin; adult male; Iran, 1862; collected by F. De Filippi.

Emberiza palustris Savi, 1829

Savi P., 1829. Ornitologia Toscana. Vol. II, pagg. 91-94.

Emberiza compilator Mathew \& Iredale, 1920

Mathews G.M. \& Iredale T., 1920. The Austral Avian Record, vol. IV (4-5): 131.

Now: Schoeniclus schoeniclus intermedius (Degland, 1849)

SYNTYPE (possible); MZUT Av1375 - mounted skin; adult male, winter plumage; Italy, Piedmont, 26 February 1816; donated by Dr. Ferrero.

SYNTYPE (possible); MZUT Av1378 - mounted skin; adult male, winter plumage; Italy, Piedmont, October 1821.

SYNTYPE (possible); MZUT Av14467 (old n. 1380) - mounted skin; adult female, winter plumage; Italy, Piedmont, 2 November 1822.

Notes: these specimens were not listed among the types by Salvadori (1915) and by Elter (1986: 192). However, Savi (1829: 92) wrote: "The Bunting of which I am speaking here [...], in the Turin Museum and in the Jardin des Plantes of Paris is preserved as a mere variety of the Emberiza schoeniclus". So, probably Savi had seen the specimens MZUT Av1375, Av1378 and Av14467, which were already in Turin for several years (right location and right time). The labels with the indication Emberiza palustris represent further confirmation of their status. Emberiza compilator is the nomen novum introduced by Mathews et Iredale (1920: 131) to replace the binomen of Savi, which was preoccupied by Embriza (sic) palustris Frenzel, 1801. The type specimens of E. palustris are therefore also types of $E$. compilator.

\section{THRAUPIDAE}

Rhamphocoelus festae Salvadori, 1896

Salvadori T., 1896. Boll. Mus. Zool. An. comp. Univ. Torino, No. 249 (vol. XI): 1-2.

Now: Ramphocelus passerinii costaricensis Cherrie, 1891 according to Griscom (1932: 202); most likely a hybrid between $R$. dimidiatus and $R$. passerinii according to Olson et Violani (1995: 303).

HOLOTYPE; MZUT Av11494 - skin; male; Panama, Chiriquì; 1895; purchased by Festa (from Arcé) and donated to the Turin Museum.

Spermophila plumbeiceps Salvadori, 1895

Salvadori T., 1895. Boll. Mus. Zool. An. comp. Univ. Torino, No. 208 (vol. X): 5-6.

Now: Sporophila ruficollis Cabanis, 1851

HOLOTYPE; MZUT Av11352 - skin; immature male; Argentina, Tucuman, San Pablo, December 1893; collected by A. Borelli (field number 320). 
Spermophila aequatorialis Salvadori \& Festa, 1899

Salvadori T., Festa E., 1899. Boll. Mus. Zool. An. comp. Univ. Torino, No. 357 (vol. XIV): 24-25.

Now: Sporophila corvina ophthalmica (P. L., Sclater, 1860)

SYNTYPE; MZUT Av5390 - skin; adult male; Ecuador, Balzar, December 1897; collected by E. Festa (field number 2739);

SYNTYPE; MZUT Av5391 - skin; female; Ecuador, Balzar, December 1897; collected by E. Festa (field number 2772).

Notes: Salvadori et Festa (1899a: 24) match the specimen Av5390 with the No. 2735 of the Festa collection, however the original label reads No. 2739.

Certhiola intermedia Salvadori \& Festa, 1899

Salvadori T., Festa E., 1899. Boll. Mus. Zool. An. comp. Univ. Torino, No. 357 (vol. XIV): 13-14.

Now: Coereba flaveola intermedia (Salvadori \& Festa, 1899).

SYNTYPE; MZUT Av3759 - skin; male; Ecuador, Gualaquiza, November 1895; collected by E. Festa (field number 68);

SYNTYPE; MZUT Av3760 - skin; female; Ecuador, Gualaquiza, June 1895; collected by E. Festa (field number 489);

SYNTYPE; MZUT Av3761 - skin; male (female?); Ecuador, Zamora Valley, December 1895; collected by E. Festa (field number 215);

SYNTYPE; MZUT Av3762 - skin; male; Ecuador, Zamora Valley, December 1895; collected by E. Festa (field number 190);

SYNTYPE; no MZUT number, A-672 (Elter catalogue) - skin, immature male; Gualaquiza, no date; collected by E. Festa

Notes: specimens from Av3759 to Av3762 are quoted with wrong numbers from 3159 to 3162 in Salvadori (1915: 38). Specimen A-672 was collected by Festa in the same locality as specimen Av3760, which however should correspond to A-627 (Elter 1986: 124). The similarity of the numbers suggests a transcription error, because in the MZUT collection another specimen is registered under the number A-672 (Elter 1986: 328), but it corresponds to Av1496 Myiotheretes fumigatus (Boissonneau, 1840), also collected by E. Festa but in a different locality (Festa 1909: 291).

In any case, 5 specimens (males and females) from Gualaquiza have been described in the paper of Salvadori and Festa (1899: 24-25).

\section{STENOSTIRIDAE}

Elminia Teresita Antinori, 1864

Antinori O., 1864. Catalogo descrittivo di una collezione di uccelli fatta da Orazio Antinori nell'interno dell'Affrica centrale nord dal maggio 1859 al luglio 1861. Milano, pp. 50-51.

Now: Elminia longicauda teresita Antinori, 1864

SYNTYPE; MZUT Av5543 - male; Etiopia, Djur, 14 March 1861; collected by O. Antinori (field number 125);

SYNTYPE; MZUT Av5544 - female; Etiopia, Djur, 14 March 1861; collected by O. Antinori (field number 135).

Notes: specimens Av5543 e Av5544 quoted with numbers 5343 and 5344 in Salvadori (1915: 18).

\section{PARIDAE}

Melaniparus semilarvatus Salvadori, 1866

Salvadori T., 1866. Atti Soc. It. Sc. Nat., vol. VIII [1865], fasc. 4: 377-378.

Now: Sittiparus semilarvatus semilarvatus (Salvadori, 1865)

SYNTYPE; MZUT Av1273 - dismounted skin; immature / female; Philippines, Luzon, no 
date; from Callery collection in 1842;

SYNTYPE; MZUT Av1285 - dismounted skin; adult male; Philippines, no date; from Callery collection in 1842 .

Note: the catalogue numbers are those indicated by Salvadori (1866a), however the collector and the provenance indicated in the original description and on an old label ("Melaniparus semilarvatus, Salvad. / Imalaya (Solaroli) / Tipo!") were completely wrong. Salvadori (1879a: 300-303, pl. IX) published a figure of the species, along with some doubts about its origin, but the true locality of the two syntypes of Melaniparus semilarvatus was identified only a few years later (Gadow 1883: 38 -39, Sharpe 1903: 333, Salvadori 1915: 12).

Dickinson et Christidis (2014: 428), following Snow (in Paynter 1967: 119), indicate 1865 as the date of description, however the date should be corrected to 1866 , because the issue n. 4 , vol. VIII, of the Atti della Società Italiana di Scienze Naturali is dated February 1866 (Galbreath et Aimassi 2020: 147).

Parus pallidiventris Reichenow, 1885

Reichenow A., 1885. Journ. f. Orn., XXXIII, N. 170 (April): 217-218.

Now: Melaniparus rufiventris pallidiventris (Reichenow, 1885).

SYNTYPE; MZUT Av11112 - dismounted skin; adult; Kakoma, Tabora Region, Tanzania, 24 August 1881; collected by R. Böhm; donated by A. Reichenow from the collections of the ZBM, Berlin.

Notes: Salvadori (1915) does not list this bird among type specimens.

\section{REMIZIDAE}

Anthoscopus Roccatii Salvadori, 1906

Salvadori T., 1906. Boll. Mus. Zool. An. comp. Univ. Torino, No. 542 (vol. XXI): 2.

Now: Anthoscopus caroli roccatii Salvadori, 1906

HOLOTYPE; MZUT Av3919 - skin; immature; Uganda, Entebbe, 1906; collected by A. Roccati during the Duca degli Abruzzi expedition to Ruwenzori.

\section{ALAUDIDAE}

Certhilauda doriae Salvadori, 1868

Salvadori T., 1868. Atti R. Acc. Sc. Torino, vol. III: 292.

Now: Alaemon alaudipes doriae (Salvadori, 1868)

HOLOTYPE/SYNTYPE?; MZUT Av5405 - dismounted skin; Iran, Bender-Abbas, 1 December 1862; collected by G. Doria.

Notes: the label attached to the pedestal of the specimen Av5405 cites “Typus!". Salvadori (1868b: 291) wrote "the specimen of Bender Abbas" and Salvadori (1915: 17) cited the specimen n. 5405 of the Turin Museum as "the type", suggesting that it is unique, however Arbocco et al. (1979: 208) report the presence of the holotype in the Genoa Museum (C.E. 4500) with the same capture data as MZUT Av5405.

Megalophonus rufo-cinnamomeus Salvadori, 1866

Salvadori T., 1866. Atti Soc. It. Sc. Nat., vol. VIII [1865], fasc. 4: 378-379.

Now: Mirafra rufocinnamomea rufocinnamomea (Salvadori, 1865)

HOLOTYPE; MZUT Av3604 - dismounted skin; Ethiopia, Abyssinia, no date; donated by Calvi in 1842.

Notes: Dickinson et Christidis (2014: 440), following Peters (in Mayr et Greenway 1960: 13), indicate 1865 as the date of description, however the date should be corrected to 1866, because the issue n. 4, vol. VIII, of the Atti della Società Italiana di Scienze Naturali is dated February 1866 (Galbreath et Aimassi 2020: 147).

Mirafra erythrocephala Salvadori \& Giglioli, 1885

Salvadori T., Giglioli E. H., 1885. Atti R. Acc. Sc. Torino, vol. XX: 345. 
Now: Mirafra erythrocephala Salvadori \& Giglioli, 1885

SYNTYPE; MZUT Av20290 (former 8562) - dismounted skin; Vietnam, Thu-dau-mot, HoChi-Minh, June 1866; collected by E.H. Giglioli \& F. De Filippi (Magenta voyage).

Notes: specimen Av6090, with the same capture data of Av20290, was no longer found in the Turin collection. Violani et Barbagli (1999) established that Mirafra erythrocephala is a valid name and that Mirafra assamica marionae Baker, 1915 is a junior synonym.

Otocoris larvata De Filippi, 1863

De Filippi F., 1863. Arch. Zool. Anat. Fisiol., vol. II, fasc. II: 382.

Now: Eremophila alpestris albigula (Bonaparte, 1850)

SYNTYPE; MZUT Av5406 - dismounted skin; adult male; Iran, Mazandaran, Damavand, 1862; collected by F. De Filippi;

SYNTYPE; MZUT Av5407 - skin; female; Iran, Mazandaran, Damavand, 1862; collected by F. De Filippi;

SYNTYPE; MZUT Av5518 - skin; immature (male?); Iran, Mazandaran, Damavand, 1862; collected by F. De Filippi.

Notes: De Filippi (1863: 382) claims that the species "is similar to the O. penicillata Gould" and that "it is abundant in the mountains surrounding the Demavend", but does not indicate any specimen. Salvadori (1868b: 285) does not comment on the validity of this taxon because he believes no type specimen has been collected. Subsequently, Salvadori himself (1915: 17) refers that the Turin Museum houses three specimens, collected in Persia by De Filippi (field numbers 5406, 5407, 5518) which are perhaps the types of Otocoris larvata that, according to Hartert (1910: 262), is a junior synonym of Otocoris albigula. Elter (1986: 337) considers the three specimens of Turin Museum as syntypes.

\section{MACROSPHENIDAE}

Sphenoeacus natalensis Shelley, 1882

Shelley G.E., 1882. Proc. Zool. Soc. London for 1882: 336-337.

Now: Sphenoeacus afer natalensis Shelley, 1882

SYNTYPE; MZUT Av11079 - male; South Africa, KwaZulu-Natal, Newcastle, 25 June 1881; collected by A.C. Butler; from Captain Shelley in 1887;

SYNTYPE; MZUT Av11080 - female; South Africa, North West (Transvaal), Rustenburg, 8 July 1878; collected by W. Lucas; from Captain Shelley in 1887.

Notes: Salvadori (1915: 33) points out that in 1887 Captain E. Shelley sent to the Turin Museum a "truly precious collection of 114 African specimens" but does not mention type-specimens. Elter (1986: 434), who almost always follows the indications of Salvadori, cites Shelley's specimens, but does not consider them as types.

In fact, we believe that the specimens Av11079 and Av11080 are both syntypes, because they were in the availability of Shelley before the description of the species and were collected by people and in places that the same Shelley (1882: 337) indicates in describing his new species. In the Tring Museum (Warren et Harrison 1971: 372) there is a syntype with the same capture data as Av11079, but the date is not indicated.

\section{CISTICOLIDAE}

Eremomela (?) canescens Antinori, 1864

Antinori 0., 1864. Catalogo descrittivo di una collezione di uccelli fatta da Orazio Antinori nell'interno dell'Affrica centrale nord dal maggio 1859 al luglio 1861. Milano, pp. 38-39. Now: Eremomela pusilla canescens Antinori, 1864

SYNTYPE; MZUT Av5587 - dismounted skin; adult male; Ethiopia, Djur, July 1864; collected by 0 . Antinori (field number 50 );

SYNTYPE; MZUT Av5588 - dismounted skin; adult; Ethiopia, Djur, July 1864; collected by O. Antinori (field number 98). 
Drymoeca antinorii Heuglin, 1867

Heuglin M. T. von, 1867. Journal für Ornithologie, XV: 202.

Now: Cisticola lateralis antinorii (von Heuglin, 1869)

HOLOTYPE; MZUT Av5632 - skin; Ethiopia, Djur, 11 April 1861; collected by O. Antinori (field number 188).

Notes: Mayr et Cottrell (1986: 88) and Dickinson et Christidis (2014: 456) claim that the species was first described by Heuglin (1869: 102), however we believe that an appropriate description had already been given by Heuglin (1867: 202), as indicated in Zoonomen (Zoological Nomenclature Resource, version 05-Jan-12, accessed 26 April 2017). Heuglin's description (1867: 202) is correct because it makes explicit reference to the specimen cited by Antinori (1864: 37).

Drymoica (?) troglodytes Antinori, 1864

Antinori 0., 1864 - Catalogo descrittivo di una collezione di uccelli fatta da Orazio Antinori nell'interno dell'Affrica centrale nord dal maggio 1859 al luglio 1861. Milano, p. 38.

Now: Cisticola troglodytes troglodytes (Antinori, 1864)

SYNTYPE; MZUT Av5589 - dismounted skin; adult male; Ethiopia, Djur, July 1864; collected by 0 . Antinori (field number 49);

SYNTYPE; MZUT Av5590 - dismounted skin; adult female; Ethiopia, Djur, July 1864; collected by 0. Antinori (field number 108).

Prinia sonitans Swinhoe, 1860

Swinhoe R., 1860. Ibis, 2 (N. V, January): 50.

Now: Prinia flaviventris sonitans Swinhoe, 1860

SYNTYPE (possible); MZUT Av5564 - dismounted skin; adult; China, Amoy, ca. 1860; from R. Swinhoe collection; donated by Giglioli in 1865.

Notes: qualified as "type" in MS. Catalogue but not listed among type specimens in Salvadori (1915) and in Elter (1986: 386).

Drymoica extensicauda Swinhoe, 1860

Swinhoe R., 1860. Ibis, 2 (N. 5, January): 50-51.

Now: Prinia inornata extensicauda (Swinhoe, 1860)

PARATYPE (possible); MZUT Av5565 - China, Amoy, ca. 1860; from R. Swinhoe collection; donated by Giglioli in 1865.

Notes: qualified as "type" in MS. Catalogue but not listed among type specimens in Salvadori (1915) and in Elter (1986: 386). The holotype is hosted in the NHMUK of Tring, according to Warren et Harrison (1971: 171); Sanchez Osés (2010: 50) reports a paratype in the Übersee-Museum Bremen.

Drymoca hypoxantha Sharpe, 1877

Sharpe R.B., 1877. The Birds of South Africa by Edgar Leopold Layard, new ed., Part. 4, p. 260-261.

Now: Prinia hypoxantha (Sharpe, 1877)

PARALECTOTYPE; MZUT Av11061 - skin; adult female; South Africa, KwaZulu, Pinetown; 19 January 1875; collected by Ayres (or Layard?); from Shelley.

Notes: the specimen Av11061 is not listed as a type in Salvadori (1915) and in Elter (1986: 386). A lectotype and two paralectotypes are housed in Tring's NHMUK (Warren et Harrison 1971: 248). Because in describing this taxon Sharpe (1877: 260) also refers to the specimens of Captain Shelley "from the neighborhood of Pinetown", the specimen Av11061 can be considered a paralectotype.

Orthotomus borneoensis Salvadori, 1874

Salvadori T., 1874. Ann. Mus. Civ. Genova, V: 247-248.

Now: Orthotomus ruficeps borneoensis Salvadori, 1874

SYNTYPE; MZUT Av6826 - dismounted skin; adult male; Malaysia, Borneo, Sarawak, June 1865; collected by G. Doria. 


\section{LOCUSTELLIDAE}

Sylvia Luscinioides Savi, 1824

Savi P., 1824. Nuovo Giornale de' letterati, Tomo settimo, N. 14: 341-343.

Now: Locustella luscinioides luscinioides (Savi, 1824)

SYNTYPE; MZUT Av1006 - mounted skin; adult male; Italy, Tuscany, near Pisa, 10 April 1823; collected by P. Savi;

SYNTYPE; MZUT Av1008 - dismounted skin; adult; Italy, Tuscany, 1823; collected by P. Savi.

Notes: in describing the species Savi (1824: 341) explicitly cites a specimen captured before or during the autumn of 1821, however in the paragraph "Observation" (p. 342) indicates the variability present in individuals of different sexes, captured in different seasons. We can therefore deduce that Savi examined numerous individuals, including Av1006 and Av1008. Salvadori (1915: 6) indicates as "cotype" only the specimen n. 1006 and reports a second specimen from P. Savi (n. 1007), captured in 1824. Elter (1986: 446) indicates as types the specimens n. 1006 and n. 1007 and lists separately the n. 1008 (p. 277). We have no longer found the specimen n. 1007 in the MZUT collection.

\section{HIRUNDINIDAE}

Psalidoprocne pallidigula Salvadori, 1907

Salvadori T., 1907. Boll. Mus. Zool. An. comp. Univ. Torino, No. 570 (vol. XXII): 4.

Now: Psalidoprocne albiceps albiceps P.L. Sclater, 1864

HOLOTYPE; MZUT Av3094 - skin; adult; Zaire, Moero Lake, Lukonzolwa, 1892; from Ascenso collection.

Psalidoprocne antinorii Salvadori, 1884

Salvadori T., 1884, Ann. Mus. Civ. Genova, Serie 2a, Vol. 1 (XXI): 123.

Now: Psalidoprocne pristoptera antinorii Salvadori, 1884

HOLOTYPE; MZUT Av2869 - skin; male; Ethiopia, Shewa, Dens, 27 May 1880; collected by 0. Antinori (field number 1298).

Notes: Salvadori (1915: 32) and Elter (1986: 389) erroneously consider as a type also the specimen Av2871 (collected 5 June 1885 by Ragazzi).

Collocalia ariel Gould, 1843

Gould J., 1843. Proc. Zool. Soc. London, Part X [1842]: 132.

Now: Petrochelidon ariel (Gould, 1842)

SYNTYPE (possible); MZUT Av3633 - dismounted skin; female; W Australia coast; donated by J. Gould in 1843.

Notes: specimen not mentioned in Salvadori (1915) but quoted ad "type" in Elter (1986: 151).

\section{PYCNONOTIDAE}

Hemixus sumatranus Salvadori, 1888

Salvadori T., 1888, Ann. Mus. Civ. Genova, Serie 2a, Vol. 5 (XXV): 525-527.

Now: Ixos virescens sumatranus (Wardlaw Ramsay, 1882)

SYNTYPE; MZUT Av9563 - dismounted skin; male; Indonesia, Sumatra, Mount Singgalang, 9 August 1878; collected by O. Beccari (field number 106); from G. Doria.

Notes: Hemixus sumatranus Wardlaw Ramsay and Hemixus sumatranus Salvadori are both available names and are simultaneously primary homonyms and objective synonyms, as they share at least part of their type series (Aimassi et Dickinson 2018). These authors have verified that among the other seven syntypes of $H$. sumatranus Salvadori, three are hosted in the Museo Civico di Storia Naturale "Giacomo Doria", Genova (Arbocco et al. 1979), one in the Tring museum, referred to as the holotype of H. sumatranus Wardlaw 
Ramsay (Warren et Harrison 1971: 544), one in the Senckenberg Naturmuseum, Frankfurt-am-Main, referred to (erroneously) as a "typus" of H. sumatranus Wardlaw Ramsay (SysTax database; G. Mayr, pers. comm.).

Ixos virescens Temminck, 1825

Temminck in Temminck C.-J. \& Laugier de Chartrouse M., 1825. Planches Coloriées, livr. 64, pl. 382, fig. 1.

Now: Ixos virescens virescens Temminck, 1825

SYNTYPE (possible); MZUT Av539 - dismounted skin; Indonesia, Java, no date; collected by Kuhl \& Hasselt in 1820-21; from Temminck in 1826.

Notes: it is quite reasonable to suppose that this specimen is a syntype, since it was given by Temminck himself and because in the text of Planche 382 there is a fairly explicit reference to H. Kuhl. Salvadori (1888c: 526) quotes for the first time the specimen Av539 as a type of " $H$. virescens [...] collected by Kuhl and van Hasselt and sent by Temminck". Subsequently, the specimen is again referred to as type by Salvadori (1915: 7) and Elter (1986: 474) which, surprisingly, use the French name "Turdoïde verdin" as if it were a Latin name.

Brachypus urostictus Salvadori, 1870

Salvadori T., 1870. Atti R. Acc. Sc. Torino, vol. V: 509-510.

Now: Poliolophus urostictus urostictus (Salvadori, 1870)

HOLOTYPE; MZUT Av6982 - dismounted skin; male; Philippines, April 1867; purchased from Verreaux Frères.

\section{PHYLLOSCOPIDAE}

Phylloscopus sylvicultrix Swinhoe, 1860

Swinhoe R., 1860. Ibis, 2 (n. V, January): 53.

Now: Seicercus borealis borealis (J. H. Blasius, 1858)

SYNTYPE (possible); MZUT Av8260 - skin; adult male; China, Amoy, 27 April 1859; from R. Swinhoe collection; donated by Giglioli.

Notes: it is possible that Swinhoe examined this specimen while describing the species, but there is no mention of his type status in the MS. Catalogue of the Turin Museum, nor on the labels. Not treated as type in Salvadori (1915) and in Elter (1986: 364).

\section{SCOTOCERCIDAE}

Scotocerca inquieta harterti Festa, 1925

Festa E., 1925. Boll. Mus. Zool. An. comp. Univ. Torino, n.s., No. 24 (vol. XXXIX): 13.

Now: Scotocerca inquieta saharae (Loche, 1858)

HOLOTYPE; MZUT Av8344 - skin; adult male; Libya, Derna, "Zavia Mechili”, 10 March 1922; collected by E. Festa (field number 197).

Notes: not regarded as a type by Elter (1986: 425). Scotocerca inquieta harterti was still a valid name in Mayr et Cottrell (1986: 126).

Sylvia Cetti Marmora, 1820

Marmora A. della, 1820. Mem. R. Acc. Sc. Torino, vol. 25: 253-259.

Now: Cettia cetti (Temminck, 1820)

SYNTYPE; MZUT Av996 - mounted skin; adult male; France, Nice, Var river, 8 November 1819; collected by A. della Marmora.

Notes: Salvadori (1915: 5) claims that the specimen Av996 is "undoubtedly one of the types of the species", while according to Elter (1986: 445) also Av995 (adult male, Sardinia, 9 December 1820) and Av997 (adult female, Sardinia, 16 November 1820) would be type specimens.

Alberto della Marmora (1820: 257) described S. cetti having at his disposal five birds, two collected in Sardinia (Iglesias, near Cagliari) and three in Nice (France). The specimens of the Museum of Turin that come from Sardinia (those indicated by Elter) cannot be part 
of the type series, because they were captured in the months of November and December 1820, while the "Memoria" of Marmora had already been read at the Academy of the Sciences of Turin on July 9 th.

At least one of the specimens captured in Sardinia by Marmora before the description of Sylvia cetti (hence a syntype) was sent by Bonelli to Temminck, who used it to describe "his" Sylvia cetti, even if he attributed the species to Alberto della Marmora (Marmora 1820: 257, Bonelli 1820: 261, Temminck 1820: 194). Thus, these specimens are syntypes also of $S$. cetti Temminck and the two binomina are simultaneously primary homonyms and objective synonyms. Unfortunately, the syntype of the Leiden Museum, coming from Sardinia, does not seem to have been attributed to Marmora (Dekker 2003: 53).

Witherby (1920: 322, in footnote) stated that Temminck's "Manuel" was published in October (the date is clearly specified in the title page of the book), while the Memoria of Marmora was published in November or December.

Homochlamys luscinia Salvadori, 1870

Salvadori T., 1870. Atti R. Acc. Sc. Torino, vol. V: 511-512.

Now: Horornis canturians canturians (Swinhoe, 1860)

HOLOTYPE; MZUT Av4973 - dismounted skin; female; Philippines, 1843; from Callery collection.

Notes: cited by mistake with the n. 4473 in Salvadori (1915: 22).

\section{AEGITHALIDAE}

Acredula sicula Whitaker, 1901

Whitaker J.I.S., 1901. Bulletin of B.O.C. 11: 51-52.

Now: Aegithalos caudatus siculus (Whitaker, 1901)

SYNTYPE; MZUT Av12767 - dismounted skin; adult male; Italy, Sicily, Palermo, Bosco della Ficuzza, 8 February 1901; from Whitaker collection in 1905.

Notes: the specimens Av12767 and Av12768 are quoted as "cotypes, or topotypes" in Salvadori (1915: 40) and as syntypes in Elter (1986: 56).

In describing Acredula sicula Whitaker (1901: 51-52) "exibited an exemple" (i.e. one specimen) of a new species and said that in the Museum of Palermo he "had seen a solitary example of this Acredula, obtained some years ago [March 1888] in the same wood [bosco di Ficuzza, Palermo] from which his specimens [i.e. more than one] had been procured".

Whitaker (1902: 54) talks about "specimens of the bird having been exhibited the meeting of that Club [the B.O.C.] held on the 20th of March, 1901". So, it is not clear if just one or more specimens were presented to the meeting. In the same paper, on $\mathrm{p}$. 58, Whitaker provides a "full description of the types of this Acredula in my collection": a male and a female both "from Bosco di Ficuzza, Sicily; February 3rd, 1901".

The wording, literally, only allows to deduce that the two specimens mentioned, housed in the Whitaker collection, are to be considered as syntypes. Certainly, these are the specimens quoted by Warren et Harrison (1971: 508).

We believe that, according to Art. 72.4.1 and 72.2.1.1 (ICZN, 1999), the specimen of the Museum of Palermo and all the specimens obtained from Whitaker from the "Bosco di Ficuzza" before 20 March 1901, should be included in the type series.

The specimen Av12767, collected on 8 February 1901, should therefore be considered as syntype. The specimen Av12768, collected on the same locality but on 9 October 1901, evidently is not part of the type series.

\section{SYLVIIDAE}

Curruca cinerea var. persica De Filippi, 1865

De Filippi F., 1865. Note di un viaggio in Persia nel 1862. Milano, pag. 348.

Now: Curruca communis communis (Latham, 1787) 
SYNTYPE; MZUT Av2370 - dismounted skin; immature or female; Iran, 1862; collected by F. De Filippi;

SYNTYPE; MZUT Av2402 - dismounted skin; Iran, 1862; collected by F. De Filippi.

Notes: the description by De Filippi (1865) is found in the text on p. 162; in the same work but on p. 348, the subspecies is quoted in a list and the locality "from Delidian onwards, very common" is indicated.

Suthora feae Salvadori, 1889

Salvadori T., 1889a. Ann. Mus. Civ. Genova, ser. 2a, vol. 7 (XXVII): 363.

Now: Suthora nipalensis feae Salvadori, 1889

SYNTYPE; MZUT Av10968 - dismounted skin; male; Myanmar, Tounghoo, Keren Hills, 5 March 1888; collected by L. Fea (field number 910).

\section{ZOSTEROPIDAE}

\section{Z. [Zosterops] simplex Swinhoe, 1861}

Swinhoe R., 1861. Ibis, III (No. XII): 331.

Now: Zosterops japonicus simplex Swinhoe, 1861

PARALECTOTYPE; MZUT Av5570 - dismounted skin; China, Amoy, April 1861; donated by R. Swinhoe to E. Giglioli.

Notes: specimen Av5570 quoted as type in Elter (1986: 489) but not in Salvadori (1915); one paralectotype in the Tring Museum (Warren et Harrison 1971: 312); one lectotype and two paralectotypes at Leiden (Dekker et Quaisser 2006: 23).

Zosterops mesoxantha Salvadori, 1889

Salvadori T., 1889b. Ann. Mus. Civ. Genova, ser. 2a, vol. 7 (XXVII): 396.

Now: Zosterops palpebrosus siamensis Blyh, 1867

SYNTYPE; MZUT Av10951 - dismounted skin; male; Myanmar, Keren, Taho', 11 March 1888; collected by L. Fea (field number 856);

SYNTYPE; MZUT Av10952 - dismounted skin; female; Myanmar, Keren, Taho', 11 March 1888; collected by L. Fea (field number 855).

Zosterops incerta Salvadori, 1892

Salvadori T., 1892. Ann. Mus. Civ. Genova, Serie 2a, Vol. 12 (XXXII): 133-134.

Zosterops salvadorii Meyer \& Wiglesworth, 1894

Meyer A. B., Wiglesworth L. W., 1894. Journ. f. Orn., XLII (No. 2): 115.

Now: Zosterops salvadorii Meyer \& Wiglesworth, 1894

SYNTYPE (of both species); MZUT Av11448 - dismounted skin; adult male; Indonesia, Enggano Is., Bua Bua, 15 May 1891; collected by E. Modigliani (field number 70).

Notes: the specimen is a syntype of both species; $Z$. salvadorii is a nomen novum, introduced because the name used by Salvadori was pre-occupied.

Zosterops novae guineae Salvadori, 1878

Salvadori T., 1878. Ann. Mus. Civ. Genova, XII: 341.

Now: Zosterops novaeguineae novaeguineae Salvadori, 1878

SYNTYPE; MZUT Av4174 - skin; male; Indonesia, New Guinea, Arfak Mts., June 1874; collected by A.A. Bruijn.

\section{LEIOTHRICHIDAE}

Alcippe morrisonia Swinhoe, 1863

Swinhoe R., 1863. Ibis, V, No. XIX: 296-297.

Now: Alcippe morrisonia morrisonia Swinhoe, 1863

SYNTYPE; MZUT Av17770 (former 5567) - dismounted skin; Taiwan, April 1862; donated by R. Swinhoe to E. Giglioli.

Notes: quoted as a type by Elter (1986: 67) but not by Salvadori (1915). 
Ixos leucopygius Rüppell, 1837

Rüppell E., 1837 [1840]. Neue Wirbelthiere zu der Fauna von Abyssinien gehörig. Vögel, pag. 82-84 [1840], taf. 30, fig. 1 [1837].

Now: Turdoides leucopygia leucopygia (Rüppell, 1837)

PARALECTOTYPE; MZUT Av536 - dismounted skin; immature? Ethiopia, Abyssinia, no date; collected by E. Rüppell.

Notes: Rüppell collected bird specimens in Africa in 1822-1827 and in 1831-1834 (Steinheimer 2005b: 165). "He gave all bird specimens MS. names, so most of the new names were already on the skins when they arrived at Frankfurt. We know from Leiden and London museums (now Tring) that these specimens were forwarded with the MS. names attached" (F. D. Steinheimer, pers. comm. to G. Aimassi, 1 October 2015). Different dates of publication for text and plate are according to Steinheimer (2005b: 211, footnote 105).

Dryonastes propinquus Salvadori, 1913

Salvadori T., 1913. Ann. Mus. Civ. Genova, ser. 3a, vol. 6 (XLVI): 5.

Now: Garrulax chinensis propinquus (Salvadori, 1913)

PARATYPE; MZUT Av11010 - dismounted skin; adult male; Myanmar, Tenasserim, Thagata, 17 April 1887; collected by L. Fea.

Notes: Salvadori (1913: 5) introduced the name Dryonastes propinquus for the specimens that he had previously identified as Dryonastes chinensis (Salvadori 1888d: 603) and designated as type the specimen "a" (field number 349), housed in the Museum of Genoa. The holotype is still hosted at the Museo civico di Storia Naturale di Genova, along with a paratype (Arbocco et al. 1979: 217).

Leiothrix laurinae Salvadori, 1879

Salvadori T., 1879. Ann. Mus. Civ. Genova, vol. XIV: 231-232.

Now: Leiothrix argentauris laurinae Salvadori, 1879

SYNTYPE; MZUT Av9570 - dismounted skin; adult male; Indonesia, Sumatra, 1877; collected by 0. Beccari (field number 7); from G. Doria;

SYNTYPE; MZUT Av9571 - mounted skin; Indonesia, Sumatra, 1877; collected by 0. Beccari; from G. Doria.

\section{HYPOCOLIIDAE}

Hypocolius ampelinus Bonaparte, 1850

Bonaparte C. L., 1850. Conspectus Generum Avium, vol. 1, Lugduni Batavorum, pag. 336. Now: Hypocolius ampelinus Bonaparte, 1850

SYNTYPE; MZUT Av1752 - dismounted skin; adult female; Ethiopia, Abyssinia, July 1839; collected by Botta; from Leiden Museum.

Notes: Heuglin (1868: 181) specifies that C.L. Bonaparte described the species on Botta's specimens and that the type locality is Abyssinia, not California as erroneously quoted by Bonaparte (1850: 336). According to Heuglin, the types in the Museum of Leiden were two, but Dekker (2003: 38) reports the presence of three syntypes in Leiden, all from Abyssinia and collected by Botta.

\section{SITTIDAE}

Sitta Neumayer Michahelles, 1830

Michahelles M., 1830. Isis von Oken, 23: col. 814-815.

Now: Sitta neumayer neumayer Michahelles, 1830

SYNTYPE (possible); MZUT Av2070 - dismounted skin; Dalmazia, no date; from M. Michahelles in 1832.

Notes: Elter (1986: 430) indicates as syntype also the specimen Av2071, which however was donated by "a certain Mr. Heckel" of Vienna (Salvadori 1915: 9), probably J.J. Heckel 
(1790-1857), curator of zoology at the Museum of Vienna, which cannot be related to the description of Michahelles.

\section{TROGLODYTIDAE}

Thryophilus leucopogon Salvadori \& Festa, 1899

Salvadori T., Festa E., 1899. Boll. Mus. Zool. An. comp. Univ. Torino, No. 357 (vol. XIV): 6.

Now: Cantorchilus leucopogon leucopogon (Salvadori \& Festa, 1899)

SYNTYPE; MZUT Av4284 - skin; male; Ecuador, Rio Peripa, November 1897; collected by E. Festa (field number 2579);

SYNTYPE; MZUT Av4285 - skin; female; Ecuador, Rio Peripa, November 1897; collected by E. Festa (field number 2615).

\section{STURNIDAE}

Gracula enganensis Salvadori, 1892

Salvadori T., 1892. Ann. Mus. Civ. Genova, XXXII: 137-138.

Now: Gracula religiosa enganensis Salvadori, 1892

SYNTYPE; MZUT Av5930 - skin; female; Indonesia, Enggano Is., "19.01.1891" (actually 19 May 1891); collected by E. Modigliani (field number 91).

Notes: Salvadori (1915: 36) and Elter (1986: 226) erroneously consider the specimen Av5930 a "type" of Graucalus enganensis Salvadori. Also, the date (January 1872) reported in the MS. catalogue is completely wrong: all the specimens of Modigliani were collected in Enggano in the months of May and June 1891 (Salvadori 1892b: 123). The letter "c" shown on the label of the Gracula enganensis Av5930, corresponds to the bird n. 91 of the Modigliani collection, captured in Bua Bua on 19 May 1891 (Salvadori 1892b: 138).

Three syntypes of Gracula enganensis are present at the Museum of Genoa (Arbocco et al. 1979: 244); a syntype is housed in the Leiden Museum (Dekker et Quaisser 2006: 50).

Calornis sanghirensis Salvadori, 1876

Salvadori T., 1876. Ann. Mus. Civ. Genova, IX: 60.

Now: Aplonis panayensis sanghirensis (Salvadori, 1876)

SYNTYPE; MZUT Av5951 - skin; male; Indonesia, Sanghir Is., Peta', September 1875; collected by A.A. Bruijn; from the Genoa Museum.

Notes: Salvadori (1915: 31) and Elter (1986: 112) also consider Av5952 as a type, but this specimen was captured by A.A. Bruijn in Buru Is., Kajeli, a different locality from the typical one.

In the Genoa Museum there are two syntypes (Arbocco et al. 1979: 243).

\section{MUSCICAPIDAE}

Cittocincla melanura Salvadori, 1887

Salvadori T., 1887. Ann. Mus. Civ. Genova, vol. XXIV: 549-550.

Now: Kittacincla malabarica melanura Salvadori, 1887

SYNTYPE; MZUT Av10617 - skin; adult male; Indonesia, Nias Is., Lelemboli, 25 July 1886; collected by E. Modigliani (field number 100).

Notes: in the Museum of Genoa there are 6 syntypes (Arbocco et al. 1979: 212).

Niltava sumatrana Salvadori, 1879

Salvadori T., 1879. Ann. Mus. Civ. Genova, vol. XIV: 201-202.

Now: Niltava sumatrana Salvadori, 1879

SYNTYPE; MZUT Av9650; dismounted skin; male; Indonesia, Sumatra, Singgalang Mt. 1878; collected by O. Beccari; from G. Doria.

Notes: "Cotypus" on the label, however Salvadori (1915) do not list this specimen among the types. Both Elter (1986: 322) and a non-original label write as catalogue number 
9560, but this is an error (CP).

Four additional syntypes are housed in the Genoa Museum (Arbocco et al. 1979: 224).

Niltava decipiens Salvadori, 1891

Salvadori T., 1891. Ann. Mus. Civ. Genova, vol. XXXII [1892]: 49-50.

Now: Niltava grandis decipiens Salvadori, 1891

SYNTYPE; MZUT Av11457 - dismounted skin; male; Indonesia, Sumatra, Si-Rambé, 8

December 1890; collected by E. Modigliani (field number 330);

SYNTYPE; MZUT Av11458 - dismounted skin; female; Indonesia, Sumatra, Si-Rambé, 7 December 1890; collected by E. Modigliani (field number 320).

Notes: the specimens Av11457 and Av11458 correspond respectively to the specimens "g" and "p" cited by Salvadori (1891: 49). In the Museum of Genoa there are four syntypes (Arbocco et al. 1979: 223). The date of printing reported on the title page of the vol XXXII of the Annali del Museo Civico di Storia Naturale di Genova is 1892, but according to Poggi (2010: 345), the paper of Salvadori (pages 40-78) should be dated 23 December 1891.

Stoparola ruficrissa Salvadori, 1879

Salvadori T., 1879. Ann. Mus. Civ. Genova, vol. XIV: 202.

Now: Eumyias indigo ruficrissa (Salvadori, 1879)

SYNTYPE; MZUT Av9558 - male; Indonesia, Sumatra, Mt. Singgalang, 3 July 1878; collected by 0 . Beccari (field number 88); from G. Doria.

Notes: the specimen Av9559, another syntype with capture data similar to those of Av9558, has not been found. In the Museum of Genoa there are four other syntypes (Arbocco et al. 1979: 223).

Erithacus xanthothorax Salvadori \& Festa, 1913

Salvadori T., Festa E., 1913. Boll. Mus. Zool. An. comp. Univ. Torino, No. 673 (vol. XXVIII): 15.

Now: Erithacus rubecula hyrcanus Blanford, 1874

SYNTYPE; MZUT Av7710 - skin; male; Greece, Rhodes, Koskino, February 1913; collected by E. Festa (field number 20);

SYNTYPE; MZUT Av7711 - skin; female; Greece, Rhodes, Koskino, 9 February 1913; collected by E. Festa (field number 5);

SYNTYPE; MZUT Av7712 - skin; female; Greece, Rhodes, Kattabia, 21 March 1913; collected by E. Festa (field number 112);

SYNTYPE; MZUT Av7713 - skin; Greece, Rhodes, Koskino, 11 February 1913; collected by E. Festa (field number 8);

SYNTYPE; MZUT Av7714 - skin; Greece, Rhodes, Koskino, 11 February 1913; collected by E. Festa (field number 26).

Irania Finoti De Filippi, 1863

De Filippi F., 1863. Arch. Zool. Anat. Fisiol., vol. II, fasc. II: 381.

Now: Irania gutturalis (Guérin-Méneville, 1843)

HOLOTYPE; MZUT Av1993 - dismounted skin; Iran, 1862; collected by F. De Filippi.

Enicurus velatus Temminck, 1822

Temminck in Temminck C.-J. \& Laugier de Chartrouse M., 1822. Planches Coloriées, livr. 27, pl. 160.

Now: Enicurus velatus velatus Temminck, 1822

SYNTYPE; MZUT Av1230 - skin; male; Indonesia, Java, no date; collected by H. Kuhl and J.C. van Hasselt; from Temminck (Leiden Museum) in 1826;

SYNTYPE; MZUT Av1231 - skin; female; Indonesia, Java, no date; collected by H. Kuhl and J.C. van Hasselt; from Temminck (Leiden Museum) in 1826.

Notes: Dekker (2003: 41) quotes four syntypes in the Leiden Museum.

Arrenga melanura Salvadori, 1879

Salvadori T., 1879. Ann. Mus. Civ. Genova, vol. XIV: 227-228. 
Now: Myophonus melanurus (Salvadori, 1879)

SYNTYPE; MZUT Av9566 - dismounted skin; adult male; Indonesia, Sumatra, Singgalang Mt., 2 July 1878; collected by 0. Beccari; from G. Doria;

SYNTYPE; MZUT Av9567 - dismounted skin; adult male; Indonesia, Sumatra, Singgalang Mt., July 1878; collected by O. Beccari; from G. Doria.

Notes: Salvadori (1915: 29) reports as syntype only the specimen Av9566.

Pitta glaucina Temminck, 1823

Temminck in Temminck C.-J. \& Laugier de Chartrouse M., 1823. Planches Coloriées, livr. 33, pl. 194.

Now: Myophonus glaucinus (Temminck, 1823)

SYNTYPE (possible); MZUT Av659 - dismounted skin; adult male; Indonesia, Java, no date; from Temminck (Leiden Museum) in 1826.

Saxicola albo-marginata Salvadori, 1870

Salvadori T., 1870. Atti R. Acc. Sc. Torino, vol. V: 507-508.

Now: Oenanthe deserti deserti (Temminck, 1825)

HOLOTYPE; MZUT Av6597 - dismounted skin; adult male; Tunisia, Sahara, May 1866; collected by 0 . Antinori; from B. Comba.

Saxicola occidentalis Salvadori, 1887

Salvadori T., 1887. Ann. Mus. Civ. Genova, XXIII: 116-117.

Now: Oenanthe hispanica hispanica (Linnaeus, 1758)

SYNTYPE; MZUT Av1189 - mounted skin; adult male post-breeding; Italy, Genova, 10 October 1823; from M. Spinola collection;

SYNTYPE; MZUT Av1190 - mounted skin; adult male post-breeding; Italy, Genova, 3 September 1821; collected by A. della Marmora.

Note: Salvadori (1887a: 116) does not refer to any specimen, but some years later (Salvadori 1915: 33) he writes that n. 1189 and n. 1190 are to be considered type specimens.

Saxicola Brehmii Salvadori, 1870

Salvadori T., 1870. Atti R. Acc. Sc. Torino, vol. V: 508-509.

Now: Oenanthe lugubris schalowi (Fischer \& Reichenow, 1884)

HOLOTYPE; MZUT Av6972 - skin; adult male; Nubia? no date; from Verreaux, in 1867.

Saxicola albiscapulata Rüppell, 1837

Rüppell E., 1837. Neue Wirbelthiere zu der Fauna von Abyssinien gehörig. Vögel, pp. 7475, taf. 26, fig. 1.

Now: Thamnolaea cinnamomeiventris albiscapulata (Rüppell, 1837)

SYNTYPE; MZUT Av1219 - dismounted skin; female; Ethiopia, Abyssinia, March 1832; from E. Rüppell in 1839.

Notes: the specimen Av1219 bears two old labels: "Saxicola rufiventris / (Rüppell) / Abyssien (sic)" and "v. Rüppell / n. 214 / fömina / März 1832". It was certainly in the disposal of E. Rüppell, although with this temporary name, when in 1837 he described Saxicola albiscapulata, because the specimen was sent to the Museum of Torino only two years later. The identity of $S$. albiscapulata with $S$. rufiventris (binomen never described and known only as MS., as far as we know, only in the Museum of Torino), has been reported by Salvadori (1915: 10) and reaffirmed by Steinheimer (2005b: 191), but one cannot doubt that it was quite obvious for Rüppell himself. Rüppell most likely had forgotten to change the name on the label of the specimen sent to Turin: if he had thought it represented a new species, he would have described it together with the others Birds of Ethiopia and would not send the sole specimen to another museum! For these reasons we believe that the specimen Av1219 should be considered a syntype of S. albiscapulata, confirming the opinion of Salvadori (1915: 10) and Elter (1986: 421). 


\section{TURDIDAE}

Geocichla leucolaema Salvadori, 1892

Salvadori T., 1892. Ann. Mus. Civ. Genova, XXXII: 135-136.

Now: Geokichla interpres leucolaema (Salvadori, 1892)

SYNTYPE; MZUT Av17017 (former 4424); skin; adult; Indonesia, Enggano Is., 5 June 1891; collected by E. Modigliani (field number 135).

Notes: in the Genoa Museum there are two other syntypes (Arbocco et al. 1979: 223).

Turdus conradi Salvadori \& Festa, 1899

Salvadori T., Festa E., 1899. Boll. Mus. Zool. An. comp. Univ. Torino, No. 357 (vol. XIV): 4.

Now: Turdus chiguanco conradi Salvadori \& Festa, 1899

SYNTYPE; MZUT Av4488 - skin; adult male; Ecuador, between Narranjal and Cuenca; 9

October 1895; collected by E. Festa (field number 20);

SYNTYPE; MZUT Av4489 - skin; adult male; Ecuador, Cuenca, Sigsig; November 1895;

collected by E. Festa (field number 32);

SYNTYPE; MZUT Av4490 - skin; immature; Ecuador, Cuenca, August 1896; collected by

E. Festa (field number 517).

Notes: Elter (1986: 474) erroneously indicates the specimen Av4488 with the number 4480. The specimens Av4489 and Av4490 are not mentioned as types by Salvadori (1915: 38).

Turdus Werneri Gené, 1834

Gené G., 1834. Mem. R. Acc. Sc. Torino, T. XXXVII: 296-298, Pl. 2.

Now: Turdus obscurus Gmelin, 1789

SYNTYPE; MZUT Av581 - mounted skin; immature male; Italy, Piedmont, Leinì (near Turin), 4 November 1828.

Notes: Gené (1834: 296) wrote "Turdus Werneri Bon.", but F.A. Bonelli never described this species; his "new" name just appeared on the labels and on MS. catalogue, where there is a long and detailed description by Bonelli himself.

According to Salvadori (1915: 3) a second syntype was present at the Pisa Museum, but it was not possible for us to verify if this example still exists.

\section{APPENDIX 1 - SPECIMENS WITH NO TYPE STATUS}

The following are specimens that we do not consider as types, but which were reported, in some cases already in a dubitative form, as type-specimens (syntypes, types, cotypes, topotypes) by Salvadori (1915), by Elter (1986) or on the labels of the specimens.

The third and fourth editions of the International Code of Zoological Nomenclature (ICZN 1985,1999 ) define the topotype as "a term, not regulated by the Code, for a specimen originating from the type locality of the species or subspecies to which it is thought to belong, whether or not the specimen is part of the type series". For this reason, it seemed appropriate to check also the specimens that Salvadori (1915) has qualified in this way and that Elter (1986) has always treated as syntypes.

\section{CASUARIIDAE}

Dromaius ater Vieillot, 1817

Vieillot L. P., 1817, Nouveau dictionnaire d'Histoire Naturelle, X, pp. 212-13.

Now: Dromaius minor Spencer, 1906

MZUT Av2661; mounted skin; juvenile; Australia, King Island?, no date (but December 1802); collected during Nicolas Baudin's expedition. 
Notes: Salvadori (1915) in the index (page 45) indicates this specimen as "cotype?" but in the text (page 8) he does not consider it a typical specimen. Elter (1986: 186), perhaps due to the ambiguous indication of Salvadori, considers it a syntype. There are no elements that can link this specimen to the description of Vieillot (1817). Further details on this specimen are provided by Ghiraldi et Aimassi (2019: 30).

\section{ANATIDAE}

Nyroca australis Eyton, 1838

Eyton T. C., 1838. Monograph of the Anatidae. London, Longman, p. 160.

Now: Aythya australis (Eyton, 1838)

MZUT Av3570; mounted specimen; male; Australia, "Van Diemen's Land", 4 October 1838; from J. Gould.

Notes: Salvadori (1915: 13) and Elter (1986: 328) indicate this as syntype of Nyroca australis, but Salvadori attributes the species to Gould.

Eyton (1838: 160) described his species on a single specimen obtained from Gould, already associated with the MS. name Nyroca australis. Perhaps even the specimen Av3570, coming from Gould himself, had the same MS. name on the label, however we just found an old label reading "irides white".

\section{COLUMBIDAE}

Columba bitorquata Temminck, 1809

Temminck C.-J., 1810. Histoire naturelle générale des Pigeons. Livraison 9, p. 86, pl. 40.

Now: Streptopelia bitorquata (Temminck, 1809)

MZUT Av2466 - dismounted skin; Java, no date; collected by Kuhl \& Hasselt in 1820-21; from Temminck in 1826.

Notes: Av2466 was considered a type both by Salvadori (1915: 7) and Elter (1986: 152). According to Salvadori (1915: 7), this specimen was collected by Kuhl \& Hasselt, who were in Java in 1820-1821, so it cannot be a type of a species described in 1809.

Goura sclaterii Salvadori, 1876

Salvadori T., 1876. Ann. Mus. Civ. Genova, IX: 45-47.

Now: Goura scheepmakeri sclaterii Salvadori, 1876

MZUT Av9638; mounted skin; male; Papua New Guinea, Fly River, no date; collected by L.M. D’Albertis and donated by G. Doria.

Notes: the holotype, a female from Fly River hosted in the Museum of Genova (Arbocco et al. 1979: 197), is the only one specimen cited by Salvadori (1876c:45), but many others were captured subsequently in the same locality (Salvadori, 1882a: 199).

Elter (1986: 225), erroneously, indicates this specimen as syntype, but Salvadori (1915: 30) just quotes it as topotype.

Carpophaga consobrina Salvadori, 1887

Salvadori T., 1887. Ann. Mus. Civ. Genova, XXIV: 558-559.

Now: Ducula aenea consobrina (Salvadori, 1887)

MZUT Av11388; adult male; Indonesia, Mentawei, Si Oban, Li-pora, 24 April 1894; collected by E. Modigliani (field number 56).

Notes: quoted by Salvadori (1915: 36) and by Elter (1986: 117) as syntype, actually is not a type-specimen, because it was collected by Modigliani after the description of the species and not in the type-locality (Salvadori 1887b: 558, 1894c: 600).

Carpophaga chalconota Salvadori, 1874

Salvadori T., 1874. Ann. Mus. Civ. Genova, VI: 87-88.

Now: Ducula chalconota chalconota (Salvadori, 1874)

MZUT Av9207 - male; Indonesia, New Guinea, Bird's Head, Hatam, June 1875; collected by 0 . Beccari; from Genoa Museum. 
Notes: Elter (1986: 117), erroneously, indicates this specimen as syntype, but Salvadori (1915: 29) just quotes it as topotype. The holotype is hosted in the Museum of Genova (Arbocco et al. 1979: 194).

Columba lacernulata Temminck, 1822

Temminck in Temminck C.-J. \& Laugier de Chartrouse M., 1822, Planches Coloriées, livr. 28, pl. 194.

Now: Ducula lacernulata lacernulata (Temminck, 1822)

MZUT Av15374 - dismounted skin; immature; Indonesia, Java, no date; collected by Kuhl \& Hasselt in 1820-21; from Temminck in 1826.

Notes: the specimen Av15374 was considered a type either by Salvadori (1915:7) and by Elter (1986: 152). Actually, Temminck et Laugier (1822c) just refers to specimens collected by Reinwardt, so those coming from Kuhl \& Hasselt could not be type-specimens.

Ptilonopus bellus P. L. Sclater, 1874

Sclater P. L. 1874. Proc. Zool. Soc. London, XLIV [1873]: 696.

Now: Ptilinopus rivoli bellus P. L. Sclater, 1874

MZUT Av9309, skin; Indonesia, Atam, Monti Arfak, 1872; collected by L.M. D’Albertis; from Genoa Museum in 1876.

Notes: Salvadori (1915: 29) considers specimens Av9309 and Av9310 as "topotypes" of P. bellus and (page 31) the specimen Av6217 as "cotype"; Elter (1986: 395) considers the three specimens as syntypes. However, only Av9309 has the capture date and the locality that match the original description of Sclater (1874: 696).

Arbocco et al. (1979), according to Salvadori (1877a), report that in the Museo civico di Storia naturale di Genova there are some holotypes ("the types" for Salvadori) of the species collected by D'Albertis in Hatam, Arfak Mts., in 1872 and described by Sclater (1874). One of these is the holotype of P. bellus - C.E. 12135 (Arbocco et al. 1979: 193).

Since in the original description Sclater (1874: 696) did not indicate the number of specimens examined, and Salvadori (1915) and Elter (1986) considered the specimens of the MZUT as types, Aimassi et Ghiraldi (2017: 68) interpreted the indications by Salvadori (1877a) as lectotype designations (ICZN 1999, Art. 74.6). In this case, the skin Av9309 would be a paralectotype.

However, although it is unlikely that the number of specimens considered for each species can be identified with certainty, Sclater (1874: 690) examined 79 skins belonging to 53 species, so in many cases it is evident that he only examined one specimen. It also appears plausible that Salvadori knew well the specimens studied by Sclater: therefore, when he indicated "the type" he was really highlighting the holotypes, the only specimens that Sclater actually examined.

On the basis of these observations, we consider more appropriate to assume that the specimens of $P$. bellus hosted in the Turin museum do not have type status.

\section{APODIDAE}

Collocalia infuscata Salvadori, 1880

Salvadori T., 1880. Atti R. Acc. Sc. Torino, vol. XV: 348-349.

Now: Aerodramus infuscatus infuscatus (Salvadori, 1880)

MZUT Av2744 - skin; male; Indonesia, Ternate, 1 December 1874; collected by 0. Beccari. Notes: Salvadori (1915: 31) and Elter (1986: 151) consider Av2744 a type-specimen. The location and the date of capture coincide with those of the only one specimen described by Salvadori (1880c), which previously (Salvadori 1877c: 312) had been identified dubitatively as "Collocalia fuciphaga (Thunb.)" and referred to as "specimen unicum, in Ternate a Baccario collectum". Only afterwards Salvadori identified other three specimens of $C$. infuscata among those collected by Beccari in Ternate (Salvadori 1882a: 528, 1882b: 306, 1889c: 64). The Av2744 was part of a collection of 470 individuals, which 
was donated to the Turin Museum by the Marquis G. Doria in 1882 (Salvadori 1915: 31). The Holotype is hosted in the Museo Civico of Genoa (Arbocco et al. 1979: 205).

Cypelus horus von Heuglin, 1869

Heuglin M. T., 1869. Orn. Nordost-Afr., 1: 147.

Now: Apus horus (von Heuglin, 1869)

MZUT Av5536; male; Sudan, Sennar, November 1859; collected by O. Antinori (field number 289).

Notes: specimen Av5536 is indicated by Elter (1986: 174) as a syntype of "Cypselus horus Finsch et Hartlaub" but this is not correct.

Heuglin (1869b: 147-148), quotes “Cypselus horus, Hartl. \& Finsch, M. S.” as a var. of Cypselus affinis Gray, 1830: so, it makes the name C. horus available and becomes its Author. Salvadori et Antinori (1872), subsequently, provide a detailed description of this taxon, based on the specimen Av5536, which formerly Antinori (1864: 24) misidentified as Cypselus galilejensis.

\section{CUCULIDAE}

Diplopterus excellens P. L. Sclater, 1858

Sclater P. L., 1858. Proc. Zool. Soc. London, Part. XXV (N. 341): 229-230.

Now: Tapera naevia (Linnaeus, 1766)

MZUT Av349 - mounted skin; female; Mexico, no date; from Verreaux in 1861.

Notes: the Verreaux's label quotes "Diplopterus excellens Sclat. Proc. Zool. Soc. (1857) Mexique. (Type) female". A more recent label reads "Typus! / Messico / Dai Verreaux". Warren (1966: 93) list the holotype among the types of the Tring Museum. Despite the indications of the labels, neither Salvadori (1915) nor Elter (1986: 184) considered this specimen as a type. According to Sclater (1858: 229-230) the existence of a single type specimen cannot be proved, but the lack of precise capture data and the mere indication of "Type" on the label of Verreaux do not seem sufficient to qualify the specimen Av349 as syntype (paralectotype).

Chrysococcyx meyerii Salvadori, 1874

Salvadori T., 1874. Ann. Mus. Civ. Genova, VI: 82.

Now: Chalcites meyerii (Salvadori, 1874)

MZUT Av10171 - adult male; Arfak, no date; collected by A.A. Bruijn;

MZUT Av10172 - female; Mt. Arfak, Mori, 4 May 1875; collected by 0. Beccari.

Notes: Salvadori (1915: 31) and Elter (1876: 261) cited these specimens as types, however Salvadori could not introduce new types, since $C$. meyerii was a nomen novum for $C$. splendidus Meyer 1874. The holotype of $C$. meyerii Salvadori is the specimen described by Meyer, collected on “Arfakgebirge, Hattam circa 3500' hoch. Juli 1873" (Meyer, 1874: 82). Furthermore, Salvadori (1874c: 82) referred only to a specimen from Hatam, collected by L.M. D'Albertis. This bird is now hosted in the Genoa Museum and listed as "supporting specimen" (Arbocco et al. 1979: 250).

\section{CHARADRIIDAE}

Chettusia crassirostris Hartlaub, 1855

Hartlaub G., 1855, Journal für Ornithologie, V heft, N 17: 427.

Now: Vanellus crassirostris (Hartlaub, 1855)

MZUT Av5168 - dismounted skin; Sudan, White Nile, no date; collected by A. Brun-Rollet and sent to the Turin Museum in 1855;

MZUT Av5262 - mounted skin; Sudan, White Nile, no date; collected by A. Brun-Rollet and sent to the Turin Museum in 1855.

Notes: Salvadori (1915: 15) and Elter (1986: 177) report that these specimens are syn- 
types, but it is not correct. Certainly, Elter confuses the bibliographical reference, because she cites the work in which Salvadori (1866b) had described the new genus Defilippia and had indicated as a species-type "Defilippia crassirostris De Fil. in Hartl.".

Actually, Salvadori (1866b: 372, 1868b: 282) had already clarified the story of the specimens of Vanellus crassirostris that had appeared for the first time in Europe sent by Mr. A. Brun-Rollet to the Museum of Turin. Prof. Filippo De Filippi realized that they belonged to a new species and called it Chettusia crassirostris but he did not publish the description. However, he sent one of the specimens, associated with the name imposed by him, "to Mr. Verreaux of Paris", who in turn passed it to Hartlaub, who published the description for the first time. Hartlaub (1855: 427), kindly, associated the Latin binomial with the name of De Filippi, but obviously he remained as the unique author of the species.

The Turin specimens evidently have the same origin as the specimen described by Hartlaub, but they were never seen by him, so they are not type-specimens.

The holotype is located at the Übersee-Museum in Bremen, cat. UMB-9887 (Sanchez Osés 2010; M. Stiller, pers. comm. to G. Aimassi, 1 February 2016). The typical locality was specified by Grant et Mackworth-Praed (1936: 93).

\section{ACCIPITRIDAE}

Milvus affinis Gould, 1838

Gould J., 1838. Synopsis Birds Australia, Part III, Plate 47.

Now: Milvus migrans affinis Gould, 1838

MZUT Av3607 - mounted skin; Australia, Tasmania, Hobart Town, Clarence Plains, 29 October 1838; from Gould in 1842.

Notes: contrary to what Salvadori (1915: 13) and Elter (1986: 305) argued, specimen Av3607, collected on 29 October 1838, cannot be a type: the new species M. affinis was presented to the Zoological Society on December 26, 1837 (see PZS, part V, page 138) and the first description was published in April 1838 in the "Synopsis" (the date is according to M. Bruce 2011).

\section{PICIDAE}

Iyngipicus fusco-albidus Salvadori, 1874

Salvadori T., 1874. Ann. Mus. Civ. Genova, V: 42-43.

Now: Dendrocopos moluccensis moluccensis (J. F. Gmelin, 1788)

MZUT Av7017 - dismounted skin; female; Malaysia, Borneo, Sarawak, 5 August 1865; from G. Doria collection.

Notes: Salvadori (1915: 23) and Elter (1986: 259, but under the n. 1017) considered this specimen as a type, but in our opinion this interpretation is not correct.

Salvadori (1874), while introducing the new binomen Iyngipicus fusco-albidus, did not provide any description or definition of the new species, merely making a bibliographic reference to Wagler (1827), who had published a description. The name is valid according to Art. 12.2.1 of the Code (ICZN 1999), but the four specimens that Salvadori lists in introducing this species have no type status. The type-specimens should correspond to those examined by Wagler in the museums of Monaco and Paris ("Mus. Monac., Paris"). Furthermore, Salvadori (1874) believed he had introduced a nomen novum to replace "Picus variegatus, Wagl. (nec Lath.)", but there was no name to replace, because Wagler misidentified the specimens he was studying, without having introducing a new taxon.

\section{MOMOTIDAE}

Prionites martii Spix, 1824

von Spix J. B., 1824. Avium species novae, quas in itinere annis MDCCCXVII-MDCCCXX per Brasiliam [...], Vol. 1: 64-65. 
Now: Baryphthengus martii martii (Spix, 1824)

MZUT Av6617 - mounted skin; Costa Rica, 1868 or earlier; donated by Mr. Luigi Durando from Luserna.

Notes: Elter (1986: 482) marks this specimen with an asterisk, as if it were a type, however Salvadori (1915: 24) mentions the presence of the specimen Av6617 in the Turin Museum only because previously (Salvadori 1869b: 180-181) he designated Urospatha martii as a type-species of his new genus Urospatha.

\section{ALCEDINIDAE}

Tanysiptera galatea var. minor Salvadori \& D'Albertis, 1875

Salvadori T. \& L.M. D’Albertis, 1875. Ann. Mus. Civ. Genova, VII: 815.

Now: Tanysiptera galatea minor Salvadori \& D'Albertis, 1875

MZUT Av9579 - adult male; Papua New Guinea, Fly River, 14 October 1877; collected by L.M. D'Albertis (field number 742); donated by G. Doria.

Notes: Salvadori (1915: 30) includes this specimen in a list of topotypes, but Elter (1986: 454) refers to it as a syntype. Actually, specimen Av9579 is not a type, because it was collected after the description of the species.

Four syntypes are hosted in the Museo Civico di Storia naturale di Genova (Arbocco et al. 1979: 207).

\section{FALCONIDAE}

Falco eleonorae Gené, 1839

Gené G. [in Anonymous], 1839. Revue Zoologique, II: 105.

Now: Falco eleonorae Gené, 1839

MZUT Av49 - mounted skin; female (pale morph); Italy, Sardinia, Toro Is., 22 August 1839; collected by A. della Marmora;

MZUT Av51 - mounted skin; female (dark morph); Italy, Sardinia, Vacca Is., 20 August 1839; collected by A. della Marmora.

Notes: Salvadori (1915: 11) claims that the first description of Falco eleonorae occurred in the Revue Zoologique (Gené 1839: 105) and indicates the specimens Av49 and Av51 as types. Previously Salvadori himself (1864: 149) had supported a similar opinion, but he believed that the description was to refer to a different paper (Gené 1840), published in the Memorie della Reale Accademia delle Scienze di Torino. Elter (1986: 205) also considers the specimens of Turin as syntypes and indicates as the first description the one published on the Memorie (Gené 1840).

Although the description published in the Memorie is very detailed and is accompanied by two colour plates, the priority must be accorded to the brief note of an anonymous Author published in the Revue Zoologique, which shows the few but most significant words of the description, proposed by Gené in the session of the Accademia delle Scienze di Torino, March 3,1839 .

Only by reading the text that appeared in the Memorie is it possible to know that Gené proposed the new species Falco eleonorae describing two females that had been captured in 1838 by Antonio della Marmora in Sardinia, on the Toro Island. So, the specimens of the Turin Museum, captured in August 1839, cannot be types.

The text of the Gené paper is on pp. 41-47 of the Tome II (Series II) of the Memorie. On p. 48 Gené has included a note, dated September 1839, where he proposed some new observations and corrections, based on new specimens captured by Marmora, including almost certainly those still preserved in the MZUT collection.

In the same note, Marmora wrote that pages $41-47$ had already been printed, so we could assume that the date of publication of this part of the Memorie is earlier. However, this is not a definite proof, since pages 41 to 48 are printed in the same gathering (eight pages "in quarto"), signed "F". 


\section{PSITTACULIDAE}

Lorius erythrothorax Salvadori, 1877

Salvadori T., 1877. Ann. Mus. Civ. Genova, X: 32.

Now: Lorius lory erythrothorax Salvadori, 1877

MZUT Av9621 - skin; female; Papua New Guinea, Fly River, June 1876; collected by L.M. D’Albertis; donated by G. Doria.

Notes: specimen MZUT Av9621 has been indicated by Salvadori (1915: 30) as a topotype and Elter (1986: 281) referred to it as syntype. This is not correct, because Salvadori (1877b: 32) described L. erythrothorax on the basis of a single specimen, captured at Mount Epa, previously identified as Lorius lory (Salvadori et D’Albertis 1875: 812).

The holotype was housed in the Genoa museum, but it has not been found by Arbocco et al. (1979: 197).

Chalcopsittacus chloropterus Salvadori, 1876

Salvadori T., 1876. Ann. Mus. Civ. Genova, IX: 15-16.

Now: Chalcopsitta scintillata chloroptera Salvadori, 1876

MZUT Av9620 - skin; female; Papua New Guinea, Fly River, 8 June 1877; collected by L.M. D’Albertis; donated by G. Doria.

Notes: Salvadori (1915: 30) lists the MZUT Av9620 specimen in a series of duplicates obtained in 1979 from the Genoa Museum, but in the work index (page 44) he indicates it as a topotype. According to Elter (1986: 127) it would be a syntype.

Salvadori (1880b: 276) considers this species as junior synonym of Chalcopsittacus scintillatus (Temminck) and cites a list of specimens, but the only one with date 8 June 1877 is the female indicated with the letter b8, N. 80 of the Collection D'Albertis, which consequently corresponds to the specimen Av9620. In the same work Salvadori recalls that the Holotype of Chalcopsittacus chloropterus was captured at the Baja Hall in September 1875 and corresponds to the male n. 444 of the D'Albertis Collection. This specimen is housed in the Genoa Museum (Arbocco et al. 1979: 197): the capture site is the Yule Island, located near Baja Hall.

Cyclopsittacus cervicalis Salvadori \& D’Albertis, 1876

Salvadori T. \& L. M. D’Albertis, 1876. Ann. Mus. Civ. Genova, VII: 811-812.

Now: Psittaculirostris desmarestii cervicalis (Salvadori \& D’Albertis, 1876)

MZUT Av9577 - male; Papua New Guinea, Fly River, 7 September 1877; collected by L.M. D’Albertis (field number 593).

Notes: Salvadori (1915: 30) lists this specimen among a series of duplicates obtained in 1979 from the Genoa Museum and in the work index (page 45) he indicates it as a topotype. Elter (1986: 172) deals with the same specimen as syntype. In fact, Av9620 does not belong to the typical series because it was collected after the description of the species.

The capture site is specified only in Salvadori (1880b:156), where the specimen is listed with the letter "e". The three syntypes are hosted in the Genoa Museum (Arbocco etal.1979: 198). Dickinson et Remsen (2013: 383) and del Hoyo et Collar (2014: 748) indicate 1876 as the description date for C. cervicalis, but Poggi (2010: 346) had suggested "XII.1875".

Cyclopsittacus fuscifrons Salvadori, 1876

Salvadori T., 1876. Ann. Mus. Civ. Genova, IX: 14-15.

Now: Cyclopsitta melanogenia fuscifrons (Salvadori, 1876)

MZUT Av9618 - male; Papua New Guinea, Fly River, 4 August 1877; collected by L.M. D'Albertis (field number 416);

MZUT Av9619 - female; Papua New Guinea, 4 August 1877; collected by L.M. D’Albertis (field number 414).

Notes: Salvadori (1915: 30) lists the specimens Av9618 and Av9619 among a series of duplicates obtained in 1979 from the Genoa Museum and in the work index (page 45) indicates them as topotypes. According to Elter (1986: 172) they would be syntypes. Actually, these specimens do not belong to the typical series because they were collected 
two years after those mentioned in the description of $C$. fuscifrons.

Salvadori (1880b: 168) considered that only the specimen "i", a male collected in December 1875, Fly River, was "the type" of Cyclopsittacus fuscifrons, so designating it as Lectotype. This specimen is housed in the Museum of Genoa, where Arbocco et al. (1979: 199) listed 3 syntypes.

Cyclopsitta aruensis Salvadori, 1874

Salvadori T., 1874. Ann. Mus. Civ. Genova, VI: 73-75.

Now: Cyclopsitta diophthalma aruensis (Schlegel, 1874)

MZUT Av9616 - male; Papua New Guinea, Fly River, no date; collected by L.M. D'Albertis; MZUT Av9617 - female; Papua New Guinea, Fly River, no date; collected by L.M. D’Albertis. Notes: Salvadori (1915: 30) quotes these specimens as topotypes and Elter (1986: 172) as syntypes. The types were collected by Beccari in the Aru Islands (Salvadori 1874b: 7475, 1880b: 161-162); the specimens of the Turin Museum were collected by D'Albertis in a different site, probably in 1877 (Salvadori 1880b: 162), so they are not the types.

The two syntypes collected by Beccari are still housed in the Genoa Museum (Arbocco et al. 1979: 199).

\section{CLIMACTERIDAE}

Climacteris picumnus Temminck, 1824

Temminck in Temminck C.-J. \& Laugier de Chartrouse M., 1824, Planches Coloriées, livr. 47, pl. 281, fig. 1 .

Now: Climacteris picumnus picumnus Temminck, 1824

MZUT Av3616 - female? South Australia, date not readable; donated by J. Gould in 1842 or 1843.

Notes: Salvadori (1915: 13) listed the Av3616 specimen among those that J. Gould had sent to the Turin Museum in the years 1842-43. Perhaps for this reason Salvadori erroneously attributed C. picumnus to Gould and indicated the specimen Av3616 as "Cotype"; Elter (1986: 147) also repeated the same error. Actually, the species was described by Temminck et Laugier (1824a) and the Turin specimen is not involved.

\section{MELIPHAGIDAE}

Ptilotis cinerea P. L. Sclater, 1874

Sclater P. L., 1874. Proc. Zool. Soc. London, [1873, N. XLIV]: 693.

Now: Pycnopygius cinereus cinereus (P. L. Sclater, 1874)

MZUT Av4126 - skin; female; Indonesia, Hatam, 18 September 1872; collected by L.M. D'Albertis.

Notes: in the original description Sclater (1874: 693) does not indicate the number of specimens studied, but Salvadori (1877a: 145) signals as "the type" a male from Hatam (field number 319) and reports that among the specimens collected by D'Albertis there was also a female, which evidently corresponds to the MZUT specimen Av4126. Arbocco et al. (1979: 240) indicate the presence of the holotype in the Genoa museum. The skin Av4126 was not mentioned by Salvadori (1915) and Elter (1986: 397) did not indicate it as a type.

For a discussion on the status of this specimen see also the notes under Ptilinopus bellus.

Melipotes gymnops P. L. Sclater, 1874

Sclater P. L., 1874. Proc. Zool. Soc. London, [1873]: 695-696.

Now: Melipotes gymnops P. L. Sclater, 1874

MZUT Av4098 - skin; male; Indonesia, Papua New Guinea, Hatam, 23 September 1872; collected by L.M. D'Albertis (field number 483);

MZUT Av4099 - skin; Indonesia, Papua New Guinea, Hatam, 23 September 1872; collected by L.M. D'Albertis.

Notes: in the original description Sclater (1874: 695-696) does not indicate the number of skins 
studied, but Salvadori (1877a: 145) signals 3 specimens collected by D'Albertis in Hatam, one of which is "the type". Arbocco et al. (1979: 241) report the presence of the holotype at the Genoa museum (C.E. 11394). However, specimens Av4098 and Av4099 are treated as types by Salvadori (1915: 31) and Elter (1986: 293).

For a discussion on the status of these specimens see also the notes under Ptilinopus bellus.

Philemon meyeri Salvadori, 1878

Salvadori T., 1878. Ann. Mus. Civ. Genova, XII: 339.

Now: Philemon meyeri Salvadori, 1878

MZUT Av9586 - adult female; Papua New Guinea, Fly River, 9 or 11 June 1877; collected by L.M. D'Albertis (field number 97).

Note: the MZUT Av9586, cited in Salvadori (1881b: 350) with the letter "e", was indicated by Salvadori (1915: 30) as topotype and by Elter (1986: 360) as syntype. However, the type-specimens of P. meyeri were collected by Meyer and Bruijn (Salvadori 1878d: 339) in different localities.

Tropidorhynchus citreogularis Gould, 1837

Gould J., 1837. Synopsis Birds Australia, Part I, Plate 13, Fig. 1.

Now: Philemon citreogularis citreogularis (Gould, 1837)

MZUT Av3551 - dismounted skin; adult female; Australia, Port Essington, 19 September 1840; donated by J. Gould in 1842 .

Notes: this specimen cannot be a type because the capture date indicated on the label is more recent than the original description. Salvadori (1915: 13) and Elter (1986: 472) are in error.

\section{CAMPEPHAGIDAE}

Ceblepyris marginatus Temminck MS.

Never described.

Now: Coracina javensis (Horsfield, 1821)

MZUT Av783 - female; Indonesia, Java, no date; from C.J. Temminck (Leiden Museum).

Notes: Temminck donated to the Turin museum the specimen Av783, already labelled as Ceblepyris marginatus, but he never described this species, as we deduce from Salvadori (1915: 7), for which it corresponds to Coracina javensis (Horsfield, 1821). Also Pelzeln (1865:

80) had reported a specimen in the "kaiserlichen Museum" of Vienna, with the Temminck MS. name "Ceblepyris marginatum fem. sp. nov.", which he identified as "Graucalus javensis (Horsf.)?”.

Elter (1986: 120) indicated the specimen Av783 as a syntype of Ceblepyris marginatus Temminck, but she referred to Horsfield (1821) for the description paper.

Ceblepyris papuensis Temminck MS.

Never described.

Now: Coracina javensis (Horsfield, 1821)

MZUT Av787 - male; Indonesia, Java, no date; collected by Kuhl \& Hasselt in 1820-21; from Temminck in 1826;

MZUT Av788 - male; Indonesia, Java, no date; collected by Kuhl \& Hasselt in 1820-21; from Temminck in 1826.

Notes: Salvadori (1915: 7) lists these specimens in a series of "types, or cotypes" that the Turin Museum received from Temminck, but actually reports a misidentification, because he writes "Ceblepyris papuensis Temm. (nec Gm.)" and states that they must be identified as Graucalus javensis (Horsfield). Elter (1986: 120) indicated the specimens Av787 and Av788 as syntypes of Ceblepyris papuensis Temminck, and referred to Jerdon (1839: 242) for the description paper.

As far as we now, Temminck never described a Ceblepyris papuensis.

Edoliisoma müllerii Salvadori, 1876

Salvadori T., 1876. Ann. Mus. Civ. Genova, VII: 927-928. 
Now: Edolisoma tenuirostre muellerii Salvadori, 1876

MZUT Av15917 (former 3126) - skin; immature female; Indonesia, Aru Is., Wokam, 29 May 1873; collected by 0. Beccari (field number 435).

Notes: Salvadori (1876: 927-8) introduced the binomen Edoliisoma müllerii explicitly to replace Ceblepyris plumbea S. Müller, which he considered preoccupied by C. plumbea Wagler. However, Salvadori (1876) also provided a short definition and claimed to have examined some specimens collected by Beccari and Bruijn. Their capture data were specified a few years later by the same author (Salvadori 1881b: 154-155), who quoted specimen Av15917 with the letter "h".

Since Edoliisoma müllerii is a nomen novum, the types must coincide with those associated with C. plumbea S. Müller (ICZN 1999, art. 72.7). The Genoa Museum houses six of the specimens cited by Salvadori, which Arbocco et al. (1979: 250) do not consider typespecimens but simply as "supporting specimens".

On the contrary, Salvadori (1915: 31) and Elter (1986: 188) quoted the specimen of the Turin Museum No. 3126 (now Av15917) as a type.

\section{CINCLOSOMATIDAE}

Eupetes leucostictus P. L. Sclater, 1874

Sclater P.L. 1874. Proc. Zool. Soc. London, for 1873: 690-691, Pl. LII.

Now: Ptilorrhoa leucosticta leucosticta (P. L. Sclater, 1874)

MZUT Av4339 - male; Indonesia, New Guinea, Bird's Head, Hatam, 26 June 1875; collected by 0 . Beccari; from G. Doria in 1883.

Notes: Av4339 is not a type, because it was collected after the publication of Sclater (1874), who described only a specimen "obtained by Signor d'Albertis at Atam in October 1872". The holotype is hosted in the Genoa Museum (Arbocco et al. 1979: 214).

Salvadori (1915: 31) and Elter (1986: 201) erroneously consider this specimen as a type.

\section{PACHYCEPHALIDAE}

Pachycephala soror P. L. Sclater, 1874

Sclater P. L., 1874. Proc. Zool. Soc. London, for 1873: 692.

Now: Pachycephala soror soror P. L. Sclater, 1874

MZUT Av3363 - skin; male; Indonesia, Hatam, Arfak, September 1872; collected by L.M. D'Albertis.

Notes: Salvadori (1915: 31) and Elter (1986: 339) consider this specimen as a syntype, whose capture data match the original description of Sclater (1874: 692). However, according to Arbocco et al. (1979: 229), the holotype is housed in the Genoa museum. For a discussion on the status of this specimen see the notes under Ptilinopus bellus.

Salvadori (1915: 31) and Elter (1986: 339) also consider the MZUT skins Av3364 and Av3365 as type specimens, but they were collected in 1875, after the description of the species.

Hyloterpe philomela Temminck MS.

Never described.

Now: Pachycephala cinerea cinerea (Blyth, 1847)

MZUT Av929 - dismounted specimen; Indonesia, Java, no date; collected by Kuhl \& Hasselt in 1820-21; from Temminck in 1826.

Notes: Salvadori (1915: 7), listing the specimen Av929 in a series of "types, or cotypes" that the Turin Museum received from Temminck, states that "Muscicapa philomela Temm. Mus. Taur. et Berol." corresponds to Pachycephala grisola Blyth; the same synonymy is suggested by Blyth himself "in epist." (Jerdon 1864: 871). Walters (2003) stated that the correct name to be adopted is Pachycephala cinerea (Blyth).

It seems evident that the binomen Muscicapa philomela was never described by Temminck, who limited himself to sending specimens labelled with this name to the Museums of Turin 
and Berlin. The specimens of Berlin are also mentioned by Bonaparte (1850: 329) and Gadow (1883: 220).

Elter (1986: 312) is wrong in considering Av929 as a type specimen.

\section{OREOICIDAE}

Pachycephala rufinucha P. L. Sclater, 1874

Sclater P. L., 1874. Proc. Zool. Soc. London, XLIV [1873]: 692.

Now: Aleadryas rufinucha rufinucha (P.L. Sclater, 1874)

MZUT Av3369 - skin; male?; Indonesia, Hatam, Arfak, 19 September 1872; collected by L.M. D'Albertis.

Notes: Sclater (1874: 692) did not specify the number of specimens studied, but it seems likely that he saw only one skin of this species (see notes under Ptilinopus bellus). Although Salvadori (1915: 31) and Elter (1986: 338) treat the Av3369 specimen as a syntype, Salvadori (1877a: 141) and Salvadori (1881b: 225) had indicated a different specimen as "the type".

\section{DICRURIDAE}

Buchanga longa Temminck MS.

Never described.

Now: Dicrurus macrocercus Vieillot, 1817

MZUT Av778 - dismounted specimen; unsexed; Indonesia, Java, no date; from Temminck (Leyden Museum).

Notes: Salvadori (1915: 7), listing the specimen Av778 in a series of "types, or cotypes" that the Turin Museum received from Temminck, states that "Edolius longus Temm. MS." corresponds to Dicrurus macrocercus Vieillot.

According to van Oort (1910: 151), the "type" of Temminck "upon which is based the 14th species of Dicrourus in Bonaparte's Conspectus Avium [...] is still in the collection of the Leyden Museum". The specimen was donated by Temminck to the Turin Museum before 1830, so we can exclude that it may be a type of Dicrourus longus Bonaparte (1850: 352). Elter (1986: 188) quotes the specimen Av778 as Edolius longus Temminck and marks it with an asterisk, as if it were a type. However, in a footnote she states "Temminck, M.S."

\section{RHIPIDURIDAE}

Rhipidura leucothorax Salvadori 1874

Salvadori T., 1874. Ann. Mus. Civ. Genova, VI: 311-312.

Now: Rhipidura leucothorax leucothorax Salvadori, 1874

MZUT Av2983 - skin; male; Papua New Guinea, Naiabui, 1875; collected by L.M. D’Albertis (field number 569).

Notes: Salvadori (1915: 31) and Elter (1986: 411) erroneously consider this specimen as a type, but actually it was collected after the description of the species. In Salvadori (1881b: 58) this specimen is listed under letter " $\mathrm{e}$ ".

\section{CORVIDAE}

? Glaucopis varians "Temminck"

Never described.

Now: Crypsirina temia (Daudin, 1800)

MZUT Av369 - male; Indonesia, Java, no date; collected by Kuhl \& Hasselt in 1820-21; from Temminck in 1826.

Notes: Salvadori (1915: 7) and Elter (1986: 224) treat the specimen as a type of a species described by Temminck in the Planches coloriées. Actually Temminck, in $57^{\mathrm{me}}$ livr., in the text of "Genre Glaucope" lists four species and the $4^{\text {th }}$ is Glaucope temmia Glaucopis varians, illustrated by Le Vaillant with the name "Temia" and described from Latham as 
Corvus varians. So Temminck never described a "Glaucopis varians".

The specimen described and figured by Levaillant (1800: 22-23, Pl. 56), to which the descriptions of Corvus temia Daudin and Corvus varians Latham refer, was part of the Temminck collection and is no longer present at the Leiden Museum (Dekker et Quaisser 2006: 69). However, if the Turin specimen comes from the Kuhl-Hasselt expedition, as reported in the MS. Catalogue of F. A. Bonelli and in Salvadori (1915: 7), it cannot be a type of Daudin or Latham.

\section{MONARCHIDAE}

Monarcha godeffroyi Hartlaub, 1868

Hartlaub G., 1868. Proc. Zool. Soc. London, for 1867: 829.

Now: Metabolus godeffroyi (Hartlaub, 1868)

MZUT Av8058 - unsexed specimen; Yap State (Federated States of Micronesia), Yap island, 1870; from Godeffroy Museum.

Notes: the specimen has a label of the Godeffroy Museum, Hamburg, No. 4053, where we can also read 89 / 1870; a second label, more recent, reads that the bird was collected by Kubary in Uap Is. and reports “Cotypus!". Elter (1986: 309) considers the specimen MZUT Av8058 as a type, but Salvadori (1915) does not mention it.

Hartlaub et Finsch (1872: 97) quoted eleven specimens of "Monarches godeffroyi" collected by J. Kubary (Bolau 1898: 56), stated that the Kubary specimens were collected in 1870 and that they are not at all typical.

In the Übersee-Museum Bremen, Sànchez Osés (2010: 58) indicates the presence of the holotype, with catalogue number 8498 , but actually it is a syntype, because Hartlaub (1868: 829) explicitly described 3 specimens "shot in the island of Yap".

\section{PARADISAEIDAE}

Drepanornis albertisi Sclater, 1873

Sclater P.L., 1873. Nature, 8 (August, 14): 305-306.

Now: Drepanornis albertisi albertisi P.L. Sclater, 1873

MZUT Av8833 - skin; female; Indonesia, Bird's Head, NW New Guinea, Andai, 30 November 1875; collected by O. Beccari;

MZUT Av8832 - male; Indonesia, Bird's Head, NW New Guinea, Hatam, 1.7.1875; collected by 0 . Beccari.

Notes: Salvadori (1915: 29) and Elter (1986: 186) consider these specimens as types, however Sclater described two birds collected by L.M. D'Albertis, while the specimens of the Turin Museum were collected by 0 . Beccari two years after the description. The syntypes are hosted in the Genoa Museum (Arbocco et al. 1979: 248).

Even in a Richmond card (http://www.zoonomen.net/cit/RI/SP/Didu/didu00380a.jpg, accessed 16 December 2018) the 8832 and 8833 specimens of the Turin Museum are erroneously referred to as types.

Dickinson et Christidis (2014: 252) and del Hoyo et Collar (2016: 364), according to Mayr et Greenway (1962: 189), insert the author's name in brackets because they consider that the species has been described as Drepanephorus albertisi in Nature's issue of June 19th, on p. 151. However, on that date Sclater did not provide any description of the species and in our opinion the first valid name is that published, again on n. 8 of Nature, but in the issue of August 14 on p. 305, where the name Drepanornis albertisi already appears. Despite the research, we could not find the specimen Av8832 in the MZUT collection.

\section{PETROICIDAE}

Megalestes albonotatus Salvadori, 1875

Salvadori T., 1876. Ann. Mus. Civ. Genova, VII: 770.

Journal of the National Museum (Prague), Natural History Series | 2020 | 133 
Now: Plesiodryas albonotata albonotata (Salvadori, 1875)

MZUT Av2940 - skin; female; Indonesia, Bird's Head, NW New Guinea, Arfak Mts., 8 April 1875; collected by A.A. Bruijn.

Notes: Salvadori (1915: 31) and Elter (1986: 288) consider this specimen as a type but actually the holotype is hosted in the Genoa Museum (C.E. 14483, Arbocco et al. 1979: 225).

An old label states: "d. Megalestes albonotatus, Salvad. Similar to the type. Bruijn". The specimen "d" is mentioned in Salvadori (1881b: 82 ) with a slightly different date: 28 April 1875.

Leucophantes brachyurus P. L. Scalter, 1874

Sclater P.L. 1874. Proc. Zool. Soc. London, for 1873: 692.

Now: Poecilodryas brachyura brachyura (P.L. Sclater, 1874)

MZUT Av2937 - skin; male; Indonesia, Bird's Head, NW New Guinea, Andai, 26 May 1875; collected by A.A. Bruijn; from O. Beccari.

Notes: Salvadori (1915: 31) and Elter (1986: 378) consider this specimen as a type, however the syntypes of Sclater were collected by L. M. d'Albertis and the specimen Av2937 was collected by A.A. Bruijn after the description of the species. The types and the specimen of the MZUT collection are easily identifiable in Salvadori (1877a: 133-134, 1881b: 87).

Myiolestes? cyanus Salvadori, 1874

Salvadori T., 1874. Ann. Mus. Civ. Genova, VI: 84.

Now: Peneothello cyanus cyanus (Salvadori, 1874)

MZUT Av2936 - skin; female; Indonesia, Bird's Head, NW New Guinea, Arfak Mts., 1 May 1875; collected by 0 . Beccari.

Notes: Salvadori (1915: 31) and Elter (1986: 378) consider this specimen as a type, however it was collected after the description of the species.

Myiolestes? bimaculatus Salvadori, 1874

Salvadori T., 1874. Ann. Mus. Civ. Genova, VI: 84.

Now: Peneothello bimaculata bimaculata (Salvadori, 1874)

MZUT Av2939 - skin; male; Indonesia, Bird's Head, NW New Guinea, Arfak Mts., "Profi”, 9 July 1875; collected by 0 . Beccari.

Notes: Salvadori (1915: 31) and Elter (1986: 378) consider this specimen as a type, however it was collected after the description of the species.

\section{NECTARINIIDAE}

? Nectarinia lepida "Temminck"

Never described.

Now: Anthreptes malacensis malacensis (Scopoli, 1786)

MZUT Av2217 - adult male; Java, no date; collected by H. Kuhl \& J.C. van Hasselt; from Temminck (Leiden Museum);

MZUT Av2218 - adult female; Java, no date; collected by H. Kuhl \& J.C. van Hasselt; from Temminck (Leiden Museum).

Notes: Salvadori (1915: 8) considers these specimens as types of Nectarinia lepida and Elter (1986: 319) also states that this species was described by Temminck in the Pl. Col. 126. Actually, in this Planche, Temminck "describes" the male and the female of the species Souimanga gracieux Nectarinia lepida but is says explicitly that "C'est la Certhia lepida Lath., Ind. Orn., vol. 1, p. 298, sp. 60". So, it is not a new species.

Despite the research, we could not find the specimen Av2217 in the MZUT collection.

\section{EMBERIZIDAE}

Emberiza intermedia "de Michahelles" Degland, 1849

Degland C. D., 1849. Ornithologie européenne. Tome I. Paris, p. 264. 
Now: Schoeniclus schoeniclus intermedius (Degland, 1849)

MZUT Av1398 - dismounted skin; adult male, winter plumage; Dalmatia, no date; obtained from Michahelles in 1832.

Notes: the species was never described by Michahelles, although in a work on Dalmatian vertebrates he discussed about an Emberiza with comparable characters both to the "schoeniclus" specimens of central Germany and to the "palustris" of Tuscany (Italy). The same notice was in Savi (1831: 225-6): "Dr Michaellis (sic) of Nuremberg wrote to me that on a journey he made in Dalmatia he saw a species whose beak has intermediate shape and dimensions between that of schoeniclus and that of palustris ".

Degland (1849: 264) provided some elements to describe E. intermedia "de Michahelles" and, for this, is considered the true author of the species although, as far as we know, this was noticed only long afterwards by Steinbacher (1930).

Since Degland does not refer to any specimen in particular and we do not know any sources from which we can deduce which specimens were studied by him, we believe that there are no type-specimens of E. intermedia. For this reason, contrary to what was stated by Salvadori (1915: 10) and Elter (1986: 192), the MZUT Av1398 specimen, despite being collected by Michahelles in Dalmatia, cannot be considered as a type. We believe it is possible that this specimen was collected by Michahelles together with those now hosted in the American Museum of Natural History "from the Brehm Collection via the Rothschild Collection", a lectotype and a paralectotype of Cynchramus canneti Brehm (LeCroy 2012: 9).

\section{PYCNONOTIDAE}

\section{? Muscicapa psidii "Temminck"}

Never described by Temminck.

Now: Pycnonotus goiavier analis (Horsfield, 1821)

MZUT Av629 - unsexed specimen, Indonesia, Java, no date; collected by H. Kuhl and J.C. van Hasselt (1820-21); from Temminck in 1826.

Notes: Salvadori (1915: 7) and Elter (1986: 312) consider this specimen as a type, however this name is not associated with any description of Temminck and its indication is the result of a misidentification. Muscicapa psidii was described by Gmelin (1789: 941).

\section{SYLVIIDAE}

Drymoeca (?) pekinensis Swinhoe, 1868

Swinhoe R., 1868. Ibis, n.s., vol. IV: 61-62.

Now: Rhopophilus pekinensis pekinensis (Swinhoe, 1868)

MZUT Av6308 - unsexed specimen; China, Beijing, 15 June 1864; donated by A. David and M. Fontanier in 1866, during "Magenta" journey.

Notes: the specimen Av6308 is quoted by Elter (1986: 412, as Rhopophilus pekinensis) as a syntype, however the bibliographic reference is erroneous, referring to the description of Cypselus pekinensis Swinhoe 1870. Salvadori (1915: 21) just remember that Rhopophilus pekinensis and Pterorhinus davidi (he did not refer to any specimens) were sent by Prof. De Filippi to the Turin Museum in 1867, in a collection of 232 specimens obtained during the journey of the Magenta and that they were described some time later. No specimens of $P$. davidi are currently present in the Turin Museum.

Giglioli et Salvadori (1870b: 187) point out that when the two birds were received, they were "at once perceived to be new" by Prof. De Filippi and Giglioli. Furthermore, "Mr. Swinhoe, who was communicated with on the subject, could not identify either". But Swinhoe himself (1868: 60-63), when he received two further specimens from Father David, described the new species Drymoeca (?) pekinensis and Pterorhinus davidi, without making any reference to the specimens previously seen. Giglioli et Salvadori (1870b: 187 ) introduced the new genus Rhopophilus, with type-species R. pekinensis! 


\section{TIMALIIDAE}

Mixornis flavicollis Bonaparte, 1850

Bonaparte C., 1850. Conspectus Generum Avium, vol. 1, Lugduni Batavorum, pag. 217.

Now: Mixornis flavicollis flavicollis Bonaparte, 1850

MZUT Av673 - skin; Indonesia, Java, no date; collected by H. Kuhl and J.C. van Hasselt in 1820-21; from Temminck in 1826.

Notes: one side of an old label reads "673. Timalia / Myiothera fulvicollis (sic!), Kuhl et / Giava (K. et v. H.) / from Temminck"; the other one "Mixornis (Timalia) / flavicollis, Salomon Müller. Bp. / Consp. p. 217".

According to Elter (1986: 307) this specimen would be a syntype and, remarkably, he has the same provenance as one of the four syntypes still present in Leiden (Dekker 2003: 49). Bonaparte (1850: 217) described Mixornis flavicollis on the basis of the specimens present in Leiden, named "Timalia flavicollis Müll.", however the specimen Av673 was at the Turin Museum since 1826, so Bonaparte could not have seen it in Leiden.

\section{PELLORNEIDAE}

Myiothera pica Temminck MS. (or Boie MS.)

Never described.

Now: Melanocincla sepiaria sepiaria (Horsfield, 1821)

MZUT Av664 - Indonesia, Java, no date; collected by H. Kuhl and J.C. van Hasselt in 182021; from Temminck in 1826.

Notes: Salvadori (1915: 8) wrote that the name "Myiothera pica vel pyca" corresponded to a manuscript indication of Temminck and proposed the "synonymy" with "Turdinus sepiarius Horsf.". However, in the same paper (page 46, in index), he defined the specimen Av664 as a cotype of Temminck. Elter (1986: 316) considered this specimen as a syntype of Myiothera pica Temminck, but referred to the work of Horsfield (1821: 158) who described Brachypteryx sepiaria. The specimens studied by Horsfield were part of a collection of Java birds, collected in the years 1811-1817 and housed in the Museum of the Honourable East India Company (Horsfield 1822: 133); two syntypes are now kept at the Tring Museum (Warren et Harrison 1971: 502). The description of B. sepiaria is very brief and there is no reference, neither bibliographic nor to specimens present in other collections. Therefore, the Av664 specimen is neither a type of B. sepiaria, nor of Myiothera pica, a species never described by Temminck.

\section{SITTIDAE}

Sitta melanocephala Temminck MS.

Never described.

Now: Sitta azurea azurea Lesson, 1830

MZUT Av2073 - male; Indonesia, Java, no date; collected by H. Kuhl and J.C. van Hasselt in 1820-21; from Temminck in 1826.

Notes: Salvadori (1815: 7) wrote that the name Sitta melanocephala corresponded to a manuscript indication of Temminck and proposed the "synonymy" with "Sitta azurea Less.". However, in the same work (page 46, in index), he defined the specimen Av2073 as a cotype of Temminck.

Elter (1986: 430) considered this specimen as a syntype of "Sitta melanocephala Temminck", but she referred to Lesson (1830: 316), who described Sitta azurea. This description is very short and there is no reference to the Turin specimen, so Av2073 is not a syntype.

\section{MUSCICAPIDAE}

Ruticilla fuscicaudata (Blanford, 1869)

Blanford W.T., 1869, Ann. Mag. Nat. Hist., Ser. 4, Vol. IV, No. 23: 329. 
Now: Oenanthe scotocerca scotocerca (von Heuglin, 1873)

MZUT Av8172 - dismounted skin; Eritrea, Keren (Bogos), August 1870; collected by 0. Antinori; from G. Doria in 1873.

Notes: Antinori et Salvadori (1874: 465) wrote "Philothamna fuscicaudata, Salvad. et Ant." and listed three specimens, including Av8172, but from the following text we clearly deduce that the authors did not want to attribute to themselves the description of a new species. Probably the confusion comes from the fact that in the same work, on p. 466, Antinori and Salvadori described the new genus Philothamna (sic).

Salvadori (1915: 28) correctly attributes fuscicaudata to Blanford and recalls that Phylothamna fuscicaudata is the type (-species) of the new genus Phylothamna Salvadori, but he forgets Antinori as a co-author!

According to van den Elzen et al. $(2011: 167,171)$ the description (the text) of Saxicola scotocerca Heuglin was published before 3 December 1869. Even if a plate of the species was issued only in 1873, we do not understand why Dickinson et Christidis (2014) postponed the description date from 1969 to 1873 . We also believe that Ruticilla fuscicaudata Blanford could be considered as a senior synonym, having been published on November 1, 1869 (Evenhuis 2003: 25).

\section{TURDIDAE}

Cochoa beccarii Salvadori, 1879

Salvadori T., 1879. Ann. Mus. Civ. Genova, vol. XIV: 228.

Now: Cochoa beccarii Salvadori, 1879

MZUT Av9568 - Indonesia, Sumatra, Singgalang Mt., no date; collected by O. Beccari; from G. Doria.

Notes: Elter (1986: 149) considers this specimen as a type, but it is an incorrect opinion. Both syntypes described by Salvadori (1879b: 228) are hosted in Genoa Museum (Arbocco et al. 1978: 213; E. Borgo, pers. comm.).

Turdus erythrorhynchus Rüppell MS.

Never described.

Now: Turdus abyssinicus abyssinicus J. F. Gmelin, 1789

MZUT Av601 - female; Ethiopia, Abyssinia, 1832; collected by Rüppell and donated in 1839 to the Turin museum.

Notes: referred to as "cotype", but already as junior synonym of T. abyssinicus, in Salvadori (1915: 10-11) and quoted as syntype in Elter (1986: 474). Von Heuglin (1871: 207) points out the presence in the Frankfurt Museum of some specimens labelled "Turdus erythrorhynchus Rüpp.", which he identifies as Turdus olivacinus Bonaparte ${ }^{2}$ (1850).

As far as we know, the species has never been described by Rüppell (Sherborn 1926a: 2212-2213, Steinbacher 1949, Frank D. Steinheimer, pers. comm. to G. Aimassi, 1 October 2015), who probably just donated specimens with a "Turdus erythrorhynchus" label to the Museums of Turin and Frankfurt.

Turdus ignobilis debilis Hellmayr, 1902

Hellmayr C.E. 1902. Journ. für Orn., 50: 44-69.

Now: Turdus ignobilis debilis Hellmayr, 1902

MZUT Av4451 - skin; male; Ecuador, Gualaquiza, November 1895; collected by E. Festa (field number 63).

Notes: in the MZUT collection are present three of the seven individuals captured by E. Festa in Ecuador and identified as Turdus ignobilis Sclater (Salvadori et Festa 1899a: 3). Salvadori (1915) does not mention them, but Elter (1986: 474) indicates the MZUT Av4451 specimen as syntype of Turdus ignobilis debilis.

2 In Seebohm (1881: 228) both T. erythrorhynchus Rüppell “fide Heugl." and T. olivacinus Bonaparte are treated as junior synonyms of T. abyssinicus Gmelin. 
Hellmayr (1902: 54), in the paper in which he describes Turdus ignobilis debilis, quotes (N. 3-4) a male and a female captured by E. Festa in Gualaquiza, seen by him in the Turin Museum, however (p. 56) he explicitly writes that he considers as types only two specimens captured by Natterer in Brazil, Rio Madeira, thus excluding from the type-series all the other specimens examined (ICZN 1999, Article 72.4.6).

\section{APPENDIX 2 - LOST TYPES}

Some types reported by Salvadori (1915) are no longer hosted in the MZUT Collection and already Elter (1986) had not listed them. Most likely some of these were destroyed in the course of a bombing during World War II. In this appendix we list alphabetically the corresponding binomina, according to the genera of the original descriptions.

Acridotheres leucocephalus Giglioli \& Salvadori, 1870

Atti R. Acc. Sc. Torino, Vol. 5: 273.

Now: Acridotheres burmannicus leucocephalus Giglioli \& Salvadori, 1870

The lost specimen was the holotype.

Ammomanes assabensis Salvadori, 1902

Boll. Mus. Zool. An. comp. Univ. Torino, No. 425 (vol. XVII), pp. 1-2.

Now: Ammomanes deserti assabensis Salvadori, 1902

Despite the statement Salvadori (1915: 40), in addition to the two syntypes of the Turin

Museum, now dispersed, the type series included a third specimen, at that time housed

in the Museum of the University of Rome.

Ocypterus superciliosus Gould, 1837

Synopsis Birds Australia, Part I, Plate 1, fig. 2.

Now: Artamus superciliosus (Gould, 1837)

Calornis enganensis Salvadori, 1892

Ann. Mus. Civ. St. Nat. Genova, Vol. XXXII, p. 137.

Now: Aplonis panayensis enganensis (Salvadori, 1892)

Five syntypes are housed in the Genoa Museum (Arbocco et al. 1979: 243).

Cisticola cinereola Salvadori, 1888

Ann. Mus. Civ. St. Nat. Genova, Vol. XXVI, p. 254.

Now: Cisticola cinereolus cinereolus Salvadori, 1888

The lost specimen was the holotype.

Gracula robusta Salvadori, 1887

Ann. Mus. Civ. St. Nat. Genova, Vol. XXIV, p. 554.

Now: Gracula religiosa robusta Salvadori, 1887

Five syntypes are housed in the Genoa Museum (Arbocco et al. 1979: 244).

Habropyga rara Antinori, 1864

Catalogo descrittivo di una collezione di uccelli [...], pp. 72-73.

Now: Lagonosticta rara rara (Antinori, 1864)

The lost specimen was the holotype.

Heterophasia simillima Salvadori, 1879

Ann. Mus. Civ. St. Nat. Genova, Vol. XIV, p. 232.

Now: Heterophasia picaoides simillima Salvadori, 1879

Three syntypes are housed in the Genoa Museum (Arbocco et al. 1979: 217).

Iyngipicus wattersi Salvadori and Giglioli, 1885

Atti R. Acc. Sc. Torino, Vol. 20, pp. 633-4.

Now: Dendrocopos canicapillus kaleensis (Swinhoe, 1863) 
Two specimens were described, however Salvadori (1915: 21) considered the Turin specimen as "the" type; Salvadori himself wrote that the second specimen was sent to the Count Turati of Milan.

Lagonosticta somaliensis Salvadori, 1894

Mem. R. Acc. Sc. Torino, serie 2a, Vol. 44, pp. 557.

Now: Lagonosticta senegala somaliensis Salvadori, 1894

The lost specimen was the holotype.

Lamprocolius defilippii Salvadori, 1866

Atti Soc. Ital. Sci. Nat. Milano, Vol. VIII [1865], pp. 379-380.

Now: doubtfully a synonym of Lamprotornis chrysonotis Swainson, 1837 (Shelley, 1906:

67)

According to Sharpe (1890: 171) and Salvadori (1915: 19) this species was never identified reliably. The loss of the holotype makes any future investigation unattainable.

Lamprotornis viridipectus Salvadori, 1894

Mem R. Acc. Sc. Torino, serie seconda, Vol. 44, pp. 560.

Now: Lamprotornis purpuroptera purpuroptera Rüppell, 1845

The type series included only two specimens housed in the Turin Museum, both missing.

Macronyx ascensi Salvadori, 1907

Boll. Mus. Zool. An. comp. Univ. Torino, No. 570 (vol. XXII), pp. 6-7.

Now: Macronyx fulleborni ascensi Salvadori, 1907

The lost specimen was the holotype.

Malacias castanopterus Salvadori, 1889

Ann. Mus. Civ. St. Nat. Genova, Vol. XXVII, pp. 363 and 411.

Now: Heterophasia melanoleuca castanoptera (Salvadori, 1889)

Two syntypes are housed in the Genoa Museum (Arbocco et al. 1979: 217).

Motacilla cinereocapilla Savi, 1831

Nuovo Giornale de' Letterati, N. 57, Tomo XXII, Parte Scientifica, pp. 186-193.

Now: Motacilla flava cinereocapilla Savi, 1831

Oriolus baruffii Bonaparte, 1850

Conspectus generum avium, [I], p. 347.

Now: Oriolus brachyrynchus brachyrynchus Swainson, 1837

Description based on specimens of Turin and Leiden Museums, but no types listed in Dekker (2003).

Oriolus moloxita "Buffon" Rüppell, 1836

Neue Wirbelthiere zu der Fauna von Abyssinien [...] Vögel, p. 29, pl. 12, fig. 1.

Now: Oriolus monacha monacha (J.F. Gmelin, 1789)

Oriolus rolleti Salvadori, 1864

Atti Soc. Ital. Sci. Nat. Milano, Vol. VII, pp. 160-161.

Now: Oriolus larvatus rolleti Salvadori, 1864

The type series included only two specimens housed in the Turin Museum, both missing.

Sericornis arfakiana Salvadori, 1876

Ann. Mus. Civ. St. Nat. Genova, Vol. VII, pp. 962-3.

Now: Sericornis nouhuysi cantans Mayr, 1930

According to Arbocco et al. (1979: 221) a lectotype and two paratypes (sic) are housed in the Genoa Museum, but we believe it is more appropriate to consider the three specimens as syntypes.

Sylvia sarda Temminck, 1820

Manuel d'Ornithologie, seconde édition, première partie, pp. 204-205.

Now: Curruca sarda (Temminck, 1820). 


\section{Acknowledgments}

In the many years we have dealt with this catalog, we have received the support of many people. We sincerely wish to thank all of them: Mark Adams, Maria Judite Alves, Giovanni Boano, Enrico Borgo, Murray Bruce, Pier Giuseppe Chiadò Fiorio, Giorgio Chiozzi, Paul Cooper, Edward C. Dickinson, Giuliano Doria, Neal Evenhuis, Fourcroy Florence, Sylke Frahnert, Ross Galbreath, Elena Gavetti, Christophe Gouraud, Daniele Grigion, Mary LeCroy, Carla Marangoni, Giuseppe Manganelli, Gerald Mayr, Ronald Mueller, Frank Rheindt, Maria Rosaria Scalas, Paul Scofield, Anna-Katharina Semmler, Marina Spini, Frank D. Steinheimer, Michael Stiller, Steven van der Mije, Carlo Violani, Claire Voisin.

Many thanks also to Jan Hušek, Jiřina Dašková (National Museum, Prague) and to an anonymous referee, who provided us with valuable contribution to improve our work.

\section{References}

Aimassi G., 2015: The original description of Bonelli's Eagle Aquila fasciata Vieillot (Aves: Accipitridae). - Zoological Bibliography 4, 1: 1-14.

Aimassi G. \& Dickinson E.C., 2018: Hemixus sumatranus Wardlaw Ramsay, 1882, and Hemixus sumatranus Salvadori, 1888. - Bulletin of the British Ornihologists' Club 138, 2: 135-139.

Aimassi G. \& Ghiraldi L., 2017: Lectotipi e Paralectotipi designati da Salvadori nella collezione ornitologica del Museo di Zoologia dell'Università di Torino. - In: Fasano S.G., Rubolini D. (a cura di), Riassunti del XIX Convegno Italiano di Ornitologia. Torino. Tichodroma 6: 68.

Allen J.A., 1889: On the Maximilian Types of South American Birds in the American Museum of Natural History. - Bulletin of the American Museum of Natural History, Vol. II: 209-276.

Antinori 0., 1856: Picus cruentatus, Antinori, n. sp. - Naumannia 6: 411-414.

Antinori 0., 1864: Catalogo descrittivo di una collezione di uccelli fatta da Orazio Antinori nell'interno dell'Affrica centrale nord dal maggio 1859 al luglio 1861. - Milano: G. Daelli e comp., $\mathrm{xxx}+117 \mathrm{pp}$.

Antinori 0. \& Salvadori T., 1874: Viaggio dei signori O. Antinori, G. Beccari ed A. Issel nel Mar Rosso, nel territorio dei Bogos e regioni circostanti durante gli anni 1870-71. - Annali del Museo civico di Storia naturale di Genova 4 [1873]: 366-520.

Arbocco G., Capocaccia L. \& Violani C., 1979: Catalogo dei Tipi di uccelli del Museo Civico di Storia Naturale di Genova. - Annali del Museo civico di Storia naturale di Genova 82: 184-265.

Arbocco G., Capocaccia L. \& Violani C., 1986: Catalogue of Bird Types in the collections of the Natural History Museum of Genoa: some addenda. - Annali del Museo civico di Storia naturale di Genova 86: 13-28.

Bahr N., 2011: The Bird Species. Systematics of the Bird Species and Subspecies of the World. Charadriiformes. - Minden, Germany: 191 pp.

Bangs O. \& Penard T.E., 1925: The Henry Bryant Types of Birds. - Bulletin of the Museum of Comparative Zoology 67, n. 3: 197-207.

Beccari O., 1902: Nelle foreste di Borneo. - Firenze: Tip. S. Landi, xvi + 667 pp.

Benvenuti E., 1863: Descrizione di quattro nuove specie della famiglia dei Trochilidi, provenienti dalla Nuova Granata e di una nuova specie di Sylvia del Brasile. - Firenze: Stamperia Reale, 16 pp.

Benvenuti E., 1866: Descrizione di quattro nuove specie della famiglia dei Trochilidi provenienti dalla Nuova Granata e di una nuova specie di Dendroica del Brasile, con l'aggiunta di una nota riguardante la fauna toscana. - Annali del R. Museo di Fisica e Storia naturale di Firenze, n.s., 1: 199-209. 
Bertoni A. de Winkelried, 1913: Contribución para un catálogo de aves argentinas. - Anales de la Sociedad Científica Argentina 75: 64-102.

Blanford W.T., 1869: Descriptions of five Birds and a Hare from Abyssinia. - Annals and Magazine of Natural History, ser. 4, 4, n. 23: 329-330.

Bocage J.V. Barboza du, 1878: Mélanges ornithologiques. IV. Espèces nouvelles d'Angola. Sciências, Mathemáticas, Physicas e Naturaes, Lisboa 23: 208-214.

Boitard M.P., 1824: Histoire naturelle des Oiseaux de proie d'Europe.- Paris: Parmantier Audot, 40 pp. + xv planches.

Bolau H., 1898: Die Typen der Vogelsammlung des Naturhistorischen Museums zu Hamburg. - Mitteilungen aus dem Naturhistorischen Museum in Hamburg 15 [1897]: 45-71.

Bonaparte C.L., 1831: Continuazione sulla seconda edizione del Regno Animale del Barone Cuvier. Osservazioni. - Annali di Storia naturale 4, fasc. XII: 303-389.

Bonaparte C.L., 1850: Conspectus Generum Avium. - Lugduni Batavorum: 543 pp.

Bonaparte C.L., 1856: Espèces nouvelles d'Oiseaux d'Asie et d'Amérique, et tableaux paralléliques des Pélagiens ou Gaviae. - Comptes rendus hebdomadaires des séances de l'Académie des Sciences, 42, n. 17: 764-776.

Bonaparte C.L., 1857: Conspectus Generum Avium. - Tom. II. Lugduni Batavorum: 232 pp.

Bonelli A., 1820: Note sur le Becfin de Cetti pag. 259. - Memorie della Reale Accademia delle Scienze di Torino 25: 261.

Bowen T., 2013: The type locality of Craveri's Murrelet Synthliboramphus craveri. - Marine Ornithology 41: 49-54.

Brizio D. (ed.), 1990: Giornale di Viaggio. Avventura ed esplorazione naturalistica in America Centrosettentrionale. 1855-1859. 2 Voll. - Bra, Italy: Museo civico Craveri.

Bruce M., 2011: “Gould, J. (1837-1838) A Synopsis of the Birds of Australia” p. 97. - In: Dickinson E.C., Overstreet L.K., Dowsett R.J. \& Bruce M.D.: Priority! The dating of Scientific Names in Ornithology. - Northampton, UK: Aves Press, 320 pp.

Bryant H., 1866: A List of Birds from Porto Rico. Proceedings of the Boston Society of Natural History 10 (1864-1866): 248-257.

Cooke W.W., 1916: The Type Locality of Brachyramphus craverii. - The Auk, n.s., 33: 80.

Cory C.B. \& Hellmayr C.E., 1927: Catalogue of Birds oft he Americas and the adjacent islands in Field Museum of Natural History. Part V. Tyrannidae. - Chicago: Field Museum of Natural History, Publication 242, 517 pp.

Cretzschmar P.J., 1829: Atlas zu der Reise im nördlichen Afrika von Eduard Rüppell, Part 14. - Frankfurt am Main: Brönner ed., Pag. 44-45, Plate 30.

Crockett, D.E., 1994: Rediscovery of Chatham Island taiko Pterodroma magentae. - Notornis (Supplement) 41: 49-60.

D’Albertis L.M. \& Salvadori T., 1879: Catalogo degli uccelli raccolti da L. M. D’Albertis durante la $2^{\mathrm{a}}$ e $3^{\mathrm{a}}$ esplorazione del Fiume Fly negli anni 1876 e 1877. - Annali del Museo civico di Storia naturale di Genova 14: 21-147.

De Filippi F., 1853: Fragment d'une lettre de M. Ph. Defilippi à son Altesse le Prince Bonaparte. - Révue et Magasin de Zoologie pure et appliquée, $2^{\mathrm{e}}$ sér., 5: 289-291.

De Filippi F., 1863: Nuove o poco note specie di Animali Vertebrati raccolte in un viaggio in Persia nell'estate dell'anno 1862. - Archivio per la Zoologia, l'Anatomia e la Fisiologia, Modena, 2, Fasc. 2: 378-393.

De Filippi F., 1865: Note di un viaggio in Persia nel 1862. - Milano, G. Daelli \& C. Editori, xi $+369 \mathrm{pp}$.

Degland C.D., 1849: Ornithologie européenne. Tome premier - Paris: 632 pp.

Dekker R.W.R.J., 2003. Type specimens of birds in the National Museum of Natural History, Leiden, Part. 2. - NNM Technical Bulletin 6: 1-142.

Dekker R.W.R.J. \& Quaisser C., 2006: Type specimens of birds in the National Museum of Natural History, Leiden. Part 3. Passerines: Pachycephalidae - Corvidae. NNM Technical Bulletin 9: 3-77. 
Dickinson E.C., 2011a: “Atti della R[eale]. Accademia delle Scienze di Torino”. Pp. 180-181 in: Dickinson E.C., Overstreet L.K., Dowsett R.J. \& Bruce M.D., Priority! The Dating of Scientific Names in Ornithology. - Northampton, UK: Aves Press.

Dickinson E.C., 2011b: "Memorie della Reale Accademia delle Scienze di Torino". Pp. 218-219 in: Dickinson E. C., Overstreet L.K., Dowsett R.J. \& Bruce M.D., Priority! The Dating of Scientific Names in Ornithology. Northampton, UK: Aves Press.

Dickinson E.C. \& Christidis L. (eds.), 2014: The Howard \& Moore Complete Checklist of the Birds of the World. 4th. Edition, Vol 2. - Eastbourne, UK: Aves Press, 752 pp.

Dickinson E.C., Dekker R.W.R.J., Eck S. \& Somadikarta S., 2002: Systematic notes on Asian birds. 23. Types of the Campephagidae. Zoologische Verhandlingen Leiden 340: 31-74.

Dickinson E.C. \& Remsen Jr. J.V. (eds.), 2013: The Howard \& Moore Complete Checklist of the Birds of the World. 4th. Edition, Vol 1. - Eastbourne, UK: Aves Press, 461 pp.

Doria G., 1878: I naturalisti Italiani alla Nuova Guinea. - Bollettino della Società Geografica Italiana. Anno XII, Vol. XV (Serie II, Vol. III): 154-169.

Duncan F.M., 1937: On the dates of publication of the Society's 'Proceedings', 1859-1926. Proceedings of the Zoological Society of London, A107: 71-84.

Elter O., 1986: La Collezione Ornitologica del Museo di Zoologia dell'Università di Torino. Museo Regionale di Scienze Naturali Torino, Cataloghi 8, 516 pp.

Evenhuis N.L., 2003: Publication and dating of the journals forming the Annals and Magazine of Natural History and the Journal of Natural History. - Zootaxa 385: 1-68.

Eyton T.C., 1838: Monograph of the Anatidae. - London: Longman, 183 pp.

Festa E., 1894: Viaggio del Dr. E. Festa in Palestina, nel Libano e regioni vicine. IX. Uccelli. Bollettino dei Musei di Zoologia ed Anatomia comparata della R. Università di Torino 174 (vol. IX): 1-7.

Festa E., 1925: Missione zoologica del Dr. E. Festa in Cirenaica. Uccelli (II). Bollettino dei Musei di Zoologia ed Anatomia comparata della R. Università di Torino, n.s., 24 (vol. XXXIX): 1-28.

Finsch 0. \& Hartlaub G., 1870: Baron Carl Claus von der Decken's Reisen in Ost-Afrika in den Jahren 1859-1865. Vierter Band: Die Vögel Ost-Afrikas. - Leipzig und Heidelberg: 897 pp. $+10 \mathrm{Pl}$.

Forbes W.A., 1879: A Synopsis of tlie Meliphagine Genus Myzomela, with descriptions of two new species. - Proceedings of the Zoological Society of London [1879]: 256-278.

Gadow H., 1883: Catalogue of the Passeriformes, or perching birds, in the collection of the British Museum. Ciclomorphae and Certhiomorphae. - London: the Trustees, 385 pp. + IX pl.

Galbreath R. \& Aimassi G., 2020: Atti della Società italiana di scienze naturali 1859-1895 and the dates of its parts. - Zoological Bibliography 6, 12: 135-150.

Gené G., 1834: Description de quelques espèces de la collection zoologique de Turin, indiquées par le Prof. Bonelli comme inédites ou mal connues. Memorie della R. Accademia delle Scienze di Torino 37: 291-298.

Gené G. [in Anonymous], 1839: Nouvelle espèce européenne du genre Faucon. Revue Zoologique, Tome II (Avril 1839): 105.

Gené G., 1840: Descrizione di un nuovo falcone di Sardegna (Falco eleonoræ). Memorie della Reale Accademia delle Scienze di Torino, Serie seconda, 2: 41-47.

Ghiraldi L. \& G. Aimassi, 2019: Extinct and Endangered ('E\&E') birds in the ornithological collection of the Museum of Zoology of Torino University, Italy. Bulletin of the British Ornihologists' Club 139, 1: 28-45.

Giglioli E.H., 1870: Note intorno alla distribuzione della fauna vertebrata nell'oceano, prese durante un viaggio intorno al globo 1865-68. - Firenze: Civelli, 96 pp.

Giglioli E.H., 1876: Viaggio intorno al Globo della r. pirocorvetta italiana Magenta negli anni 1865-1868. - Milano: Maisner e C., 1033 pp. 
Giglioli E.H., 1888: Note intorno agli animali vertebrati raccolti dal conte Augusto Boutourline e dal D.r Leopoldo Traversi ad Assab e nello Scioa negli anni 1884-87. - Annali del Museo civico di Storia naturale di Genova 2, 6: 5-73.

Giglioli E.H. \& Salvadori T., 1868: Nuove specie di Procellari raccolte durante il viaggio fatto intorno al mondo negli anni 1865, 1866, 1867, 1868 dalla pirocorvetta italiana Magenta. Estratto dagli Atti della Società italiana di scienze naturali 11, Fasc. 3. - Milano: Bernardoni, 9 pp.

Giglioli E.H. \& Salvadori T., 1869a: Nuove Specie di Procellari raccolte durante il viaggio fatto intorno al mondo negli anni 1865, 1866, 1867, 1868 dalla pirocorvetta italiana Magenta. - Atti della Società Italiana di Scienze Naturali 11: 450-458.

Giglioli E.H. \& Salvadori T., 1869b: On some new Procellariidae collected during a Voyage round the World in 1865-68 by H.I.M.'s S. 'Magenta'. - Ibis, n.s., 5: 61-68.

Giglioli E.H. \& Salvadori T., 1870a: Altre nuove o poco note specie di Uccelli raccolte durante il viaggio fatto intorno al mondo dalla pirocorvetta italiana Magenta negli anni 1865, 1866, 1867, 1868. - Atti della Reale Accademia delle Scienze di Torino 5: 273-276.

Giglioli E.H. \& Salvadori T., 1870b: On some other new and little-known Birds, collected during the Voyage round the World in 1863-68 of H. I. M.'s S. Magenta. - Ibis, n.s., 6 (n. XXII - April): 185-187.

Gmelin J.F., 1789: Caroli a Linné Systema Naturae, per regna tria Naturae: secundum Classes, Ordines, Genera, Species, cum Characteribus, Differentiis, Synonymis, Locis. Part II. Lipsiae (Leipzig, Germany): Impensis G. E. Beer, pp. 501-1032.

Gould J., 1837: A Synopsis of the Birds of Australia and the adjacent islands. Part I-II. London: The Autor.

Gould J., 1838a: Exhibition of Australian Birds from his own Collection, and characters of New Species [Mr. Gould exhibited from his Australian collection two species of the genus Platycercus...]. - Proceedings of the Zoological Society of London, Part V [1837]: 88-89.

Gould J., 1838b: A Synopsis of the Birds of Australia, and the adjacent islands. Part III-IVAppendix. - London: The author.

Gould J., 1843. Descriptions of thirty New Species of Birds from Australia [Mr. Gould exhibited and characterized the following thirty new species of Australian Birds]. - Proceedings of the Zoological Society of London, Part X [1842]: 131-142.

Gould J., 1863: Descriptions of Sixteen New Species of Birds from the Island of Formosa, collected by Robert Swinhoes, Esq., Her Majesty's Vice-Consul at Formosa. - Proceedings of the Zoological Society of London [1862]: 280-286.

Gouraud C., 2015: List of type specimens of birds in the Baillon Collection (La Châtre, France). Part. 1. Non-Passerines. - Bulletin of the British Ornithologists' Club 135, 2: 131-153.

Grant C.H.B. \& Mackworth-Praed C.W., 1936: On the correct type-locality of Hemiparra crassirostris crassirostris. - Bulletin of the British Ornithologists' Club 56, n. 393: 92-93.

Gray G.R., 1867: List of the Specimens of Birds in the Collection of the British Museum. Part V. Gallinae. - London: The Trustees, $120 \mathrm{pp}$.

Greenway J.C., jr., 1973: Type specimens in the American Museum of Natural History. Part 1. - Bulletin of the American Museum of Natural History 150: 207-346.

Greenway J.C., 1978: Type Specimens of Birds in the American Museum of Natural History. Part 2. - Bulletin of the American Museum of Natural History 161: 1-306.

Griscom L., 1932: Notes on imaginary species of Ramphocelus. - Auk 49: 199-203.

Hartert E., 1910: Die Vögel der paläarktischen Fauna. Band I. - Berlin: Friedländer und Sohn, $832 \mathrm{pp}$.

Hartlaub G., 1855: Ueber Chettusia crassirostris de Filippi. - Journal für Ornithologie 5, 5: 427.

Hartlaub G., 1868: On a Collection of Birds from some less-known Localities in the Western Pacific. - Proceedings of the Zoological Society of London [1867]: 828-832. 
Hartlaub G. \& Finsch 0., 1872: On a fourth Collection of Birds from the Pelew and Mackenzie Islands. - Proceedings of the Zoological Society of London [1872]: 87-114.

Hartlaub G. \& Monteiro J.J., 1860: On some Birds collected in Angola. - Proceedings of the Zoological Society of London 28: 109-112.

Hartmann R., 1866: Orazio Antinori: Beschreibung ind Verzeichniss einer von Mai 1859 bis Juli 1861 in Nord-Central-Afrika angelegten Vögelsammlung. - Journal für Ornithologie 14, 3: 191-208.

Hellmayr C.E., 1902: Revision einiger neotropischen Turdidae. - Journal für Ornithologie 50, 1: 44-69.

Hellmayr C.E. \& Conover B., 1942: Catalogue of Birds of the Americas and the adjacent islands in Field Museum of Natural History. Part I, Number 1. - Field Museum of Natural History, Publication 514, Zoological Series, Vol. XIII, 636 pp.

Hellmayr C.E. \& Conover B., 1948: Catalogue of Birds of the Americas and the adjacent islands in Field Museum of Natural History. Part I, Number 3. - Field Museum of Natural History, Publication 616, Zoological Series, Vol. XIII, 383 pp.

Heuglin M.T. von, 1867: Berichtigungen und Noten zu Antinori's Katalog der nordostafrikanischen Vögel. - Journal für Ornithologie 15, 3: 198-207.

Heuglin M.T. von, 1868: Note on Hypocolius ampelinus, Bp. - Ibis, n.s., 4, n. 13: 181-183, Pl. 5.

Heuglin M.T. von, 1869: The Malurinae of North-eastern Africa. - Ibis, n.s., 4: 79-107.

Heuglin M.T. von, 1869b: Ornithologie Nordost-Afrika's. Erster Band. - Cassel: V. \& T- Fischer, 108+416 pp. + 16 Taf.

Heuglin M.T. von, 1871: Ueber die rothäugige Drossel, Turdus olivacinus Bp. - Journal für Ornithologie 19, 3: 206-208.

Holub E. \& Pelzeln A. von, 1882: Beiträge zur Ornithologie Südafrikas. - Wien: Alfred Hölder und Universitäts-Buchhändler, 385 pp.

Horsfield T., 1821. Systematic Arrangement and Description of Birds from the Island of Java. - Transactions of the Linnean Society of London 13: 133-200.

del Hoyo J., Elliott A. \& Sartagal J. (eds.), 1996: Handbook of the Birds of the World. Vol. 3. Barcelona: Lynx Edicions, $821 \mathrm{pp}$.

del Hoyo J. \& Collar N.J., 2014: HBW and BirdLife International Illustrated Checklist of the Birds of the World. Volume 1: Non-passerines. - Barcelona: Lynx Edicions, 903 pp.

del Hoyo J. \& Collar N.J., 2016: HBW and BirdLife International illustrated checklist of the Birds of the World, Volume 2: passerines. - Barcelona: Lynx Edicions, 1013 pp.

Imber M.J., Tennyson A.J.D, Taylor G.A. \& Johnston P., 1998: A second intact specimen of the Chatham Island Taiko (Pterodroma magentae). - Notornis 45, 4: 247-254.

ICZN, 1985: International code of zoological nomenclature. Third ed. - London: The International Trust for Zoological Nomenclature, $338 \mathrm{pp}$.

ICZN, 1999: International code of zoological nomenclature. Fourth ed. - London: The International Trust for Zoological Nomenclature, $132 \mathrm{pp}$.

ICZN, 2012: Amendment of Articles 8, 9, 10, 21 and 78 of the International Code of Zoological Nomenclature to expand and refine methods of publication. - Bulletin of Zoological Nomenclature 69, 3: 161-169.

Jerdon T.C., 1839: Catalogue of the Birds of the Peninsula of India. II. - Madras Journal of Literature and Science 25: 234-269.

Jerdon T.C., 1864: The birds of India, Vol. III (with appendix). - Calcutta: G. Wyman \& Co., $876+$ xxxii pp.

Kennerley P.R., Bakewell D.N. \& Round P.D., 2008: Rediscovery of a long-lost Charadrius plover from South-East Asia. - Forktail 24: 63-79.

LeCroy M., 2012: Type specimens of birds in the American Museum of Natural History. Part 10. - Bulletin of the American Museum of Natural History. Number 368, 125 pp.

Lesson R.P., 1830: Traité d'Ornithologie, Livr. No. 4 (pp. 241-320). - Paris, F. G. Levrault. 
Levaillant F., 1800 [1799 on frontispice]: Oiseaux d'Afrique, Tome second. - Paris : chez J.J. Fuchs, 206 pp., Plates 50-97.

Levaillant F., 1807: Histoire Naturelle des Couroucous et des Touracos (troisième partie) et Supplément aux differents genres d'Oiseaux décrits dans les deux premiers volumes. Paris: Chez Denné le Jeune, 52 pp.

Lichtenstein M.H.C., 1818: Verzeichniss von ausgestopften Saeugethieren und Voegeln [...]. - Berlin, 39 pp.

Lichtenstein M.H.C., 1854: Nomenclator Avium Musei Zoologici Berolinensis. - Berlin: Buchdruckerei der Königlichen Akademie der Wissenschaften, 123 pp.

Maretti S., Razzetti E. \& Chiozzi G. (eds.), 2013: L'esplorazione zoologica in Africa. Le ricerche degli italiani. - Natura 103, Fasc. I, 232 pp.

Marmora A. della, 1820: Mémoire sur deux oisseaux (sic) du Comté de Nice, observés en novembre et décembre 1819. - Memorie della Reale Accademia delle Scienze di Torino 25: $253-260$.

Marmora A. della, 1834: Détermination et description des différences d'âge de l'Aigle Bonelli (Falco Bonelli, Temm.). - Memorie della Reale Accademia delle Scienze di Torino 37: 110-125.

Mathews G.M. \& Iredale T., 1920: Sherborn and the Systematist. - The Austral avian record 4, 4\&5: 131-132.

Matschie P. \& Reichenow A., 1892: Bericht über die Februar-Sitzung (Reichenow: Neue afrikanische Vogelarten). - Journal für Ornithologie 40, II Heft (No. 198): 218-222.

Mayr E., 1941: List of New Guinea Birds. - New York: The American Museum of Natural History, $260 \mathrm{pp}$.

Mayr E. \& Cottrell G.W. (eds.), 1979: Check-list of Birds of the World. Volume I. Second edition. Revision of the Work of James L. Peters. - Cambridge: Museum of Comparative Zoology, $547 \mathrm{pp}$.

Mayr E. \& Cottrell G.W. (eds.), 1986: Check-list of Birds of the World. Volume XI. A Continuation of the Work of James L. Peters. - Cambridge: Museum of Comparative Zoology, 638 pp.

Mayr E. \& Greenway J.C. jr. (eds.), 1960: Check-list of Birds of the World. Volume IX. A Continuation of the Work of James L. Peters. Cambridge, Cambridge: Museum of Comparative Zoology, 506 pp.

Mayr E. \& Greenway C. (eds.), 1962: Check-list of Birds of the World. Volume XV. A Continuation of the Work of James L. Peters. - Cambridge: Museum of Comparative Zoology, 315 pp.

Mazzotti S., 2011: Esploratori perduti. - Torino: Codice edizioni, 239 pp.

Mearns B. \& Mearns R., 1998: The Bird Collectors. - San Diego, London: Academic Press, $472 \mathrm{pp}$.

Meyer A.B., 1874: Über neue und ungenügend bekannte Vögel von Neu-Guinea und den Inseln der Geelvinksbai (Erste Mittheilung). - Sitzungsberichte der Mathematisch Naturwissenschaftlichen Classe der Kaiserlichen Akademie der Wissenschaften 69, Heft I-II: 74-90.

Meyer A.B. \& Wiglesworth L.W., 1894: Beschreibung einiger neuen Vögel der Celébes-Region. - Journal für Ornithologie 42 (2 - april): 113-116.

Michahelles K., 1830: Ueber einige Dalmatinische Vertebraten, die zugleich im westlichen Asien vorkommen. - Isis von Oken 23 (8): col. 809-820.

Nehrkorn A., 1885: Zur Avifauna der Insel Waigen. - Journal für Ornithologie 33, Heft I (n. 169, Januar): 30-35.

Neumann 0., 1903a: New subspecies of African birds. - Bulletin of the British Ornithologists' Club 14: 15-16.

Neumann 0., 1903b: Über einige afrikanische Vögel. I, Über Malaconotus. - Ornithologische Monatsberichte 11, 9: 87-90.

Neumann O., 1905: Vögel von Schoa und Süd-Āthiopien. - Journal für Ornithologie 53, Heft I: $184-243$. 
Oberholser H.C., 1899: A Synopsis of the Genus Contopus and Its Allies. - The Auk 16, 4: 330-337.

Olson S.L. \& Violani C., 1995: Some unusual hybrids of Ramphocelus, with Notes on evolution in the Genus (Aves: Thraupinae). - Bollettino del Museo Regionale di Scienze Naturali Torino 13, 1: 297-312.

Passerin d'Entrèves P., Rolando A. \& Violani C., 1986: Tommaso Salvadori nel centocinquantenario della nascita (1835-1923). - Rivista italiana di Ornitologia 56, 3-4: 133-171.

Passerin d'Entrèves P. \& Sella Gentile G., 1985: Franco Andrea Bonelli Zoologo trasformista. - Studi Piemontesi 14, 1: 33-48.

Passerin d'Entrèves P., Violani C. \& Rolando A., 1995: The Himalayan bird collection of the Marquis Paolo Solaroli, "Prince of Sirdanah", and the type locality of Oedicnemus indicus Salvadori, 1865. - Bulletin of the British Ornithologists' Club 115, 2: 128-129.

Paynter R.A. jr. (ed.), 1967: Check-list of Birds of the World. Volume XII. A Continuation of the Work of James L. Peters. - Cambridge: Museum of Comparative Zoology, 495 pp.

Pelzeln A., von, 1865: Vögel. - In: von Wüllerstorf-Urbair B., Reise der Österreichischen Fregatte Novara um die erde in den Jahren 1857, 1858, 1859. Zoologischer Teil. Erster Band. - Wien: Aus der Kaiserlich-Königlichen Hof- und Staatsdruckerei, 176 pp. + VI Taf.

Peters J.L., 1923: El nombre de la "Paloma de monte". - Hornero 3, 02: 200.

Peters J.L., 1931: Check-list of Birds of the World. Volume I. - Cambridge, H.U.P., 345 pp.

Peters J.L., 1934: Check-list of Birds of the World. Volume II. - Cambridge, H.U.P., 401 pp.

Peters J.L., 1937: Check-list of Birds of the World. Volume III. - Cambridge, H.U.P., 311 pp.

Peters J.L., 1940: Check-list of Birds of the World. Volume IV. - Cambridge, H.U.P., 291 pp.

Pierantoni U., 1925: Tommaso Salvadori. - Bollettino dei Musei di Zoologia ed Anatomia comparata della R. Università di Torino, n.s., n. 39 (vol. XV): 1-24.

Poggi R., 1996: Use of archives for nomenclatorial purposes: clarifications and corrections of the dates of issue for volumes 1-8 (1870-1876) of the Annali del Museo civico di Storia naturale di Genova. - Archives of Natural History 23, 1: 99-105.

Poggi R., 2010: Gli «Annali» pubblicati dal Museo Civico di Storia Naturale "Giacomo Doria" di Genova: storia del periodico ed indici generali dei primi cento volumi (1870-2009). Annali del Museo Civico di Storia Naturale “Giacomo Doria” 101: 1-529.

Reichenow A., 1892: Zur Vogelfauna von Kamerun. Erster Nachtrag. - Journal für Ornithologie 40, II Heft (No. 198): 177-195.

Reichenow A., 1900: Die Vögel Afrikas. Erster Band. - Neudamm: J. Neumann Verlag, 706 pp.

Roselaar C.S., 2003: An inventory of major European bird collections. Bulletin of the British Ornithologists' Club 123A: 253-337.

Rüppell E., 1835-1840: Neue Wirbelthiere zu der Fauna von Abyssinien gehörig. Vögel. Frankfurt am Main: 116 pp., 42 Taf.

Rüppell E., 1845: Systematische Uebersicht der Vogel Nord-Ost-Afrika's. - Frankfurt A.M.: $140 \mathrm{pp}+50$ Taf.

Salvadori T., 1864: Intorno ad alcune specie nuove o poco conosciute d'uccelli del Museo di Torino. - Atti della Società Italiana di Scienze Naturali 7: 149-162.

Salvadori T., 1865: Reale Accademia delle Scienze di Torino. Adunanza della Classe di scienze fisiche e matematiche. Tenuta il giorno 7 maggio 1865: Descrizione di una nuova Specie d'Avvoltoio. - Gazzetta Ufficiale del Regno d'Italia 126.

Salvadori T., 1865b: Descrizione di una nuova specie d'Avoltojo (gen. Gyps, Savig.). - Rivista delle Alpi, degli Appennini e Vulcani, Anno II: 167 - 173.

Salvadori T., 1866a: Descrizione di altre nuove Specie di Uccelli esistenti nel Museo di Torino. - Atti della Società Italiana di Scienze Naturali 8 [1865]: 375-389.

Salvadori T., 1866b: Intorno a due nuovi generi di uccelli. - Atti della Società Italiana di Scienze Naturali 8 [1865]: 371-374. 
Salvadori T., 1868a: Due nuove specie di uccelli della famiglia dei Caprimulgidi. - Estratto dagli Atti della Società italiana di scienze naturali 11, Fasc. III. Milano: Bernardoni, 4 pp.

Salvadori T., 1868b: Studio intorno ai lavori ornitologici del Professore Filippo De Filippi. Atti della Reale Accademia delle Scienze di Torino 3: 257-296.

Salvadori T., 1868c: Nota intorno al genere Prionochilus Strickl. - Atti della Reale Accademia delle Scienze di Torino 3: 412-420.

Salvadori T., 1868d: Nuove specie di Uccelli di Borneo. - Atti della Reale Accademia delle Scienze di Torino 3: 524-533.

Salvadori T., 1869a: Descrizione di una nuova Specie d’Avvoltoio. Pp. 129-136 In: Sobrero Ascanio. Notizia storica dei lavori fatti dalla classe di scienze fisiche e matematiche della Reale Accademia di scienze di Torino negli anni 1864 e 1865. - Torino: stamp. Reale.

Salvadori T., 1869b: Nota intorno ad alcuni uccelli di Costa Rica. - Atti della Reale Accademia delle Scienze di Torino 4: 170-185.

Salvadori T., 1869c: Monografia del Genere Ceyx Lacépède. - Atti della Reale Accademia delle Scienze di Torino 4: 440-476.

Salvadori T., 1869d: Due nuove specie di uccelli della famiglia dei Caprimulgidi. - Atti della Società Italiana di Scienze Naturali 11, fasc. 3: 447-450.

Salvadori T., 1870a: Nuove specie di uccelli dei generi Saxicola, Brachypus, Homochlamys Nov. Gen. - Atti della Reale Accademia delle Scienze di Torino 5 [1869]: 507-512

Salvadori T., 1871: Nuove specie di uccelli dei generi Criniger, Picus ed Homoptila Nov. Gen. - Atti della Reale Accademia delle Scienze di Torino 6: 128-132.

Salvadori T., 1872: Fauna d'Italia. Parte seconda: Uccelli. - Milan: lvi+352 pp.

Salvadori T., 1874a: Catalogo Sistematico degli Uccelli di Borneo con note ed osservazioni di G. Doria ed O. Beccari intorno alle specie da essi raccolte nel Ragiato di Sarawak. - Annali del Museo civico di Storia naturale di Genova 5, lii + 431 pp.

Salvadori T., 1874b: Nuove Specie di Uccelli delle Isole Aru e Kei raccolte da Odoardo Beccari e descritte da Tommaso Salvadori. - Annali del Museo civico di Storia naturale di Genova 6: 73-80. [IX-XI.1874 Poggi 2010: 343]

Salvadori T., 1874c: Altre nuove specie di Uccelli della Nuova Guinea e di Goram raccolte dal Signor L.M. D’Albertis e descritte da Tommaso Salvadori. - Annali del Museo civico di Storia naturale di Genova 6: 81-88.

Salvadori T., 1874d: Intorno al Genere Cymborhynchus Vigors. - Atti della Reale Accademia delle Scienze di Torino 9: 418-429.

Salvadori T., 1874e: Altre nuove specie di Uccelli raccolte nella Nuova Guinea dal Signor L.M. D’Albertis e nelle Isole Aru e Kei dal Dott. O. Beccari descritte da T. Salvadori. - Annali del Museo civico di Storia naturale di Genova 6: 308-314.

Salvadori T., 1874f (1875?): Intorno al genere Hermotimia Rchb. - Atti della Reale Accademia delle Scienze di Torino 10: 201-234.

Salvadori T., 1875a: Descrizione dell'Harpyopsis novae guineae, nuovo genere e nuova specie di rapace della sottofamiglia degli Accipitrini, raccolta dal Sig. L.M. D’Albertis nella Nuova Guinea. - Annali del Museo civico di Storia naturale di Genova 7: 682-683.

Salvadori T., 1875b: Catalogo di una collezione di uccelli del gruppo di Halmahera e di varie località della Papuasia, inviati in dono al Museo Civico di Genova dal Sig. A.A. Bruijn. Annali del Museo civico di Storia naturale di Genova 7: 749-796.

Salvadori T., 1876a: Descrizione di cinquantotto nuove specie di uccelli, ed osservazioni intorno ad altre poco note, della Nuova Guinea e di altre Isole Papuane, raccolte dal Dr. Odoardo Beccari e dai cacciatori del sig. A.A. Bruijn. - Annali del Museo civico di Storia naturale di Genova 7 [1875]: 896-976.

Salvadori T., 1876b: Descrizione di sei nuove specie di Uccelli delle Molucche, delle Kei e delle Aru e del maschio della Pachycephala lineolata, Wall. - Annali del Museo civico di Storia naturale di Genova 7 [1875]: 983-990. 
Salvadori T., 1876c: Catalogo di una seconda collezione di uccelli raccolti dal Sig. L.M. D’Albertis nell'Isola Yule e sulla vicina costa della Nuova Guinea, e di una piccola collezione della regione bagnata dal Fiume Fly. - Annali del Museo civico di Storia naturale di Genova 9: 7-49.

Salvadori T., 1876d: Intorno a due piccole collezioni di Uccelli, l'una di Pettà (Isole Sanghir) e l'altra di Tifore e di Batang Keteil, inviate dal Signor A. A. Bruijn al Museo Civico di Genova. - Annali del Museo civico di Storia naturale di Genova 9: 50-65.

Salvadori T., 1877: Intorno alle specie del genere Talegallus. - Annali del Museo civico di Storia naturale di Genova 9 (26 March 1877): 327-334.

Salvadori T., 1877a: Catalogo della prima collezione di uccelli fatta nella Nuova Guinea nel 1872 dal Sig. L.M. D’Albertis. - Annali del Museo civico di Storia naturale di Genova 10: 111-167.

Salvadori T., 1877b: Prodromus Ornithologiae Papuasiae er Moluccarum. III. Psittaci. - Annali del Museo civico di Storia naturale di Genova 10: 21-37.

Salvadori T., 1877c: Prodromus Ornithologiae Papuasiae et Moluccarum. IV. Bucerotidae, Meropidae, Alcedinidae, Coraciidae, Podargidae, Caprimulgidae, Cypselidae. - Annali del Museo civico di Storia naturale di Genova 10: 299-312.

Salvadori T., 1878a: Descrizione di una nuova specie di uccello del Genere Chalcopsittachus, Bp. e note intorno ad altre specie di uccelli nella Nuova Guinea. - Atti della Reale Accademia delle Scienze di Torino 13: 309-316.

Salvadori T., 1878b: Catalogo di una Collezione di uccelli di Tarawai fatta dai cacciatori del Sig. A.A. Bruijn. - Atti della Reale Accademia delle Scienze di Torino 13: 317-324.

Salvadori T., 1878c: Descrizione di una nuova specie del genere Lanius. - Annali del Museo civico di Storia naturale di Genova 12: 316.

Salvadori T., 1878d: Descrizione di trentuna specie nuove di uccelli della sottoregione papuana, e note intorno ad altre poco conosciute. - Annali del Museo civico di Storia naturale di Genova 12: $317-347$.

Salvadori T., 1878e: Osservazioni intorno alla supposta identità specifica della Rectes cirrhocephala (Less.) e della Rectes dichroa, Bp., e descrizione di due nuove specie del genere Rectes, Rchb. - Annali del Museo civico di Storia naturale di Genova 12: 471-474.

Salvadori T., 1879a: On Melaniparus semilarvatus. - Ibis, $4^{\text {th }}$ ser., vol. 3, n. 11: 300-303, Pl. IX..

Salvadori T., 1879b: Catalogo di una collezione di uccelli fatta nella parte occidentale di Sumatra dal Prof. Odoardo Beccari e descritta da Tommaso Salvadori. - Annali del Museo civico di Storia naturale di Genova 14: 169-253.

Salvadori T., 1880a: Prodromus Ornithologiae Papuasiae et Moluccarum. IX. Menuridae, Certhiidae, Nectariniidae, Dicaeidae, Meliphagidae. - Annali del Museo civico di Storia naturale di Genova 16: 62-82.

Salvadori T., 1880b: Ornitologia della Papuasia e delle Molucche. Parte Prima. - Torino: Paravia, $575 \mathrm{pp}$.

Salvadori T., 1880c: Osservazioni intorno ad alcune specie del Genere Collocalia, G.R. Gr. - Atti della Reale Accademia delle Scienze di Torino 15: 343-350.

Salvadori T., 1881a: Della vita e delle opere dell'ornitologo inglese John Gould. - Atti della Reale Accademia delle Scienze di Torino 16: 789-810.

Salvadori T., 1881b: Ornitologia della Papuasia e delle Molucche. Parte seconda. - Torino: Paravia, xvi $+707 \mathrm{pp}$.

Salvadori T., 1882a: Ornitologia della Papuasia e delle Molucche. Parte terza. - Torino: Paravia, $\mathrm{xv}+597 \mathrm{pp}$.

Salvadori T., 1882b: Descrizione di una nuova specie del Genere Collocalia ed osservazioni intorno alla C. infuscata, Salvad. - Atti della Reale Accademia delle Scienze di Torino 17: $304-306$.

Salvadori T., 1884: Spedizione Italiana nell'Africa equatoriale. Risultati Zoologici. Uccelli dello Scioa e della regione fra Zeila e lo Scioa. - Annali del Museo civico di Storia naturale di Genova 21: 7-276. 
Salvadori T., 1886: On some Papuan, Moluccan, and Sulu Birds. - Ibis, ser. 5a, vol. IV, n. XIV (april): 151-155.

Salvadori T., 1887a: Elenco degli uccelli italiani. - Annali del Museo civico di Storia naturale di Genova 23: 5-331.

Salvadori T., 1887b: Catalogo delle collezioni ornitologiche fatte presso Siboga in Sumatra, e nell'isola Nias dal Signor Elio Modigliani. - Annali del Museo civico di Storia naturale di Genova 24: 514-563.

Salvadori T., 1888a: Uccelli dello Scioa e dell'Harar raccolti dal Dott. Vincenzo Ragazzi. - Annali del Museo civico di Storia naturale di Genova 26: 525-544.

Salvadori T., 1888b: Catalogo di una Collezione di Uccelli dello Scioa fatta dal Dott. Vincenzo Ragazzi negli anni 1884, 1885, 1886. - Annali del Museo civico di Storia naturale di Genova 26: $185-326$.

Salvadori T., 1888c: Descrizione di una nuova specie del genere Hemixus raccolta in Sumatra dal Dott. O. Beccari. - Annali del Museo civico di Storia naturale di Genova 25: 525-527.

Salvadori T., 1888d: Viaggio di Leonardo Fea nella Birmania e nelle regioni vicine. III. Uccelli raccolti nel Tenasserim (1887). - Annali del Museo civico di Storia naturale di Genova 25: 554-622.

Salvadori T., 1889a: Descrizione di tre nuove specie di uccelli raccolte nei Monti Carin da Leonardo Fea. - Annali del Museo civico di Storia naturale di Genova 27: 363-364.

Salvadori T., 1889b: Viaggio di Leonardo Fea nella Birmania e nelle regioni vicine - XIX. Uccelli raccolti nei Monti Carin a Nord-Est di Tounghoo nel Pegù presso Rangoon e Tounghoo e nel Tenasserim presso Malewoon. - Annali del Museo civico di Storia naturale di Genova 27: 369-438.

Salvadori T., 1889c: Aggiunte alla Ornitologia della Papuasia e delle Molucche. Parte I. Accipitres - Psittaci - Picariae. - Torino: C. Clausen, pp. 1-64.

Salvadori T., 1890a: Aggiunte alla Ornitologia della Papuasia e delle Molucche. Parte II. Passeres. - Torino: C. Clausen, pp. 65-167.

Salvadori T., 1890b: Aggiunte alla Ornitologia della Papuasia e delle Molucche. Parte II. Passeres. - Memorie della R. Accademia delle Scienze di Torino, serie 2a, vol. 40: 193-293.

Salvadori T., 1891: Catalogo di una collezione di uccelli di Sumatra fatta dal Dott. Elio Modigliani. - Annali del Museo civico di Storia naturale di Genova 32: 41-78.

Salvadori T., 1892: Uccelli di Engano raccolti dal Dott. E. Modigliani. - Annali del Museo civico di Storia naturale di Genova 32: 123-142.

Salvadori T., 1894a: Intorno alla Pyrrhura chiripepé (Vieill.) e descrizione di una nuova specie del genere Pyrrhura. - Bollettino dei Musei di Zoologia ed Anatomia comparata della R. Università di Torino, No. 190 (vol. IX): 1-4.

Salvadori T., 1894b: Uccelli del Somali raccolti da D. Eugenio dei Principi Ruspoli. - Memorie della R. Accademia delle Scienze di Torino, serie 2a , vol. 44: 547-564.

Salvadori T., 1894c: Viaggio del Dr. Elio Modigliani nelle Isole Mentawei - Catalogo di una Collezione di Uccelli di Si-Pora. - Annali del Museo civico di Storia naturale di Genova 34: 588-601.

Salvadori T., 1895: Viaggio del dott. Alfredo Borelli nella Repubblica Argentina e nel Paraguay. XVI Tommaso Salvadori. Uccelli raccolti nel Paraguay, nel Matto Grosso, nel Tucuman e nella Provincia di Salta. - Bollettino dei Musei di Zoologia ed Anatomia comparata della R. Università di Torino No. 208 (vol. X): 1-24.

Salvadori T., 1896: Descrizione di una nuova specie del genere Rhamphocoelus di Chiriqui. - Bollettino dei Musei di Zoologia ed Anatomia comparata della R. Università di Torino No. 249 (vol. XI): 1-2.

Salvadori T., 1897a: Viaggio del Dott. Alfredo Borelli nel Chaco boliviano e nella Repubblica Argentina. VII. Uccelli. - Bollettino dei Musei di Zoologia ed Anatomia comparata della R. Università di Torino No. 292 (vol. XII): 1-36. 
Salvadori T., 1897b: Lista di uccelli raccolti dal Dr. Muzioli nel Tigrè e donati al Museo Zoologico di Perugia. - Bollettino dei Musei di Zoologia ed Anatomia comparata della R. Università di Torino No. 287 (vol. XII): 1-4.

Salvadori T., 1899: Viaggio del Dr. A. Borelli nel Matto Grosso e nel Paraguay. I. Nuova specie del genere Pyrrhura, Bp. - Bollettino dei Musei di Zoologia ed Anatomia comparata della R. Università di Torino No. 363 (vol. XIV): 1-2.

Salvadori T., 1900: On some Additional Species of Parrots of the Genus Pyrrhura. - Ibis, $7^{\text {th }}$ ser., VI: 667-674, pl. XIV

Salvadori T., 1902: Nuova specie del genere Ammomanes. - Bollettino dei Musei di Zoologia ed Anatomia comparata della R. Università di Torino No. 425 (vol. XVII): 1-2.

Salvadori T., 1906: Spedizione al Ruwenzori di S.A.R. Luigi Amedeo di Savoia Duca degli Abruzzi. VI. Nuove specie di Uccelli. - Bollettino dei Musei di Zoologia ed Anatomia comparata della R. Università di Torino No. 542 (vol. XXI): 1-2.

Salvadori T., 1907: Collezione di Uccelli delle vicinanze del Lago Moero, nell'Africa Centrale, raccolti dal Dott. Ascenso. - Bollettino dei Musei di Zoologia ed Anatomia comparata della R. Università di Torino No. 570 (vol. XXII): 1-9.

Salvadori T., 1908a: Description af an apparently new species of Petrel (Fregetta). - Bulletin of the British Ornithologists' Club N. CXLII (vol. XXI): 78-80.

Salvadori T., 1908b: Gyps erlangeri, nov. sp. - Bollettino dei Musei di Zoologia ed Anatomia comparata della R. Università di Torino No. 576 (vol. XXIII): 1-6.

Salvadori T., 1911: Specie apparentemente nuova del Genere Thalassogeron. - Bollettino dei Musei di Zoologia ed Anatomia comparata della R. Università di Torino No. 638 (vol. XXVI): 1-2.

Salvadori T., 1913: Nuova specie del genere Dryonastes. - Annali del Museo civico di Storia naturale di Genova 46: 5-6.

Salvadori T., 1915: Notizie storiche intorno alla Collezione Ornitologica del Museo di Torino. - Memorie della R. Accademia delle Scienze di Torino, serie 2a, vol. 65 [1916]: 1-49.

Salvadori T., 1918: Nota bibliografica intorno ad E. Benvenuti. - Rivista italiana di Ornitologia 4: 28-29.

Salvadori T. \& Antinori 0., 1872: Intorno al Cypselus horus. - Atti della Reale Accademia delle Scienze di Torino 8: 94-96.

Salvadori T. \& D'Albertis L. M., 1876: Catalogo di una collezione di uccelli dell'isola Yule e della vicina costa meridionale della penisola orientale della Nuova Guinea, raccolti da L. M. D’Albertis. - Annali del Museo civico di Storia naturale di Genova 7: 797-839.

Salvadori T. \& Festa E., 1898: Viaggio del Dott. E. Festa nella Repubblica dell'Ecuador e regioni vicine. XIII. Descrizione di tre nuove specie di uccelli. - Bollettino dei Musei di Zoologia ed Anatomia comparata della R. Università di Torino No. 330 (vol. XIII): 1-2.

Salvadori T. \& Festa E., 1899a - Viaggio del Dr. Enrico Festa nell'Ecuador. XX. Uccelli. Parte seconda. Passeres oscines. - Bollettino dei Musei di Zoologia ed Anatomia comparata della R. Università di Torino No. 357 (vol. XIV, but "XV" on first page): 1-31.

Salvadori T. \& Festa E., 1899b - Viaggio del Dr. Enrico Festa nell'Ecuador. XXI. Uccelli. Parte seconda. Passeres clamatores. - Bollettino dei Musei di Zoologia ed Anatomia comparata della R. Università di Torino No. 362 (vol. XIV, but "XV" on first page): 1-34.

Salvadori T. \& Festa E., 1900 - Viaggio del Dr. Enrico Festa nell'Ecuador. XXII. Uccelli. Parte terza. Trochili - Tinami. - Bollettino dei Musei di Zoologia ed Anatomia comparata della R. Università di Torino No. 368 (vol. XV): 1-54.

Salvadori T. \& Festa E., 1910: Nuova specie del genere Thinocorus. - Bollettino dei Musei di Zoologia ed Anatomia comparata della R. Università di Torino No. 631 (vol. XXV): 1-2.

Salvadori T. \& Festa E., 1913: Escursioni zoologiche del Dr. Enrico Festa nell'Isola di Rodi. II. Uccelli. - Bollettino dei Musei di Zoologia ed Anatomia comparata della R. Università di Torino No. 673 (vol. XXVIII): 1-24. 
Salvadori T. \& Festa E., 1914: Nuova Specie di Frosone della Sardegna. - Bollettino dei Musei di Zoologia ed Anatomia comparata della R. Università di Torino No. 681 (vol. XXIX): 1-2.

Salvadori T. \& Festa E., 1916: Alcuni uccelli della Cirenaica, colla descrizione di una nuova specie del genere Caccabis. - Bollettino dei Musei di Zoologia ed Anatomia comparata della R. Università di Torino No. 714 (vol. XXXI): 1-2.

Salvadori T. \& Giglioli E.H., 1885a: Due nuove specie di uccelli della Cocincina raccolte durante il viaggio della R. Pirofregata Magenta. - Atti della Reale Accademia delle Scienze di Torino 20: 343-345.

Salvadori T. \& Giglioli E.H., 1885b: Due nuove specie di picchi raccolte durante il viaggio intorno al mondo della Pirofregata Magenta. - Atti della Reale Accademia delle Scienze di Torino 20: 632-634.

Salvin 0., 1875: Additional Notes on the Birds of the Islands of Masafuera and Juan Fernandez. - Ibis, third series, Vol. V: 370-377.

Salvin 0., 1896: Catalogue of the Gaviae and Tubinares in the collection of the British Museum. - London: The Trustees of the British Museum, 475 pp. + VIII plates.

Sanchez Osés C.S., 2010: Vogeltypen der ornithologischen Sammlung in Übersee-Museum Bremen.pp. 21-72. - In: Becker P.-R. (ed.): Unbestimmtes erforschen. - Bramsche: Rasch Druckerei und Verlag GmbH \& Co. KG, 160 pp.

Sapeto G., 1857: Viaggio e Missione cattolica fra i Mensa i Bogos e gli Habab, con un cenno geografico e storico dell'Abissinia. - Roma: Congregazione di Propaganda Fide, 528 pp.

Savi P., 1824: Sopra una nuova specie di Sylvia. - Nuovo Giornale de' Letterati, tomo VII, N. 14: 341-344.

Savi P., 1829: Ornitologia toscana. Tomo II. - Pisa: Tip. Nistri, 302 pp.

Savi P., 1831: Ornitologia toscana. Tomo III. - Pisa: Tip. Nistri, 296 pp.

Savi P., 1831b: Sopra due specie di Motacillæ non per anche state trovate in Italia, una delle quali inedita. - Nuovo Giornale de' Letterati, N. 57, Tomo XXII, Parte Scientifica, p. 186-198.

Schifter H., Bauernfeind E. \& Schifter Th., 2007: Die Typen der Vogelsammlung des Naturhistorischen Museums Wien. Teil I. Nonpasseres. - Kataloge der wissenschaftlichen Sammlungen des Naturhistorischen Museums in Wien. Band 20, Aves, Heft 1. - Wien, Nat. Museum, $376 \mathrm{pp}$.

Sclater P.L., 1857: Catalogue of the Birds collected by M. Auguste Sallé in Southern Mexico, with Descriptions of New Species. - Proceedings of the Zoological Society of London, Part XXIV [1856]: 283-311.

Sclater P.L., 1858: On a Collection of Birds made by Signor Matteo Botteri in the vicinity of Orizaba in Southern Mexico. - Proceedings of the Zoological Society of London, Part XXV [1857]: 226-230.

Sclater P.L., 1860a. On a series of Birds collected in the vicinity of Jalapa, in Southern Mexico. - Proceedings of the Zoological Society of London, Part XXVII [1859]: 362-369.

Sclater P.L., 1860b: List of Birds collected by M. A. Boucard in the State of Oaxaca in SouthWestern Mexico, with descriptions of new species. - Proceedings of the Zoological Society of London, Part XXVII [1859]: 369-393.

Sclater P.L., 1873a: Mr. Sclater exhibited a series of new and rare birds [...]. - Proceedings of the Zoological Society of London for the Year 1873: 557-560.

Sclater P.L., 1873b: The new Bird of Paradise. - Nature 8 (August, 14): 305-306.

Sclater P.L., 1874: Characters of new species of Birds discovered in New Guinea by Signor d'Albertis. - Proceedings of the Zoological Society of London for the Year 1873: 690-698.

Sclater P.L., 1880: Note on the Rallus sulcirostris of Wallace and its Allies. - Ibis, fourth series, Vol. IV: 309-312, Plate VI.

Sclater P.L., 1890: Catalogue of the Birds in the British Museum. Vol. XV (Tracheophonae). London: The Trustees, $371 \mathrm{pp} .+\mathrm{XX}$ pl. 
Sclater P.L. \& Salvin O., 1868a: On Peruvian Birds collected by Mr. H. Whitely. Part II. Proceedings of the Zoological Society of London for the Year 1868: 173-178.

Sclater P.L. \& Salvin 0., 1868b: Synopsis of the American Rails (Rallidae). - Proceedings of the Zoological Society of London for the Year 1868: 442-470.

Seebohm H., 1881: Catalogue of the Birds in the British Museum. Volume V (Cichlomorphae: part II). - London: The Trustees, 426 pp. + XVIII Pl.

Sharpe R.B., 1877: The Birds of South Africa by Edgar Leopold Layard, new ed., Part. 4 (ca. 230 ? -336). - London: Bernard Quaritch.

Sharpe R.B., 1890: Catalogue of the Birds in the British Museum. Vol. XIII (Sturniformes). London: The Trustees, $701 \mathrm{pp} .+\mathrm{XV}$ pl.

Sharpe R.B., 1894: Catalogue of the Birds in the British Museum. Vol. XXIII (Fulicariae and Alectorides). - London: The Trustees, 353 pp. + IX pl.

Sharpe R.B., 1903: A Hand-list of the Genera and Species of Birds. Vol. IV. - London: the Trustees.

Sharpe R.B., 1908: "The specimen sent by Count Salvadori [...]". - Bulletin of the British Ornithologists' Club 21, No. 142: 80.

Shelley G.E., 1876: A Monograph of The Nectariniidae, Part II. - London: by the Author.

Shelley G.E., 1882: On some new Species of Birds from South Africa. - Proceedings of the Zoological Society of London for the Year 1882: 336-337.

Sherborn C.D., 1926a: Index animalium, sive index nominum [...]. Sectio secunda. Part IX. pp. 2009-2248. - London: by The Trustees of the British Museum.

Sherborn C.D., 1926b: Index animalium, sive index nominum [...]. Sectio secunda. Part XI. pp. 2569-2880. - London: by The Trustees of the British Museum.

Steinbacher F., 1930: Bemerkungen zur Systematik der Rohrammern, Emberiza schoeniclus (L.). - Journal für Ornithologie 78, 4: 471-487.

Steinbacher J., 1949: Typen und Typoide des Natur-Museums Senckenberg. 3. Kritisches Verzeichnis von Eduard Rüppell's und Ph. J.Cretzschmar's Vogel-Typen. - Senckenbergiana 30: 99-124.

Steinheimer F.D., 2005a: Eduard Rüppell's avian types at the Natural History Museum, Tring (Aves). - Senckenbergiana biologica 85, 2: 1-32.

Steinheimer F.D., 2005b: The nomenclature of Eduard Rüppell's birds from north-east Africa. - Bulletin of the British Ornithologists' Club 125, 3: 164-211.

Stone W. \& Mathews G.M., 1913: A list of the species of Australian Birds described by John Gould, with the location of the type-specimens. - The Austral avian record 1, 6-7:129-180.

Swinhoe R., 1860: The Ornithology of Amoy (China). - Ibis 2, 5: 45-68.

Swinhoe R., 1861: Notes on Ornithology taken between Takoo and Peking, in the neighborhood of the Peiho River, Province of Chelee, north China, from August to December 1860. - Ibis 3, n. XII: 323-345.

Swinhoe R., 1863: The Ornithology of Formosa, or Taiwan. - Ibis 5, n. XIX: 250-311.

Swinhoe R., 1868: Ornithological notes from Amoy. - Ibis, n.s., 4 (n. XIII): 52-65.

Swinhoe R., 1870: On the Plovers of the Genus Agialites found in China. - Proceedings of the Zoological Society of London for the Year 1870, Part I: 136-142.

Temminck C.-J., 1809: Histoire naturelle générale des Pigeons. - Livraison 9, p. 86, pl. 40.

Temminck C.-J., 1815: Manuel d'Ornithologie, ou tableau systématique des oiseaux qui se trouvent en Europe. - Amsterdam : J.C. Sepp \& fils, 618 pp.

Temminck C.-J., 1820: Manuel d'Ornithologie, ou tableau systématique des oiseaux qui se trouvent en Europe. Seconde édition, Première partie. - Paris: G. Dufour, 439 pp.

Temminck C.-J., 1840: Manuel d'Ornithologie, Seconde édition, Quatrième partie. - Paris : E. D’Ocagne, $691 \mathrm{pp}$.

Temminck C.-J. \& Laugier M., 1821a: Nouveau recueil de Planches Coloriées d'oiseaux, pour servir de suite et de complément aux Planches Enluminées de Buffon. - Livraison 14, Planche 84, fig. 2. 
Temminck C.-J. \& Laugier M., 1821b: Nouveau recueil de Planches Coloriées d'oiseaux, pour servir de suite et de complément aux Planches Enluminées de Buffon. - Livraison 15, Planche 89, fig. 1-2.

Temminck C.-J. \& Laugier M., 1822a: Nouveau recueil de Planches Coloriées d'oiseaux, pour servir de suite et de complément aux Planches Enluminées de Buffon. - Livraison 20, Planche 119.

Temminck C.-J. \& Laugier M., 1822b: Nouveau recueil de Planches Coloriées d'oiseaux, pour servir de suite et de complément aux Planches Enluminées de Buffon. - Livraison 27, Planche 160.

Temminck C.-J. \& Laugier M., 1822c: Nouveau recueil de Planches Coloriées d'oiseaux, pour servir de suite et de complément aux Planches Enluminées de Buffon. - Livraison 28, Planche 194.

Temminck C.-J. \& Laugier M., 1823: Nouveau recueil de Planches Coloriées d'oiseaux, pour servir de suite et de complément aux Planches Enluminées de Buffon. - Livraison 33, Planche 194.

Temminck C.-J. \& Laugier M., 1824a: Nouveau recueil de Planches Coloriées d'oiseaux, pour servir de suite et de complément aux Planches Enluminées de Buffon. - Livraison 47, Planche 281, fig. 1.

Temminck C.-J. \& Laugier M., 1824b: Nouveau recueil de Planches Coloriées d'oiseaux, pour servir de suite et de complément aux Planches Enluminées de Buffon. - Livraison 49, Planche 288.

Temminck C.-J. \& Laugier M., 1825: Nouveau recueil de Planches Coloriées d'oiseaux, pour servir de suite et de complément aux Planches Enluminées de Buffon. - Livraison 64, Planche 382, fig. 1.

Temminck C.-J. \& Laugier M., 1831a: Nouveau recueil de Planches Coloriées d'oiseaux, pour servir de suite et de complément aux Planches Enluminées de Buffon. - Livraison 88, text.

Temminck C.-J. \& Laugier M., 1831b: Nouveau recueil de Planches Coloriées d'oiseaux, pour servir de suite et de complément aux Planches Enluminées de Buffon. - Livraison 88, Planche 522.

Tortonese E., 1957: Venticinque anni di vita del Museo Zoologico di Torino (1930-1955). Natura 48: 1-27.

van den Elzen R., Dickinson E.C., Dowsett R.J. \& Harding A., 2011: Theodor von Euglin's Ornithologie Notdost-Afrika‘s, der Nilquellen- und Küsten-Gebiete des Rothen Meeres und des nördlichen Somal-Landes - Notes relevant to the dates of publication of its parts. - Vertebrate Zoology 61, 1: 161-176.

van den Hoek Ostende L.W., Dekker R.J. \& Keijl G.O., 1997: Type-specimens of birds in the National Museum of Natural History, Leiden. - Nationaal Natuurhistorisch Museum Technical Bulletin 1: 1-248.

van Oort E.D., 1910: List of a collection of Birds from Western Java and from Krakatau. - Notes from the Leyden Museum 32: 105-166.

Vieillot L.P., 1817: L'Emou noir, Dromaius ater. - In: Nouveau dictionnaire d'Histoire Naturelle, Tome X: 212-13.

Vieillot L.P., 1823: L’Aigle à queue barréee. 9. A. Fasciata. - In: Bonnaterre l'Abbé \& L. P. Vieillot (1790-1823): Tableau encycopédique et méthodique des trois règnes de la nature. II édition. Ornithologie. - Livraison 93: 1192. Paris : Panckoucke.

Violani C. \& Barbagli F., 1999: Mirafra erythrocephala Salvadori \& Giglioli, 1885, an older name for Mirafra assamica marionae Baker, 1915. - Bulletin of the British Ornithologists' Club 119 (3): 189-192.

Violani C. \& Boano G., 1990: L’Uria di Craveri Synthliboramphus craveri (Aves, Alcidae). Rivista Piemontese di Storia Naturale 11: 155-162.

Voisin C. \& Voisin J.-F., 2015: List of type specimens of birds in the collections of the Muséum national d'Histoire naturelle (Paris, France). 25. Gruiformes (Mesitornithidae, Turnicidae, 
Rallidae, Heliornithidae, Rhynochetidae and Otididae). - Journal of the National Museum (Prague), Natural History Series 184, 3: 51-73.

von Spix J.B., 1824: Avium species novae, quas in itinere per Brasiliam annis MDCCCXVIIMDCCCXX [...]. Tomus I. - Monachii: Typis Franc. Seraph. Hübschmanni, [viii] + 89 pp. + xci tab.

Walters M.P., 2003: Systematic notes on Asian birds. 39. The correct name for the Mangrove Whistler Pachycephala cinerea (Blyth). - Zoologische Verhandelingen Leiden 344: 107 - 109.

Warren R.L.M., 1966: Type-Specimens of Birds in the British Museum (Natural History). Vol. I Non-Passerines. - London: Trustees of the B.M., 320 pp.

Warren R.L.M. \& Harrison C.J.O., 1971: Type-Specimens of Birds in the British Museum (Natural History). Vol. 2 Passerines. - London, Trustees of the B.M., 628 pp.

Wetmore A., 1926: Observations on the Birds of Argentina, Paraguay, Uruguay, and Chile. - Smithsonian Institution U.S. National Museum, Bulletin 133, 448 pp.

Whitaker J.I.S., 1901: [Mr. W.R. Ogilvie Grant exhibited...] a new species of Long-tailed Titmouse. - Bulletin of the British Ornithologists' Club 11: 51-52.

Whitaker J.I.S., 1902: Further information on two recently described Species of Passerine Birds. - Ibis, Eighth series, 2: 54-58.

Wied M., 1833: Beiträge zur Naturgeschichte von Brasilien. Band IV. - Weimar: 946 pp. Witherby H.F. (ed.), 1920: A Practical Handbook of British Birds. Vol. I. - London: Witherby \& Co., $532 \mathrm{pp}$. 